\title{
Treating depression in chronically ill elderly : the evaluation of a minimal psychological intervention
}

Citation for published version (APA):

Lamers, F. (2008). Treating depression in chronically ill elderly : the evaluation of a minimal psychological intervention. [Doctoral Thesis, Maastricht University]. Datawyse / Universitaire Pers Maastricht. https://doi.org/10.26481/dis.20080926fl

Document status and date:

Published: 01/01/2008

DOI:

10.26481/dis.20080926fl

Document Version:

Publisher's PDF, also known as Version of record

\section{Please check the document version of this publication:}

- A submitted manuscript is the version of the article upon submission and before peer-review. There can be important differences between the submitted version and the official published version of record.

People interested in the research are advised to contact the author for the final version of the publication, or visit the DOI to the publisher's website.

- The final author version and the galley proof are versions of the publication after peer review.

- The final published version features the final layout of the paper including the volume, issue and page numbers.

Link to publication

\footnotetext{
General rights rights.

- You may freely distribute the URL identifying the publication in the public portal. please follow below link for the End User Agreement:

www.umlib.nl/taverne-license

Take down policy

If you believe that this document breaches copyright please contact us at:

repository@maastrichtuniversity.nl

providing details and we will investigate your claim.
}

Copyright and moral rights for the publications made accessible in the public portal are retained by the authors and/or other copyright owners and it is a condition of accessing publications that users recognise and abide by the legal requirements associated with these

- Users may download and print one copy of any publication from the public portal for the purpose of private study or research.

- You may not further distribute the material or use it for any profit-making activity or commercial gain

If the publication is distributed under the terms of Article $25 \mathrm{fa}$ of the Dutch Copyright Act, indicated by the "Taverne" license above, 


\section{Treating depression in chronically ill elderly}

The evaluation of a Minimal Psychological Intervention 
The studies presented in this dissertation were conducted under the auspices of the School for Public Health and Primary Care: CAPHRI at Maastricht University, The Netherlands. The School for Public Health and Primary Care: CAPHRI is part of the Netherlands School of Primary Care Research (CaRe), which has been acknowledged since 1995 by The Royal Netherlands Academy of Art en Sciences (KNAW).

ISBN: 978-90-5278-740-4

Cover design: Esther van de Beek

Cover photograph: Femke Lamers

Printed by Datawyse / Universitaire Pers Maastricht

(C) Copyright, F. Lamers, Maastricht 2008

All rights reserved. No parts of this thesis may be reproduced, stored in a retrieval system, or transmitted, in any form or by any means, electronic, mechanical, photocopying, recording, or otherwise, without written permission from the author or from the publisher holding the copyright of the published article. 


\title{
Treating depression in chronically ill elderly
}

\section{The evaluation of a Minimal Psychological Intervention}

\author{
Proefschrift
}

ter verkrijging van de graad van doctor aan de Universiteit Maastricht, op gezag van de Rector Magnificus,

Prof. mr. G.P.M.F. Mols,

volgens het besluit van het College van Decanen,

in het openbaar te verdedigen

op vrijdag 26 september 2008 om 12.00 uur

door

Femke Lamers 


\section{Promotores}

Prof. dr. J.Th.M. van Eijk

Prof. dr. J.A. Knottnerus

\section{Copromotor}

Dr. H. Bosma

\section{Beoordelingscommissie}

Prof. dr. F. Buntinx (voorzitter)

Prof. dr. R. van Dyck (VUMC)

Dr. M. Huibers

Prof. dr. N.C. Schaper

Prof. dr. R. de Wit

The Netherlands Organisation for Health Research and Development (ZonMw) is gratefully acknowledged for funding the studies in this thesis (grant number 945-03-047) 


\section{Inhoudsopgave}

$\begin{array}{lll}\text { Chapter } 1 & \text { General introduction } & 7\end{array}$

Chapter 2 Effectiveness and cost-effectiveness of a minimal psychological intervention to reduce non-severe depression in chronically ill elderly patients: the design of a randomised controlled trial [ISRCTN92331982]

Chapter 3 Summed score of the Patient Health Questionnaire-9 was a reliable and valid method for depression screening in chronically ill elderly patients

Chapter 4 The effectiveness of a minimal psychological intervention in chronically ill elderly patients with depression: a randomised trial (the DELTA-study)

Chapter 5 Treating depression in diabetes patients: does a minimal psychological intervention affect diabetes-specific quality of life and glycemic control? A randomised controlled trial

Chapter 6 Improving quality of life in elderly COPD patients with minor to moderate depression: a randomised trial on the effectiveness of a minimal psychological intervention

Chapter 7 Economic evaluation of a minimal psychological intervention in chronically ill elderly patients with depression: a randomised trial (the DELTA-study)

Chapter 8 General discussion

Chapter 9 Summary

Samenvatting

Co-authors and affiliations

Dankwoord

153

About the author 


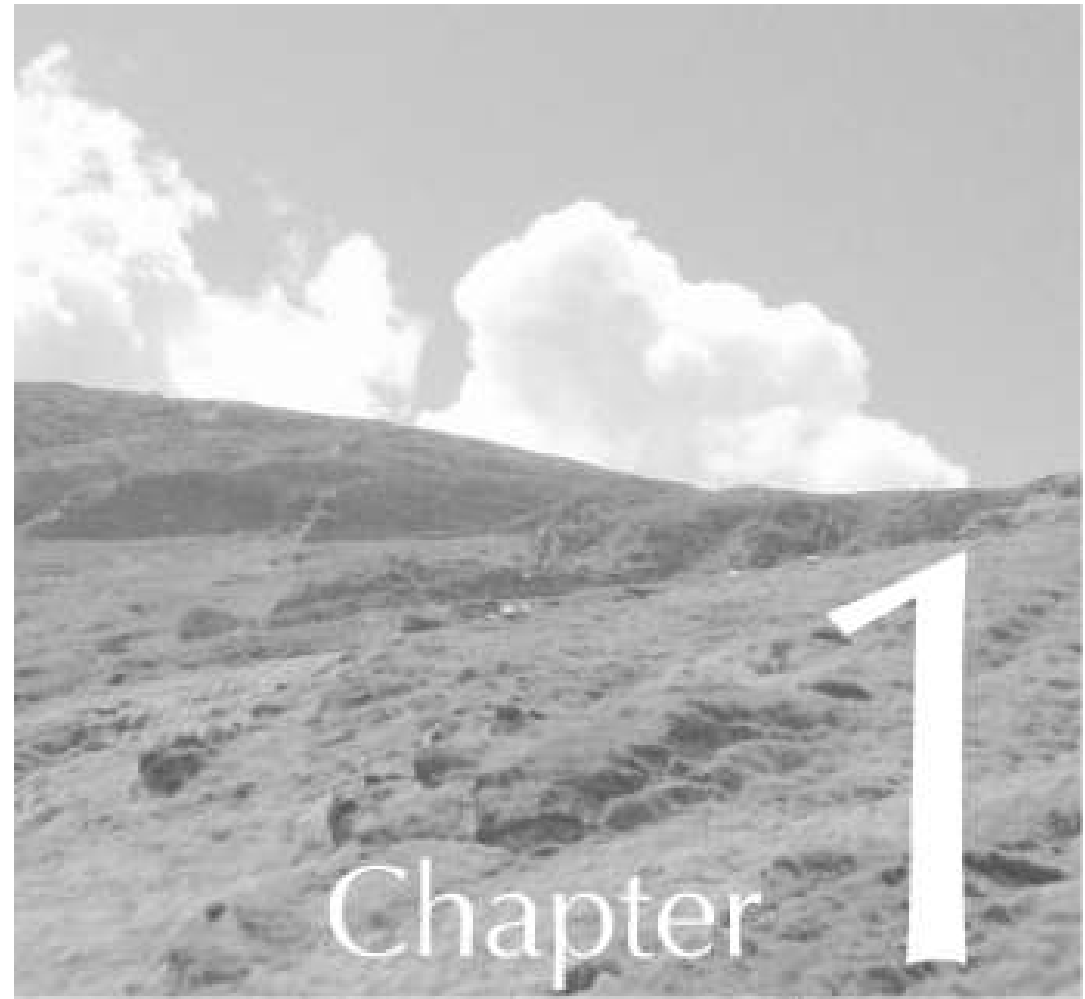

General Introduction 
With the aging of the population, ${ }^{1}$ the number of chronically ill elderly persons will increase substantially. For example, in The Netherlands, the number of persons aged 65 and over with type 2 diabetes in 2025 is estimated to have risen to 867.700 persons: a 58 percent increase compared with the situation in $2005 .{ }^{2}$ This increase of older persons with chronic somatic diseases will not only increase health care costs, but will also put further pressure on the health care system, in particular on primary care. ${ }^{3}$ In order to reduce the pressure on the health care system, keeping patients in the best possible health status is important. Reducing disabilities and thus maintaining the highest possible quality of life are therefore important targets for patients with chronic diseases, as well as for health care providers. When the chronic disease leads to impairments and functional limitations, disabilities arise. These in turn result in difficulties in doing certain activities. This whole process is known as the disablement process. ${ }^{4}$ An existing disability may reinforce itself and eventually lead to a downward spiral. Factors like medical care and self-management skills or effective coping strategies may prevent the development of further functional limitations and disability. There are also factors that may accelerate the disablement process; depression is one of them. ${ }^{5}$

\section{DEPRESSION AND ITS CONSEQUENCES}

Patients with a chronic somatic disease often face a major or minor depression. A major depression is characterised by the presence of at least five symptoms of depression during the same two-week period with at least one of the symptoms being depressed mood or loss of interest or pleasure (Table 1). The mental status should also cause significant distress or impairment in social, occupational, or other important areas of functioning. Minor depression is a state in which two to four symptoms are present, one of them being depressed mood or loss of interest or pleasure. To date, minor depression is not an official separate DSM-IV diagnosis, but is only described in research criteria. ${ }^{6}$ The prevalence of depression in elderly is estimated to be $2 \%$ for major depression and $13 \%$ for minor depression. ${ }^{7}$ Further, the risk of depression is higher in persons with chronic somatic disease. ${ }^{8}$ For instance, in chronic obstructive pulmonary disease (COPD), overall prevalence rates range from 6 to $42 \%$ for major depression and have been reported, and more than half of the COPD patients aged 65 or over reported high levels of depressive symptoms. ${ }^{9-11}$ In elderly diabetic patients, prevalence rates in elderly of up to $31 \%$ have been reported. $^{12}$

Major depression is associated with a higher mortality, ${ }^{13}$ with increased physical disability, ${ }^{14}$ and with lower quality of life. ${ }^{15}$ Furthermore, major depression is associated with higher health care utilisation and higher medical costs and is one of the most costly diseases in today's society. ${ }^{1617}$ Depression further has a negative impact on adherence to treatment and is projected to be second in rank in the worldwide leading causes of disability in $2020 .{ }^{18} 19$ Even though minor depression is not recog- 
nised as a separate diagnosis and is not included in clinical guidelines, it is also known to have detrimental effects on mortality ${ }^{13}$ and quality of life. ${ }^{15}$ Patients with minor depression have a higher risk of developing a major depression ${ }^{2021}$ and the likelihood of spontaneous remission in primary care is low (9 to $13 \%$ ). ${ }^{22}$ Finally, the economic costs of minor depression are considerable and approach the costs of major depression. $^{23}$

Table 1 Symptoms of depression (DSM-IV)

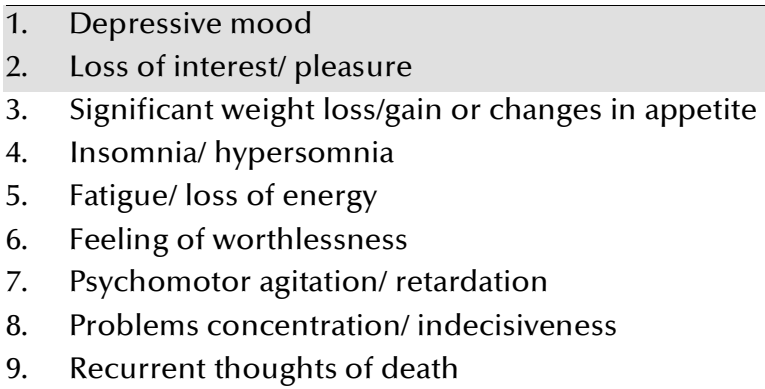

In persons with a chronic disease, a co-occurring depression, whether major or minor, may pose a serious threat to a patients' health status, as the chronic disease and depression may mutually reinforce each other. Patients may get depressed, when dealing with the consequences (disabilities) of their disease. In turn, the depression can make them less adherent to treatment regimens which may further affect their health status, leading to more complications that, in turn, bring more functional limitations and disabilities etc. ${ }^{418}$ In other words, the disablement process is reinforced. As patients with a chronic disease and co-occurring depression thus potentially run the risk of sliding into a downward spiral, action is needed to break out of this spiral.

\section{RECOGNITION OF DEPRESSION}

A problem in dealing with depression in this population is that depression in chronically ill elderly patients is often not detected or treated. ${ }^{8}$ There are several explanations for this phenomenon. Patients mainly go to their general practitioner (GP) for their somatic disease and depression has to - often unsuccessfully - 'compete' with somatic diseases for attention. ${ }^{24}$ Furthermore, somatic symptoms of depression may overlap with symptoms of the chronic somatic disease, making it more difficult to recognise the depression, as symptoms may be wrongly ascribed to the somatic disease. Moreover, because of the still existing stigma on mental health problems in elderly, older patients often do not express their depressive symptoms, because they perceive them as signs of weakness. And both GPs and patients sometimes feel that depression is a natural consequence of aging and illness and therefore does not require treatment. ${ }^{25}{ }^{26}$ This attitude towards depression in old age is worrisome, given 
the consequences of depression in chronically ill elderly patients, but especially since effective treatments for depression are available.

\section{DEPRESSION TREATMENT IN PRIMARY CARE}

Clinical guidelines for the treatment of depression in primary care recommend the use of antidepressants, psychological treatments, or combinations of the two. ${ }^{27}{ }^{28}$ The choice of treatment is based on depression severity, experienced burden, and patient preference. As minor depression is not regarded a DSM-IV diagnosis, clinical guidelines do not recommend active treatment with antidepressants or psychological treatments for minor depression, but often recommend a watchful-waiting strategy. ${ }^{27-30}$ Antidepressants are increasingly prescribed to elderly patients. ${ }^{31}{ }^{32}$ The mechanism underlying antidepressants is that they increase the level of neurotransmitters (such as serotonin and norepinephrine) in the brain by preventing their reuptake. Effects on mood become visible after several weeks. ${ }^{33}$ Antidepressants, like tricyclic antidepressants (TCAs) and the newer selective serotonine reuptake inhibitors (SSRIs), are equally effective in elderly patients, although it seems that TCAs have higher withdrawal rates, due to side effects. ${ }^{34}$

Psychological treatments include cognitive behavioural therapy (CBT) and selfmanagement approaches. Cognitive behavioural therapy (CBT) refers to therapies that aim to reduce dysfunctional emotions or behaviours by changing cognitions and/or behaviours. ${ }^{35}$ Depression, for example, is characterised by negative emotions and cognitions. These negative cognitions reinforce unhelpful behaviours (for example avoidance of certain situations), which in turn reinforce the negative cognitions. In CBT, links between dysfunctional cognitions and behaviours are identified and challenged. Through the use of new skills, such as problem solving, the patient explores alternatives for these cognitions and tries out new behaviour to test the accuracy of their cognitions and of alternatives. Repeated application of these newly acquired skills will lead to changes in behaviours and subsequently to changes in cognitions (reattribution). Once patients have broken with old cognitions and behaviours and have adopted new ones, depressive symptoms may reduce and patients are better armed against future episodes of distress and depression. Selfmanagement has many definitions and forms; Self-management for chronic conditions often refers to methods, skills, and strategies by which patients can effectively manage (the consequences of) their disease in terms of daily functioning, and shares much of its theoretical background with $\mathrm{CBT} .{ }^{3637}$ Active self-managers take responsibility for their own health status and are active participants in their own treatment. Three self-management tasks can be distinguished: 1) medical management, like adhering to medication and lifestyle rules, 2) role management, that involves maintaining, changing and or developing new meaningful life roles, and 3) emotional management, referring to the way a person deals with the emotional consequences of 
a disease. Skills to successful self-management include problem-solving, decision making, action planning, and self-tailoring. ${ }^{38}$

\section{DEPRESSION TREATMENT IN CHRONICALLY ILL ELDERLY PATIENTS}

Although the effectiveness of antidepressants has been shown in elderly patients, ${ }^{34} 39$ antidepressant treatment in chronically ill elderly persons may nevertheless encounter problems, like non-adherence, side-effects, or problems arising from polypharmacy. ${ }^{40-42}$ Psychological treatments, on the other hand, do not have these problems. Psychological treatments have shown to be effective in reducing minor and major depression in elderly persons. ${ }^{43-45}$ The presence of a chronic somatic disease might complicate an effective treatment of depression. Several studies showed, however, that these psychological treatments work just as well in elderly with chronic somatic diseases. ${ }^{46-53}$ CBT appears as effective as antidepressants ${ }^{54}$ and has been shown to be more enduring than antidepressants, ${ }^{55}$ as patients learn skills that they continue to use throughout their lives. For chronically ill patients, having proper skills to deal with the consequences of their disease is important. Therefore, in chronically ill elderly persons with depression, psychological treatments providing these enduring skills may be preferable over antidepressants. These (short-term) psychological interventions also seem appropriate for chronically ill elderly with minor depression. Although watchful waiting is currently advised for minor depression, the intervention may nevertheless be helpful in minimizing depressive symptoms and reducing the risk of developing major depression in chronically ill persons. But above all, the skills they learn may also be of great benefit to them in the long term, when they face the consequences of their progressing chronic disease.

Psychological treatments for depression are often not available within primary care settings. GPs may lack the necessary training or they do not have the time to administer a psychological intervention. However, studies show that nurses can be successfully trained to administer psychological interventions, like CBT, to depressed patients. ${ }^{5657}$ As primary care nurses in many countries are already involved in disease management programs for chronically ill patients and see patients on a regular basis, they are good candidates for the task of depression detection and for administering psychological interventions. In doing so, they can complement the care of the GP and may also reduce the burden on the GP.

\section{THE DELTA STUDY}

This thesis describes from the results of the DELTA study (Depression in Elderly with Long-Term Afflictions). The DELTA-study is a randomised controlled trial comparing a nurse-led led Minimal Psychological Intervention (MPI) with care as usual in 361 elderly primary care patients. The MPI is based on CBT and self-management ap- 
proaches (role and emotional management) and is tailored to the patients' needs. This skill-based intervention aims to reduce depressive symptoms through improving coping-skills and enhancing self-efficacy and mastery. Trained nurses administer the intervention. Participants had either diabetes mellitus type II (DM) or chronic obstructive pulmonary disease (COPD), and had a co-morbid depression. Patients were included in the DELTA-study if they had co-occuring minor depression, or mild or moderate major depression. Patients with severe major depression were excluded as the intervention was considered too short and minimal (1 to 10 sessions) for these cases. ${ }^{27}$ Patients were followed up to a year after inclusion.

\section{AIMS AND OBJECTIVES}

Main objective of this study was to evaluate both the effectiveness and costeffectiveness of the nurse-led Minimal Psychological Intervention (MPI) in reducing depressive symptoms and improving quality of life in elderly diabetic and COPD patients with a co-occurring minor to moderate depression.

A second aim was to evaluate whether the effect of the MPI was generic across DM and COPD patients. Although chronic somatic diseases differ from each other in nature, symptoms, course, and consequences for daily life, patients with different chronic diseases probably face similar adaptive tasks and challenges in coping with their disease. ${ }^{3858}$ Therefore, it was expected that the effect of an intervention aimed at reducing depression is generic across different diseases.

A third aim was to evaluate relevant disease-specific outcomes for COPD patients and diabetic patients separately.

A final objective addressed in this thesis was to validate the Patient Health Questionnaire-9 (PHQ-9) that was used for depression screening. Although this questionnaire has been validated in different populations, the recognition of depression in chronically ill elderly persons may be problematic given the potential overlapping of depressive symptoms with symptoms of chronic somatic disease, misconceptions of elderly persons about depression and the existing stigma on mental health problems in elderly, making persons reluctant to discuss their depressed mood.

\section{OUTLINE OF THIS THESIS}

Chapter 2 of this thesis addresses the study design and rationale of the DELTA-study. Chapter 3 reports on the reliability and validity of the screening instrument used in the DELTA-study, the Patient Health Questionnaire-9 (PHQ-9). For this purpose, additional data from a group of non-depressed patients were collected. In Chapter 4, the results on the effectiveness of the MPI on depressive symptoms and quality of life are presented. It is also examined whether or not the intervention is generic across DM and COPD patients. Chapter 5 and 6 report on the effects of the MPI on disease- 
specific outcomes for DM and COPD patients. In Chapter 5, the effects of the MPI on diabetes symptoms, diabetes-related emotional distress, and HbA1c-levels, an indicator of glycemic control, are described. Chapter 6 addresses the COPD patients, in whom depression and anxiety often co-occur. Both depressive symptoms and symptoms of anxiety, as well as COPD-specific quality of life were used as outcome measures. Chapter 7 reports on the cost-effectiveness of the MPI from a societal perspective. For this purpose, all relevant health care related costs as well as production losses were collected. In Chapter 8 , the results of the DELTA-study are discussed, including the limitations of the study and generalisability of the results. Recommendations for future developments and research are also discussed. 


\section{CHAPTER 1}

\section{REFERENCES}

1. World Health Organization. Active Ageing: A Policy Framework. Geneva: World Health Organization, 2002.

2. Blokstra A, Baan CA, Boshuizen HC, Feenstra TL, Hoogenveen RT, Picavet HSJ, et al. Vergrijzing en toekomstige ziektelast; Prognose chronische ziekteprevalentie 2005-2025 [Impact of the ageing population on burden of disease. Projections of chronic disease prevalence for 2005-2025]. In: Blokstra A, Verschuren WMM, editors. Bilthoven: RIVM, 2007.

3. De Bakker DH, Polder JJ, Sluijs EM, Treurniet HF, Hoeymans N, Hingstman L, et al. Op één lijn; Toekomstverkenning eerstelijnszorg 2020. Bilthoven: RIVM, 2005.

4. Verbrugge LM, Jette AM. The disablement process. Soc Sci Med 1994;38(1):1-14.

5. van Gool CH, Kempen GI, Penninx BW, Deeg DJ, Beekman AT, van Eijk JT. Impact of depression on disablement in late middle aged and older persons: results from the Longitudinal Aging Study Amsterdam. Soc Sci Med 2005;60(1):25-36.

6. American Psychiatric Association. DSM-IV-TR: Diagnostic and statistical manual of mental disorders. fourth edition, text revision ed. Washington DC: American Psychiatric Association, 2000.

7. Beekman AT, Deeg DJ, van Tilburg T, Smit JH, Hooijer C, van Tilburg W. Major and minor depression in later life: a study of prevalence and risk factors. J Affect Disord 1995;36(1-2):65-75.

8. Djernes JK. Prevalence and predictors of depression in populations of elderly: a review. Acta Psychiatr Scand 2006;113(5):372-87.

9. Kunik ME, Roundy K, Veazey C, Souchek J, Richardson P, Wray NP, et al. Surprisingly high prevalence of anxiety and depression in chronic breathing disorders. Chest 2005;127(4):1205-11.

10. Mikkelsen RL, Middelboe T, Pisinger C, Stage KB. Anxiety and depression in patients with chronic obstructive pulmonary disease (COPD). A review. Nord J Psychiatry 2004;58(1):65-70.

11. Coultas DB, Edwards DW, Barnett B, Wludyka P. Predictors of depressive symptoms in patients with COPD and health impact. Copd 2007;4(1):23-8.

12. Ali S, Stone MA, Peters JL, Davies MJ, Khunti K. The prevalence of co-morbid depression in adults with Type 2 diabetes: a systematic review and meta-analysis. Diabet Med 2006;23(11):1165-73.

13. Penninx BW, Geerlings SW, Deeg DJ, van Eijk JT, van Tilburg W, Beekman AT. Minor and major depression and the risk of death in older persons. Arch Gen Psychiatry 1999;56(10):889-95.

14. Penninx BW, Deeg DJ, van Eijk JT, Beekman AT, Guralnik JM. Changes in depression and physical decline in older adults: a longitudinal perspective. J Affect Disord 2000;61(1-2):1-12.

15. Spitzer RL, Kroenke K, Linzer M, Hahn SR, Williams JB, deGruy FV, 3rd, et al. Health-related quality of life in primary care patients with mental disorders. Results from the PRIME-MD 1000 Study. JAMA 1995;274(19):1511-7.

16. Katon WJ, Lin E, Russo J, Unutzer J. Increased medical costs of a population-based sample of depressed elderly patients. Arch Gen Psychiatry 2003;60(9):897-903.

17. Katz IR. On the inseparability of mental and physical health in aged persons: Lessons from depression and medical comorbidity. Am J Geriatr Psychiatry 1996;4(1):1-16.

18. Katon WJ. Clinical and health services relationships between major depression, depressive symptoms, and general medical illness. Biol Psychiatry 2003;54(3):216-26.

19. Murray CJL, Lopez AD. Alternative visions of the future: projecting mortality and disability, 1990-2020. In: Murray CJL, Lopez AD, editors. The global burden of disease: a comprehensive assessment of mortality and disability from diseases, injuries, and risk factors in 1990 and projected to 2020. Cambridge, M.A.: Harvard University Press, 1996:325-395.

20. Cuijpers P, de Graaf R, van Dorsselaer S. Minor depression: risk profiles, functional disability, health care use and risk of developing major depression. J Affect Disord 2004;79(1-3):71-9.

21. Lyness JM, Heo M, Datto CJ, Ten Have TR, Katz IR, Drayer R, et al. Outcomes of minor and subsyndromal depression among elderly patients in primary care settings. Ann Intern Med 2006;144(7):496-504.

22. Hegel MT, Oxman TE, Hull JG, Swain K, Swick H. Watchful waiting for minor depression in primary care: remission rates and predictors of improvement. Gen Hosp Psychiatry 2006;28(3):205-12.

23. Cuijpers P, Smit F, Oostenbrink J, de Graaf R, Ten Have M, Beekman A. Economic costs of minor depression: a population-based study. Acta Psychiatr Scand 2007;115(3):229-36. 
24. Schwenk TL. Diagnosis of late life depression: the view from primary care. Biol Psychiatry 2002;52(3):15763.

25. Graham N, Lindesay J, Katona C, Bertolote JM, Camus V, Copeland JR, et al. Reducing stigma and discrimination against older people with mental disorders: a technical consensus statement. Int J Geriatr Psychiatry 2003;18(8):670-8.

26. Unutzer J. Diagnosis and treatment of older adults with depression in primary care. Biol Psychiatry 2002;52(3):285-92.

27. National Institute for Clinical Excellence. Depression. Management of depression in primary and secondary care. London: NHS, NICE, 2004.

28. van Marwijk HWJ, Grundmeijer HGLM, Bijl D, van Gelderen MG, de Haan M, van Weel-Baumgarten EM, et al. NHG-standaard Depressieve stoornis (depressie) (eerste herziening). Huisarts Wet 2003;46(11):61423.

29. Whooley MA, Simon GE. Managing depression in medical outpatients. N Engl J Med 2000;343(26):1942-50.

30. Williams JW, Jr., Barrett J, Oxman T, Frank E, Katon W, Sullivan M, et al. Treatment of dysthymia and minor depression in primary care: A randomized controlled trial in older adults. JAMA 2000;284(12):1519-26.

31. Hansen DG, Rosholm JU, Gichangi A, Vach W. Increased use of antidepressants at the end of life: population-based study among people aged 65 years and above. Age Ageing 2007;36(4):449-54.

32. Mamdani MM, Parikh SV, Austin PC, Upshur RE. Use of antidepressants among elderly subjects: trends and contributing factors. Am J Psychiatry 2000;157(3):360-7.

33. CVZ CFH. Farmacotherapeutisch Kompas (Pharmacotherapeutic Compass). 21 ed. Amstelveen: College voor zorgverzekeringen, 2005.

34. Mottram P, Wilson K, Strobl J. Antidepressants for depressed elderly. Cochrane Database Syst Rev 2006(1):CD003491.

35. Brewin CR. Theoretical foundations of cognitive-behavior therapy for anxiety and depression. Annu Rev Psychol 1996;47:33-57.

36. Barlow J, Wright C, Sheasby J, Turner A, Hainsworth J. Self-management approaches for people with chronic conditions: a review. Patient Educ Couns 2002;48(2):177-87.

37. Newman S, Mulligan K, Steed L. What is meant by self-management and how can its efficacy be established? Rheumatology (Oxford) 2001;40(1):1-4.

38. Lorig KR, Holman H. Self-management education: history, definition, outcomes, and mechanisms. Ann Behav Med 2003;26(1):1-7.

39. Wilson K, Mottram P, Sivanranthan A, Nightingale A. Antidepressant versus placebo for depressed elderly. Cochrane Database Syst Rev 2001(2):CD000561.

40. Lebowitz BD, Pearson JL, Schneider LS, Reynolds CF, 3rd, Alexopoulos GS, Bruce ML, et al. Diagnosis and treatment of depression in late life. Consensus statement update. JAMA 1997;278(14):1186-90.

41. Montgomery SA. Late-life depression: rationalizing pharmacological treatment options. Gerontology 2002;48(6):392-400.

42. Spina E, Scordo MG. Clinically significant drug interactions with antidepressants in the elderly. Drugs Aging 2002;19(4):299-320.

43. Cuijpers P, van Straten A, Smit F. Psychological treatment of late-life depression: a meta-analysis of randomized controlled trials. Int. J. Geriatr. Psychiatry 2006;21(12):1139-49.

44. McCusker J, Cole M, Keller E, Bellavance F, Berard A. Effectiveness of treatments of depression in older ambulatory patients. Arch. Intern. Med. 1998;158(7):705-12.

45. Pinquart M, Duberstein PR, Lyness JM. Treatments for later-life depressive conditions: a meta-analytic comparison of pharmacotherapy and psychotherapy. Am. J. Psychiatry 2006;163(9):1493-501.

46. Bogner HR, Cary MS, Bruce ML, Reynolds CF, 3rd, Mulsant B, Ten Have T, et al. The role of medical comorbidity in outcome of major depression in primary care: the PROSPECT study. Am J Geriatr Psychiatry 2005;13(10):861-8.

47. Brody BL, Roch-Levecq AC, Kaplan RM, Moutier CY, Brown SI. Age-related macular degeneration: selfmanagement and reduction of depressive symptoms in a randomized, controlled study. J Am Geriatr Soc 2006;54(10):1557-62.

48. Brody BL, Roch-Levecq AC, Thomas RG, Kaplan RM, Brown SI. Self-management of age-related macular degeneration at the 6-month follow-up: a randomized controlled trial. Arch Ophthalmol 2005;123(1):46-53. 


\section{CHAPTER 1}

49. Ciechanowski P, Wagner E, Schmaling K, Schwartz S, Williams B, Diehr P, et al. Community-integrated home-based depression treatment in older adults: a randomized controlled trial. JAMA 2004;291(13):156977.

50. Emery CF, Schein RL, Hauck ER, Maclntyre NR. Psychological and cognitive outcomes of a randomized trial of exercise among patients with chronic obstructive pulmonary disease. Health Psychol. 1998;17(3):232-40.

51. Harpole LH, Williams JW, Jr., Olsen MK, Stechuchak KM, Oddone E, Callahan CM, et al. Improving depression outcomes in older adults with comorbid medical illness. Gen Hosp Psychiatry 2005;27(1):4-12.

52. Kunik ME, Braun U, Stanley MA, Wristers K, Molinari V, Stoebner D, et al. One session cognitive behavioural therapy for elderly patients with chronic obstructive pulmonary disease. Psychol. Med. 2001;31(4):717-23.

53. Mossey JM, Knott KA, Higgins M, Talerico K. Effectiveness of a psychosocial intervention, interpersonal counseling, for subdysthymic depression in medically ill elderly. J. Gerontol. A. Biol. Sci. Med. Sci. 1996;51(4):M172-8.

54. DeRubeis RJ, Hollon SD, Amsterdam JD, Shelton RC, Young PR, Salomon RM, et al. Cognitive therapy vs medications in the treatment of moderate to severe depression. Arch Gen Psychiatry 2005;62(4):409-16.

55. Hollon SD, DeRubeis RJ, Shelton RC, Amsterdam JD, Salomon RM, O'Reardon JP, et al. Prevention of relapse following cognitive therapy vs medications in moderate to severe depression. Arch Gen Psychiatry 2005;62(4):417-22.

56. Hunkeler EM, Meresman JF, Hargreaves WA, Fireman B, Berman WH, Kirsch AJ, et al. Efficacy of nurse telehealth care and peer support in augmenting treatment of depression in primary care. Arch Fam Med 2000;9(8):700-8.

57. Mynors-Wallis LM, Gath DH, Day A, Baker F. Randomised controlled trial of problem solving treatment, antidepressant medication, and combined treatment for major depression in primary care. BMJ 2000;320(7226):26-30.

58. Lorig KR, Sobel DS, Stewart AL, Brown BW, Jr., Bandura A, Ritter P, et al. Evidence suggesting that a chronic disease self-management program can improve health status while reducing hospitalization: a randomized trial. Med Care 1999;37(1):5-14. 


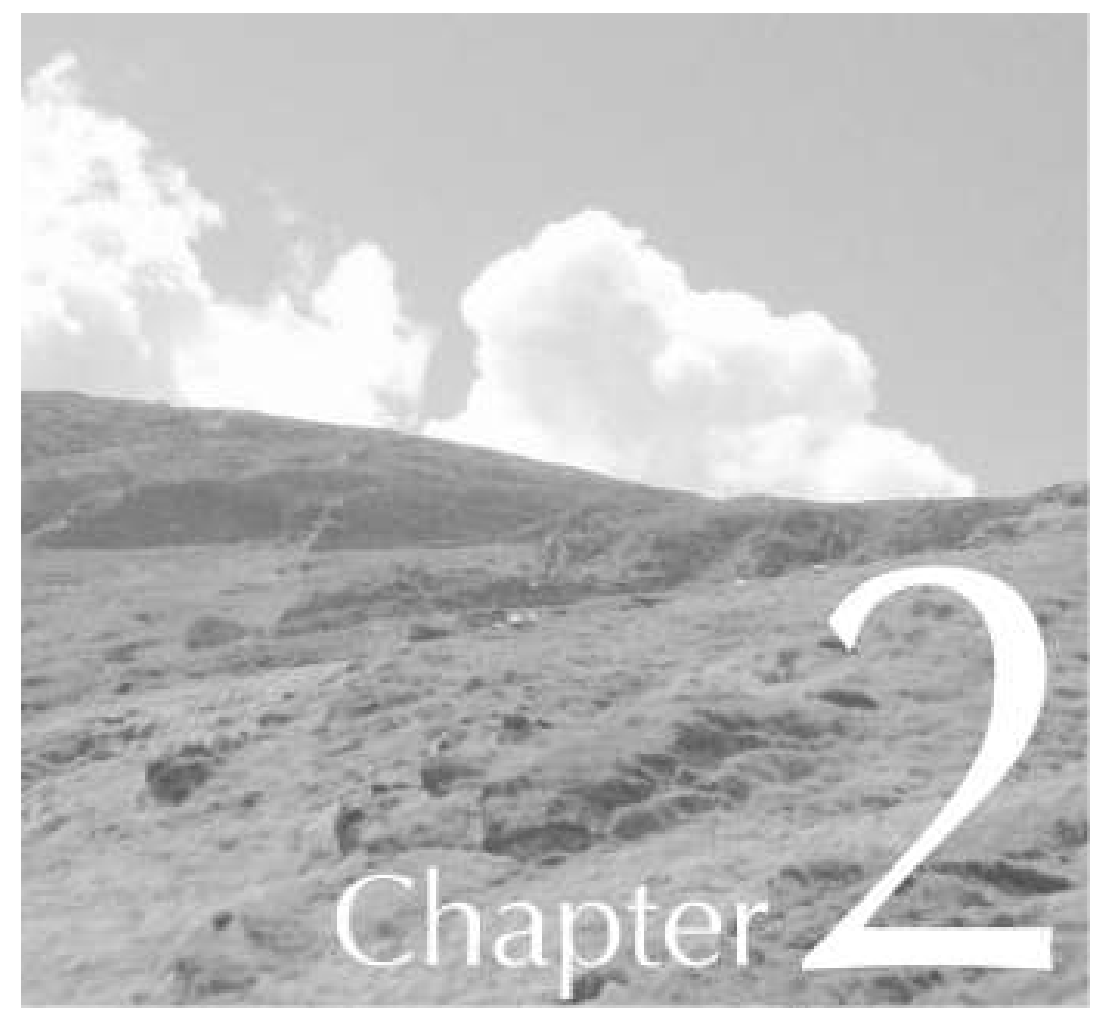

Effectiveness and cost-effectiveness of a minimal psychological intervention to reduce non-severe depression in chronically ill elderly patients: the design of a randomised controlled trial [ISRCTN92331982] 
CHAPTER 2 


\section{ABSTRACT}

Background: Depression is a prevalent disorder in chronically ill elderly persons. It may decrease quality of life, and increase functional disability, medical costs, and health care utilisation. Because patients may slip into a downward spiral, early recognition and treatment of depression is important. Depression can be treated with antidepressants or psychological interventions; the latter can also be applied by trained paraprofessionals.

In this paper, we describe the design of the DELTA study (Depression in Elderly with Long-Term Afflictions). The first objective of the DELTA study is to evaluate the effectiveness and cost-effectiveness of a minimal psychological intervention (MPI) to reduce depression in chronically ill elderly patients. The second objective is to evaluate whether a potential effect of the MPI may differ between types of chronic illnesses. The tailor-made intervention is administered by nurses, who are trained in the principles of cognitive behavioural therapy and self-management.

Methods/Design: DELTA is a two-armed randomised controlled trial, comparing MPI to usual care. A total number of 180 patients with diabetes mellitus type II (DM) and 180 patients with chronic obstructive pulmonary disease (COPD), who in addition suffer from non-severe depression, will be included in the study. In our study, nonsevere depression is defined as having minor depression, mild major depression or moderate major depression. The primary outcome measure is depression using the Beck Depression Inventory. Secondary outcome measures include quality of life, daily functioning, self-efficacy, autonomy, and participation. In the economic evaluation, cost-effectiveness and cost-utility ratios will be calculated. Furthermore, a process evaluation will be carried out.

Analyses will include both univariate and multivariate techniques and according to the intention to treat principle. The economic evaluation will be done from a societal perspective and data of the process evaluation will be analysed using descriptive techniques. 
CHAPTER 2 


\section{BACKGROUND}

Depression is a prevalent and disabling disorder, especially in patients with chronic illnesses, such as diabetes mellitus type II (DM) and chronic obstructive pulmonary disease (COPD). In older patients with DM, prevalence rates of clinical relevant depression range from 14 to $17 \% .^{12}$ In older patients with COPD, prevalence rates of $25 \%$ for minor depression have been reported. ${ }^{3}$ Prevalence rates of major depression in older COPD patients range from 6 to $42 \%{ }^{4}$

Persons suffering from minor or major depression have increased mortality risks and a decreased quality of life compared with non-depressed persons. ${ }^{5-7}$ Furthermore, depression has been shown to increase health care utilisation, ${ }^{89}$ medical costs, ${ }^{10}$ and disability. ${ }^{811}$ Since disability predicts the onset of depression and depression itself may further heighten risks of a progressing disability, this process of mutual reinforcement may lead to a downward spiral. ${ }^{11-14}$ In addition, depression impairs one's ability to adhere to disease management regimens (diet, exercise, quitting smoking, taking medication regularly), potentially worsening the course of the chronic illness. ${ }^{15}$ ${ }^{16}$ Hence, an early detection of depressive symptoms and treatment of depression is important in chronically ill elderly persons, thereby preventing or breaking a downward spiral. In primary care however, depression often remains undetected. ${ }^{17}$ General practitioners have limited time and furthermore, current Dutch guidelines for DM and COPD don't take into account the psychological consequences of the chronic illness.

Available treatment options are antidepressants or psychological interventions. The effectiveness of antidepressants has been extensively studied and proven in major depression. ${ }^{18}$ Since there is no clear evidence of the effectiveness of antidepressants in minor depression, ${ }^{19}{ }^{20}$ clinical guidelines advise against using antidepressants in minor depression. ${ }^{2122}$ Cognitive therapy (CT) seems to be as effective as antidepressants in severe depression, ${ }^{23}$ and also in patients with mild and moderate depression. ${ }^{24}$ Furthermore, CT seems to have an enduring effect. ${ }^{25}$ It is also increasingly recognised that chronically ill elderly suffering from depression might benefit from psychosocial support and improving coping skills, such as self-management techniques. ${ }^{21}{ }^{26}$ In a study with DM type II patients, cognitive behavioural therapy (CBT) in combination with supportive diabetes education proved to be an effective treatment for major depression. ${ }^{27}$ Similarly, self-management strategies in COPD patients have been reported to improve the patients' health status and to reduce hospital admissions. $^{28}$

Accumulating evidence shows that primary care staff can be trained in psychological interventions for depression. ${ }^{29}$ Several studies reported that practice nurses can successfully administer interventions to reduce depression in primary care settings. ${ }^{3031}$ We developed a minimal psychological intervention (MPI), based on principles of self-management and CBT. The intervention is administered by nurses and aims to 
reduce non-severe depression in chronically ill elderly persons. Findings of a prior smaller pilot study showed that the intervention was feasible and acceptable to patients. Furthermore, the training programme, developed to teach nurses to administer the intervention, appeared to be feasible, attractive and successful among nurses. ${ }^{32}$

In this contribution, we present the design of the DELTA study (Depression in Elderly with Long-Term Afflictions). The first objective of this randomised controlled trial (RCT) is to evaluate the effectiveness and cost-effectiveness of an MPI that is administered by a nurse and aims to reduce non-severe depression in chronically ill elderly patients. The effects of the MPI are compared with usual care. The second objective is to evaluate whether a potential effect of the MPI is different between types of chronic illnesses.

\section{DESIGN AND METHODS}

\section{Design}

The DELTA study is a two-armed randomised controlled trial, in which an effect evaluation, an economic evaluation and a process evaluation will be carried out. A total number of 360 patients will be included, 180 of which are patients with DM and 180 are patients suffering from COPD. We chose DM and COPD because first, they are highly prevalent in primary care. Second, they have a different course and prognosis. DM can be seen a gradual progressive illness, whereas COPD as a gradual relapsing condition. ${ }^{33}$ This difference enables us to test whether the intervention is potentially generic. Approval for conducting this study was granted by the Medical Ethics Committee of the Maastricht University/ Academic Hospital Maastricht.

\section{Setting and recruitment}

In general practices in the southern part of Limburg, a province in the south of the Netherlands, all patients of 60 years and over with DM and or COPD were selected by the general practitioner, the general practitioner's assistant, or the research assistant. Selection was made using ICPC codes (T90, R91.01, R95, R99.06) if possible and otherwise by medication prescriptions (those drugs which are most often prescribed by the general practitioner for these chronic illnesses). In the last phase of patient selection, the general practitioner applied the inclusion and exclusion criteria using a pre-coded form with checkboxes for each criterion (Table 1).

All selected patients received a letter from their general practitioner with a request to complete a short screening questionnaire. This questionnaire, the Patient Health Questionnaire-9 (PHQ-9), consists of nine questions regarding the prevalence of symptoms of depression over the last two weeks. The response options are: "Not at all", "Several days", "More than half the days" and "Nearly every day". Its brevity and 
the fact that it is a self-administered questionnaire make it a useful tool in screening for depression in primary care. The PHQ-9 has been validated for both diagnosing depression and measuring severity. ${ }^{34-36}$ Five questions on demographic variables were included in the questionnaire. Patients received a reminder by telephone two weeks after the questionnaire had been sent. All patients who scored at least 2 depressive symptoms at least at "more than half the days" and at least one of these symptoms was depressed mood or anhedonia, were invited to participate in an interview to confirm or reject the diagnosis of depression. The Mini International Neuropsychiatric Interview (MINI) was used to confirm the diagnosis from the PHQ-9. The interview took place at the patient's home and was administered by a trained nurse. The MINI is a validated and reliable diagnostic structured interview, covering 17 disorders based on DSM-IV criteria. ${ }^{37}$ An extra diagnosis box for minor depression was added to the MINI, based on the research criteria for minor depression as described in the DSMIV. ${ }^{39}$ Furthermore, the Hamilton Depression Rating Scale (HDRS) was used to determine the severity of the depression. ${ }^{4041}$ Patients were excluded if they met one of the following criteria: if the MINI indicated a major depression in combination with a score above 18 (indicating a severe depression) on the HDRS, if the MINI indicated suicidal risk, or if the MINI indicated no depression at all (Table 1). Patients with a major depression and/or suicidal risk were referred back to their general practitioner. All remaining eligible patients (patients with a minor depression, non-severe major depression, or dysthymia) were invited to participate in the study and to give their informed consent.

Table 1 Inclusion and exclusion criteria as applied by the general practitioner* or research nurse ${ }^{+}$

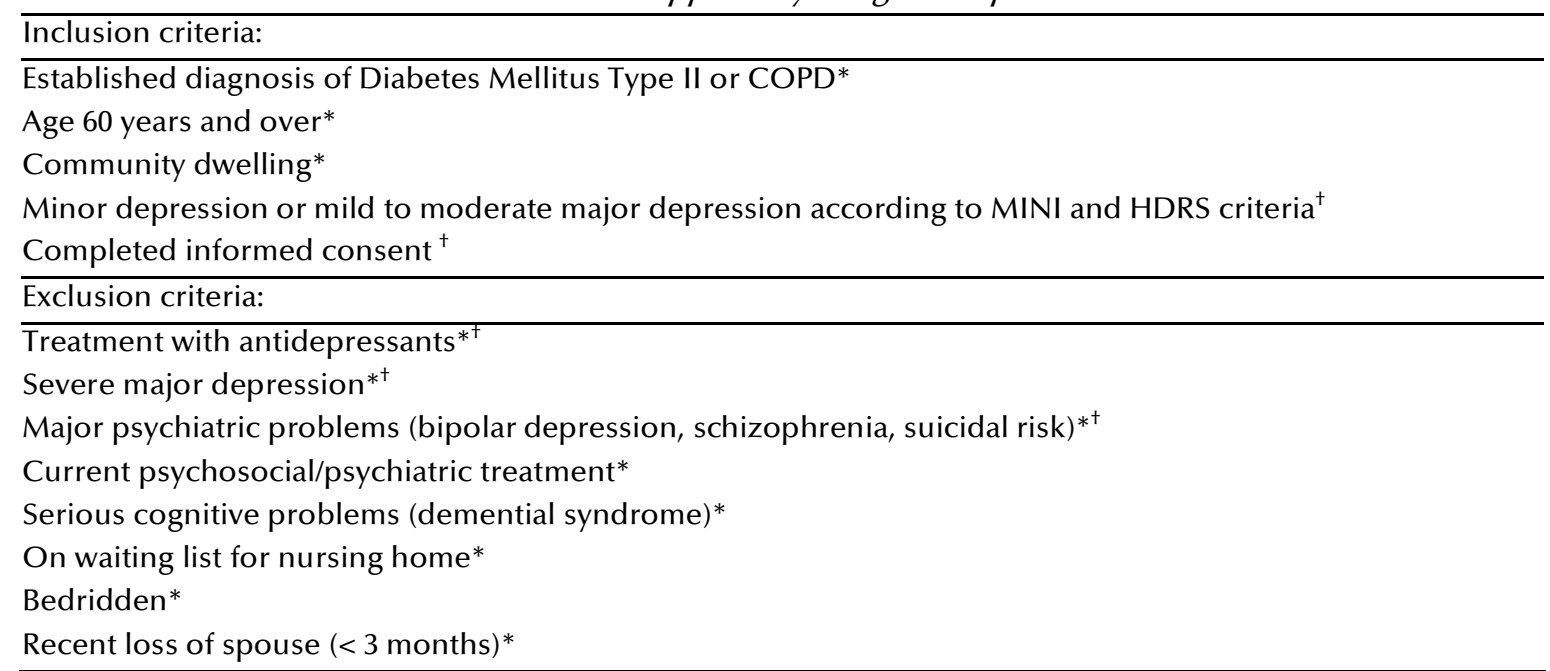

\section{Randomisation}

After having signed the informed consent form, patients enrolled in the study and filled in the baseline questionnaire. After having completed the baseline questionnaire, patients were assigned to either the intervention or control group. Randomisa- 
tion was performed by an external agency using a computerized random number generator. In order to avoid an imbalance of chronic illness and general practice (care level) over the two groups, stratification for general practice and chronic illness (DM or COPD) was performed. Furthermore, to obtain equal numbers in both arms, a blocked design with a block size of two was applied. The intervention group received a Minimal Psychological Intervention, while the control group received usual care as given by their general practitioner, according to the guidelines for the specific chronic illness.

\section{Minimal Psychological Intervention}

The intervention was given by a trained nurse, at the patient's own home. During a period of at most three months, patients received a maximum of 10 visits from the nurse. The number of visits depended on the patient's progress.

The Minimal Psychological Intervention contains elements from the Chronic Disease Self-Management Program (CDSMP) by Lorig and Gonzales, ${ }^{42}$ the Reattribution model from Goldberg ${ }^{43}$ and from the work of the project group of the Interventie Studie Eerste Lijn (INSTEL), ${ }^{44}$ as previously described. ${ }^{32}$ The intervention aims at teaching patients to take responsibility for day-to-day management of their illness and its consequences. In short, it consists of five phases:

Phase 1: The nurse explores the patient's cognitions on the origin of symptoms and complaints, and their relation to limitations and behaviour.

Phase 2: The patient keeps a diary, where he or she records symptoms, complaints, thoughts, worries, related feelings, and behaviour.

Phase 3: Using information from the diary, the nurse challenges the patient to link his or her mood and consequent behaviour to the course of the chronic illness. A distinction will be made between complaints related to the illness itself, and those related to the emotional and behavioural consequences of the illness.

Phase 4: Introduction of the self-management approach by the nurse. The patient explores his or her possibilities to alter his or her behaviour. He or she then makes a plan on how to solve perceived problems and sets specific goals to be reached before the next visit from the nurse.

Phase 5: Evaluation of the progress in achieving the goals.

After a patient has completed these five phases successfully, he or she is supposed to be able to apply the self-management approach to any situation or problem he or she may encounter in the future. In consultation with the patient, the nurse can then decide to conclude the series of intervention visits. 


\section{The training program for nurses}

\section{ADMINISTERING THE MINI}

In an $8 \mathrm{~h}$ session, the nurses were trained how to confirm a diagnosis of depression by using the MINI and HDRS by a psychiatrist.

\section{APPLYING THE MINIMAL PSYCHOLOGICAL INTERVENTION}

During three $8 \mathrm{~h}$ sessions, with 2-week intervals, four nurses were trained by two experienced trainers (a psychologist/cognitive behaviour therapist, and a general practitioner) on how to apply the intervention. In between training days, nurses practised their newly learned skills on a pilot patient. As mentioned earlier, the training program has been shown to be feasible, attractive and successful among nurses. ${ }^{32}$ Booster sessions were being held regularly during the study, and both a psychiatrist and a psychologist could be contacted by telephone to discuss cases at any time.

\section{Data collection}

Data was collected at five points in time: at baseline (T0), one week after the intervention period (T1), and at three, six and nine months after the intervention period (T2, T3, T4) (Fig. 1). The intervention period for patients allocated to the intervention group varies from one week to three months. The intervention period of the control group is fixed at six weeks, which is estimated to be the mean duration of the intervention period in the intervention group. Data were collected using self-administered questionnaires and cost diaries in combination with interviews by telephone.

\section{Effect evaluation}

Table 2 provides an overview of the measures of the effect and economic evaluation, and time of assessment.

\section{PRIMARY OUTCOME MEASURES}

The primary outcome measure in this study was level of depression, measured with the Beck Depression Inventory (BDI). ${ }^{45} 46$ The BDI consists of 21 items measuring symptoms of depression and has proven to be a valid and reliable too. ${ }^{47}$

\section{SECONDARY OUTCOME MEASURES}

Secondary outcome measures in the study were: Quality of life measured with the Short Form-36 (SF-36), ${ }^{48}$ disease-specific quality of life assessed with the Problem Areas in Diabetes questionnaire (PAID-1) for diabetes patients, ${ }^{49}$ and the St. George's Respiratory Questionnaire (SGRQ) for patients with pulmonary disease. ${ }^{50} 51$ Furthermore, daily functioning was assessed with the Activities of Daily Life scale (ADL) from 
the Groningen Activity Restriction Scale (GARS) ${ }^{52}$ self-efficacy assessed using the 12item Self-efficacy scale ${ }^{5354}$ and autonomy and participation using the questions from the domain Autonomy outdoors from the Impact on Participation and Autonomy questionnaire (IPA). ${ }^{55} 56$

\section{COVARIATES}

Additionally, information on possible confounding factors and effect modifiers was collected. Information on demographic factors (age, gender, marital status, religion, education, occupation) was collected in the screening phase. Other factors measured are: coping using the active coping, avoidant coping and passive coping scales from the Utrecht Coping List (UCL), ${ }^{57}$ mastery using the Personal Mastery Scale developed by Pearlin and Schooler, ${ }^{58}$ anxiety assessed using the anxiety subscale from the Symptom Checklist (SCL-90 $)^{59}$ social support using the short version of the Social Support List- Interactions questionnaire (SSL-I), ${ }^{60}$ co-morbidity using the Chronic conditions list from Statistics Netherlands (CBS - Centraal Bureau voor de Statistiek), life events using a list of 16 life events where patients report which life events they have experienced in the past year, and how they value these events (positive, negative, or neutral). Personality was measured using scales for neuroticism and extraversion from the Eysenck Personality Questionnaire (EPQ), ${ }^{62}$ severity of the chronic illness was assessed using the St. George's Respiratory Questionnaire (SGRQ) for COPD patients, ${ }^{50} 51$ and the Diabetes Symptom Checklist - Revised (DSC-R) for diabetes patients. ${ }^{63}$ If possible, severity of the chronic illness will also be assessed by retrieving lung function (FEV1) and/or blood glucose levels (Hba1c) from hospital records or the general practitioner's records at the end of the study Finally, smoking and body mass index (BMI) were assessed, and in order to check for contamination in the control group, two questions to check whether or not the patients in the control group had heard or benefited from the intervention were added to the questionnaire. Contamination of the control group may lead to a smaller difference in effect between intervention and control group. 
Table 2 Outcome measures and time of assessment in the DELTA study

\begin{tabular}{|c|c|c|c|c|c|c|c|}
\hline \multirow{2}{*}{$\begin{array}{l}\text { Measure } \\
\text { Name questionnaire/variable }\end{array}$} & \multicolumn{7}{|c|}{ Moment in time } \\
\hline & Screening & Baseline & FU1 & FU2 & FU3 & FU4 & Other \\
\hline Marital status/living situation & $\mathrm{x}$ & - & - & - & - & - & - \\
\hline Occupation/work situation & $x$ & - & - & - & - & - & - \\
\hline Education & $x$ & - & - & - & - & - & - \\
\hline Gender & $x$ & - & - & - & - & - & - \\
\hline Age & $x$ & - & - & - & - & - & - \\
\hline Religion & $x$ & - & - & - & - & - & - \\
\hline $\mathrm{BDI}$ & - & $\mathrm{x}$ & $\mathrm{x}$ & $\mathrm{x}$ & - & $\mathrm{x}$ & - \\
\hline Euroqol (QALY's) & - & $x$ & $x$ & $x$ & - & $x$ & - \\
\hline SF36 & - & $x$ & $x$ & $x$ & - & $x$ & - \\
\hline SGRQ & - & $\mathrm{x}$ & $x$ & $\mathrm{x}$ & - & $x$ & - \\
\hline PAID-1 & - & $x$ & $x$ & $x$ & - & $x$ & - \\
\hline ADL-scale from GARS & - & $x$ & $x$ & $x$ & - & $x$ & - \\
\hline $\mathrm{UCL}$ & - & $\mathrm{x}$ & $x$ & $\mathrm{x}$ & - & $x$ & - \\
\hline Personal mastery scale & - & $x$ & $x$ & $x$ & - & $x$ & - \\
\hline Self-efficacy-scale & - & $x$ & $x$ & $x$ & - & $x$ & - \\
\hline IPA & - & $\mathrm{x}$ & $x$ & $\mathrm{x}$ & - & $x$ & - \\
\hline SCL-90 subscale anxiety & - & $x$ & $x$ & $x$ & - & $x$ & - \\
\hline SSL-I 12 & - & $x$ & - & - & - & $x$ & - \\
\hline CBS List Chronic conditions & - & $\mathrm{x}$ & - & - & - & $\mathrm{x}$ & - \\
\hline Diabetes Symptom Checklist-Revised & - & $x$ & $x$ & $\mathrm{x}$ & - & $x$ & - \\
\hline Life-events & - & - & - & - & - & $x$ & - \\
\hline EPQ & - & - & $x$ & - & - & - & - \\
\hline Year of diagnosis DM/COPD & - & $x$ & - & - & - & - & - \\
\hline Smoking & - & - & - & - & - & $x$ & - \\
\hline BMI & - & - & - & - & - & $x$ & - \\
\hline Contamination in control group & - & - & - & - & - & $x$ & - \\
\hline Direct costs within health care system & - & $x$ & $x$ & $x$ & $x$ & $x$ & - \\
\hline Direct costs outside health care system & - & $\mathrm{x}$ & $\mathrm{x}$ & $\mathrm{x}$ & $x$ & $x$ & - \\
\hline Indirect costs outside health care system & - & $x$ & $x$ & $x$ & $x$ & $x$ & - \\
\hline Lung function - if available & - & - & - & - & - & - & $*$ \\
\hline Hba1c - if available & - & - & - & - & - & - & $*$ \\
\hline Process evaluation & - & - & - & - & - & - & + \\
\hline Compliance (in process-evaluation) & - & - & - & - & - & - & $\neq$ \\
\hline
\end{tabular}

FU= follow up

* After intervention

+ During nurses training program and intervention period

‡ During intervention period

\section{Economic evaluation}

A combined cost-effectiveness/ cost-utility analyses will be performed from a societal perspective. The BDI is used as primary outcome measure in the cost-effectiveness analyses. The primary outcomes measure for the cost-utility measure will be utilities based on the social tariff of the EuroQol. ${ }^{64}$ Health care costs, patient and family costs, as well as productivity losses will be recorded using cost diaries. ${ }^{65}$ Patients prospectively kept the diary for two weeks at baseline and for four weeks at each follow up measurement. Afterwards, a telephonist contacted them to retrieve the information from the diary. Data were immediately entered in a computer file to ensure efficiency 


\section{CHAPTER 2}

and reliability. The costs of the intervention were separately calculated. For the valuation of health care costs and patient and family costs, the updated Dutch Guideline for costing in economic evaluations will be used. ${ }^{66}$ If no guideline costs existed, cost prizes were estimated using real costs and tariffs. For future costs and effectiveness data, a discount rate of $4 \%$ will be used.

\section{Process evaluation}

A process evaluation was carried out to assess the following outcomes. The reach of the intervention, defined as the proportion of the intended target population that actually participated in the intervention. The dose delivered was defined as the completeness of the intervention and number and duration of the intervention visit. Dose received, described in two concepts, namely exposure and satisfaction. Exposure is the extent to which patients actively engage with and are receptive to the intervention, and satisfaction is defined as patient's satisfaction with the intervention. ${ }^{67}$ Barriers were described as the extent in which problems were encountered during the intervention.

Data were collected using questionnaires filled out by nurses after every intervention visit, by means of checklists that were kept by the nurse for every patient to report which steps of the intervention had been taken, and by questionnaires filled out by patients after finishing the intervention.

\section{Analysis}

Data will be analysed according to the intention to treat principle. In addition, on treatment analyses will be performed. Changes in primary and secondary outcome measures between intervention and control group will be analysed using both univariate and multivariate techniques. Models will be adjusted for age, gender and socio-economic status (SES), and baseline differences. Potential additional confounding factors and effect modifiers will be checked and, if necessary, included in the model. Since dependency between observations of subjects from the same general practice may exist as well as between repeated observations within persons, multilevel analyses will also be carried out. All analyses will be performed for intervention and control group in total, as well as for DM and COPD separately.

In the economic evaluation the cost and effects of care as usual and MPI by a practice nurse will be calculated and compared. The cost-effectiveness ratio will be stated in terms of costs per improvement on the BDI, the cost-utility ratio will focus on the net cost per QALY gained. Ratios will be determined for the total patient population as well as for COPD or DM patients separately. Bootstrapping will be used to estimate confidence intervals for calculated ratios.

Descriptive statistics, Chi-square and t-tests will be used to analyse data from the process evaluation. 
STUDY DESIGN

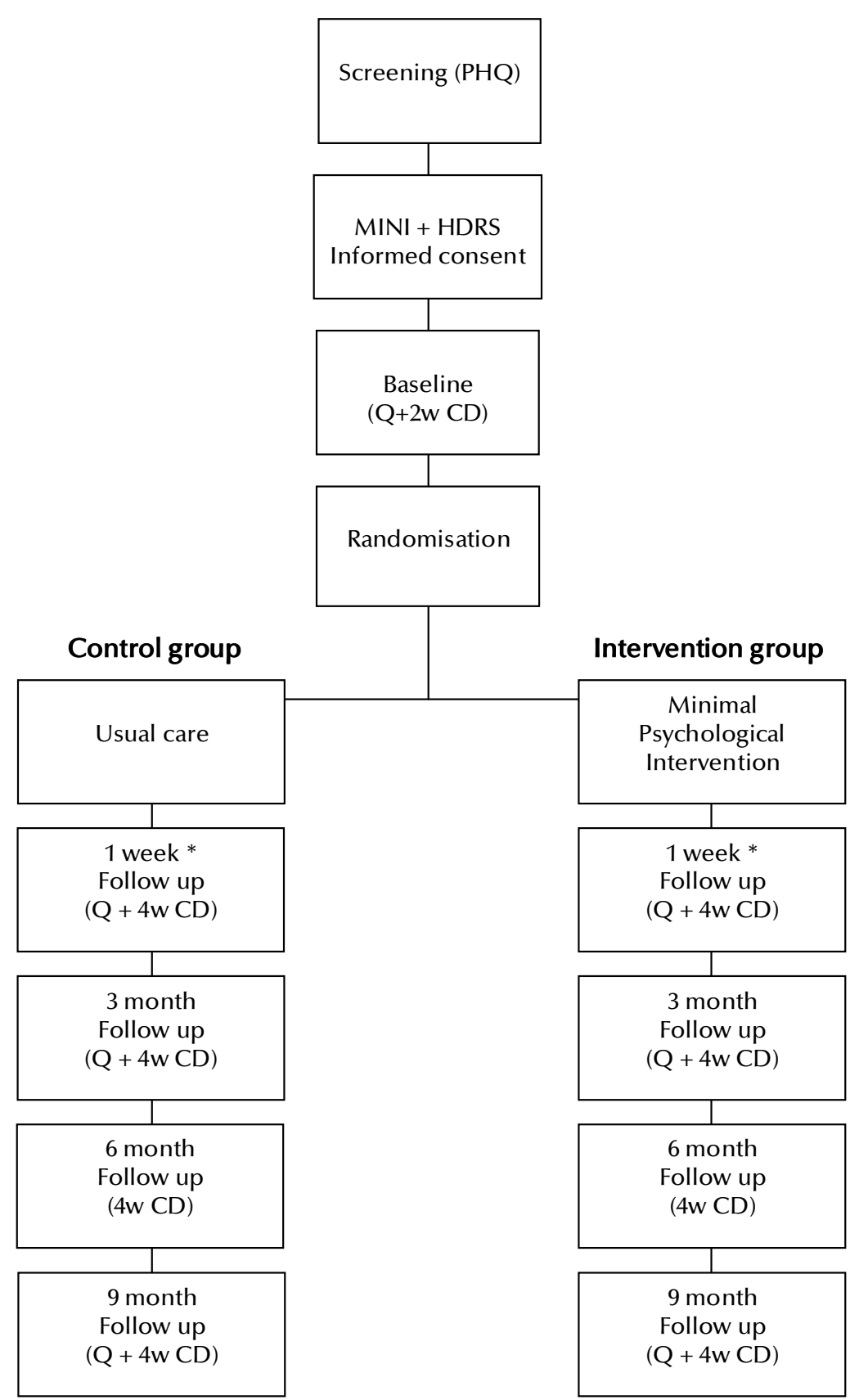

Figure 1 DELTA flowchart

$Q=$ questionnaire, $2 w C D=$ two week cost diary, $4 w C D=$ four week cost diary

* First follow up takes place one week after the intervention period. In the intervention group, this may vary from 2 weeks to three months. In the control group, the intervention period is fixed at six weeks, which is estimated to be the mean duration of the intervention in the intervention group 


\section{CHAPTER 2}

\section{Power calculation}

Assuming an $\alpha$ of 0.05 , a $1-\beta$ (power) of 0.90 , a decrease of 18 percent of non-severe depression in the intervention group versus zero percent in the control group, 192 persons were needed, ${ }^{68} 48 \mathrm{COPD}$ and $48 \mathrm{DM}$ patients in the intervention group and $48 \mathrm{COPD}$ and $48 \mathrm{DM}$ patients in the control group. We decided to recruit four groups of 90 patients (in total: 360), as we not only anticipated the potential need for subgroup specific analyses, but also anticipated attrition varying between 20 and 30 percent (e.g. due to refusals during the follow-up).

\section{DISCUSSION}

\section{Progress of the study}

Based on experiences in the pilot study, we anticipated having to screen 3600 patients in order to include 360 patients. However, we had to increase the number of patients to be screened to reach this number. This was done because the percentage of patients eligible for the MINI interview was lower than in the pilot study. Furthermore, the percentage of patients refusing the MINI interview was higher than expected. To arrive at a gross number of 360 patients we had to screen a total number of 8326 patients. The response rate to the screening questionnaire was $67 \%$. Eventually, 361 non-severely depressed patients were recruited in the study (DM: $N=184$; COPD: $\mathrm{N}=177)$. All interventions have been administered; currently follow-up data are being collected. Data collection will be complete in September 2006.

\section{Process evaluation}

First results of the process evaluation indicate that patients' satisfaction with the intervention is high, and $96.5 \%$ of the patients who received the intervention reported to have benefited from the intervention.

\section{Future implementation}

If this intervention proves to be effective in reducing depression and improving quality of life and proves to be cost-effective, implementation of the intervention in the health care system is considered and anticipated. An implementation and dissemination plan has been developed and is being updated regularly to the latest insights.

\section{Acknowledgements}

We would like to thank Dr. Silvia Evers for her input in the sections about the economic evaluation. This study was funded by the Netherlands Organisation for Health Research and Development (ZonMw), grant number 945-03-047. 


\section{REFERENCES}

1. Pouwer F, Beekman AT, Nijpels G, Dekker JM, Snoek FJ, Kostense PJ, et al. Rates and risks for co-morbid depression in patients with Type 2 diabetes mellitus: results from a community-based study. Diabetologia 2003;46(7):892-8.

2. Bruce DG, Casey GP, Grange V, Clarnette RC, Almeida OP, Foster JK, et al. Cognitive impairment, physical disability and depressive symptoms in older diabetic patients: the Fremantle Cognition in Diabetes Study. Diabetes Res Clin Pract 2003;61(1):59-67.

3. Yohannes AM, Baldwin RC, Connolly MJ. Prevalence of sub-threshold depression in elderly patients with chronic obstructive pulmonary disease. Int J Geriatr Psychiatry 2003;18(5):412-6.

4. van Ede L, Yzermans CJ, Brouwer HJ. Prevalence of depression in patients with chronic obstructive pulmonary disease: a systematic review. Thorax 1999;54(8):688-92.

5. Spitzer RL, Kroenke K, Linzer M, Hahn SR, Williams JB, deGruy FV, 3rd, et al. Health-related quality of life in primary care patients with mental disorders. Results from the PRIME-MD 1000 Study. Jama 1995;274(19):1511-7.

6. Goldney RD, Phillips PJ, Fisher LJ, Wilson DH. Diabetes, depression, and quality of life: a population study. Diabetes Care 2004;27(5):1066-70.

7. Penninx BW, Leveille S, Ferrucci L, van Eijk JT, Guralnik JM. Exploring the effect of depression on physical disability: longitudinal evidence from the established populations for epidemiologic studies of the elderly. Am J Public Health 1999;89(9):1346-52.

8. Katz I-R. On the inseparability of mental and physical health in aged persons: Lessons from depression and medical comorbidity. Am J Geriatr Psychiatry 1996;4(1):1-16.

9. Broadhead WE, Blazer DG, George LK, Tse CK. Depression, disability days, and days lost from work in a prospective epidemiologic survey. Jama 1990;264(19):2524-8.

10. Katon WJ, Lin E, Russo J, Unutzer J. Increased medical costs of a population-based sample of depressed elderly patients. Arch Gen Psychiatry 2003;60(9):897-903.

11. Penninx BW, Guralnik JM, Ferrucci L, Simonsick EM, Deeg DJ, Wallace RB. Depressive symptoms and physical decline in community-dwelling older persons. Jama 1998;279(21):1720-6.

12. Geerlings SW, Beekman AT, Deeg DJ, Van Tilburg W. Physical health and the onset and persistence of depression in older adults: an eight-wave prospective community-based study. Psychol Med 2000;30(2):369-80.

13. Bruce ML, Hoff RA. Social and physical health risk factors for first-onset major depressive disorder in a community sample. Soc Psychiatry Psychiatr Epidemio/1994;29(4):165-71.

14. Bruce ML, Seeman TE, Merrill SS, Blazer DG. The impact of depressive symptomatology on physical disability: MacArthur Studies of Successful Aging. Am J Public Health 1994;84(11):1796-9.

15. Ciechanowski PS, Katon WJ, Russo JE. Depression and diabetes: impact of depressive symptoms on adherence, function, and costs. Arch Intern Med 2000;160(21):3278-85.

16. DiMatteo MR, Lepper HS, Croghan TW. Depression is a risk factor for noncompliance with medical treatment: meta-analysis of the effects of anxiety and depression on patient adherence. Arch Intern Med 2000;160(14):2101-7.

17. Ormel J, Koeter MW, van den Brink W, van de Willige G. Recognition, management, and course of anxiety and depression in general practice. Arch Gen Psychiatry1991;48(8):700-6.

18. Wilson K, Mottram P, Sivanranthan A, Nightingale A. Antidepressant versus placebo for depressed elderly. Cochrane Database Syst Rev 2001(2):CD000561.

19. Ackermann RT, Williams JW, Jr. Rational treatment choices for non-major depressions in primary care: an evidence-based review. J Gen Intern Med 2002;17(4):293-301.

20. Oxman TE, Sengupta A. Treatment of minor depression. Am J Geriatr Psychiatry 2002;10(3):256-64.

21. National Institute for Clinical Excellence. Depression. Managment of depression in primary and secondary care. London: NHS, NICE, 2004.

22. van Marwijk HWJ, Grundmeijer HGLM, Bijl D, van Gelderen MG, de Haan M, van Weel-Baumgarten EM, et al. NHG-standaard Depressieve stoornis (depressie) (eerste herziening). Huisarts Wet 2003;46(11):61423.

23. DeRubeis RJ, Hollon SD, Amsterdam JD, Shelton RC, Young PR, Salomon RM, et al. Cognitive therapy vs medications in the treatment of moderate to severe depression. Arch Gen Psychiatry 2005;62(4):409-16. 


\section{CHAPTER 2}

24. Gloaguen V, Cottraux J, Cucherat M, Blackburn IM. A meta-analysis of the effects of cognitive therapy in depressed patients. J Affect Disord 1998;49(1):59-72.

25. Hollon SD, DeRubeis RJ, Shelton RC, Amsterdam JD, Salomon RM, O'Reardon JP, et al. Prevention of relapse following cognitive therapy vs medications in moderate to severe depression. Arch Gen Psychiatry 2005;62(4):417-22.

26. NIH consensus conference. Diagnosis and treatment of depression in late life. Jama 1992;268(8):1018-24.

27. Lustman PJ, Griffith LS, Freedland KE, Kissel SS, Clouse RE. Cognitive behavior therapy for depression in type 2 diabetes mellitus. A randomized, controlled trial. Ann Intern Med1998;129(8):613-21.

28. Bourbeau J, Nault D, Dang-Tan T. Self-management and behaviour modification in COPD. Patient Educ Couns 2004;52(3):271-7.

29. Moore RG. Improving the treatment of depression in primary care: problems and prospects. $\mathrm{Br} / \mathrm{Gen}$ Pract 1997;47(422):587-90.

30. Mynors-Wallis LM, Gath DH, Day A, Baker F. Randomised controlled trial of problem solving treatment, antidepressant medication, and combined treatment for major depression in primary care. Bmj 2000;320(7226):26-30.

31. Hunkeler EM, Meresman JF, Hargreaves WA, Fireman B, Berman WH, Kirsch AJ, et al. Efficacy of nurse telehealth care and peer support in augmenting treatment of depression in primary care. Arch Fam Med 2000;9(8):700-8.

32. Van Eijk JT, Diederiks JP, Kempen GI, Honig A, Meer Kv K, Brenninkmeijer WJ. Development and feasibility of a nurse administered strategy on depression in community-dwelling patients with a chronic physical disease. Patient Educ Couns 2004;54(1):87-94.

33. Rolland JS. Chronic illness and the life cycle: a conceptual framework. Fam Process 1987;26(2):203-21.

34. Spitzer RL, Kroenke K, Williams JB. Validation and utility of a self-report version of PRIME-MD: the PHQ primary care study. Primary Care Evaluation of Mental Disorders. Patient Health Questionnaire. Jama 1999;282(18):1737-44.

35. Lowe B, Spitzer RL, Grafe K, Kroenke K, Quenter A, Zipfel S, et al. Comparative validity of three screening questionnaires for DSM-IV depressive disorders and physicians' diagnoses. J Affect Disord 2004;78(2):13140 .

36. Kroenke K, Spitzer RL, Williams JB. The PHQ-9: validity of a brief depression severity measure. J Gen Intern Med 2001;16(9):606-13.

37. Sheehan DV, Lecrubier Y, Sheehan KH, Janavs J, Weiller E, Keskiner A, et al. The validity of the Mini International Neuropsychiatric Interview (MINI) according to the SCID-P and its reliability. European Psychiatry 1997;12(5):232-241.

38. Lecrubier Y, Sheehan DV, Weiller E, Amorim P, Bonora I, Sheehan $\mathrm{KH}$, et al. The Mini International Neuropsychiatric Interview (MINI): A short diagnostic structured interview: Reliability and validity according to the CIDI. European Psychiatry1997;12(5):224-231.

39. American Psychiatric Association. DSM-IV-TR: Diagnostic and statistical manual of mental disorders. fourth edition, text revision ed. Washington DC: American Psychiatric Association, 2000.

40. Hamilton M. A rating scale for depression. J Neurol Neurosurg Psychiatry 1960;23:56-62.

41. Hamilton M. Development of a rating scale for primary depressive illness. British journal of social and clinical psychology 1967;6(4):278-96.

42. Lorig K, Gonzalez V. The integration of theory with practice: a 12-year case study. Health Educ $Q$ 1992;19(3):355-68.

43. Goldberg D, Gask L, O'Dowd T. The treatment of somatization: teaching techniques of reattribution. J Psychosom Res 1989;33(6):689-95.

44. van Os TW, Ormel J, van den Brink RH, Jenner JA, Van der Meer K, Tiemens BG, et al. Training primary care physicians improves the management of depression. Gen Hosp Psychiatry 1999;21(3):168-76.

45. Beck AT, Ward CH, Mendelson M, Mock J, Erbaugh J. An inventory for measuring depression. Archives of General Psychiatry 1961;4:561-571.

46. Lustman PJ, Clouse RE, Griffith LS, Carney RM, Freedland KE. Screening for depression in diabetes using the Beck Depression Inventory. Psychosom Med1997;59(1):24-31.

47. Beck AT, Steer RA, Garbin MG. Psychometric properties of the Beck Depression Inventory: Twenty-five years of evaluation. Clinical Psychology Review 1988;8(1):77-100. 
48. Aaronson NK, Muller M, Cohen PD, Essink-Bot ML, Fekkes M, Sanderman R, et al. Translation, validation, and norming of the Dutch language version of the SF-36 Health Survey in community and chronic disease populations. J Clin Epidemio/1998;51(11):1055-68.

49. Snoek FJ, Pouwer F, Welch GW, Polonsky WH. Diabetes-related emotional distress in Dutch and U.S. diabetic patients: cross-cultural validity of the problem areas in diabetes scale. Diabetes Care 2000;23(9):1305-9.

50. Jones PW, Quirk FH, Baveystock CM. The St George's Respiratory Questionnaire. Respir Med 1991;85 Suppl B:25-31; discussion 33-7.

51. Jones PW, Quirk FH, Baveystock CM, Littlejohns P. A self-complete measure of health status for chronic airflow limitation. The St. George's Respiratory Questionnaire. Am Rev Respir Dis 1992;145(6):1321-7.

52. Kempen GI, Sullivan M, van Sonderen E, Ormel J. Performance-based and self-reported physical functioning in low-functioning older persons: congruence of change and the impact of depressive symptoms. J Gerontol B Psychol Sci Soc Sci1999;54(6):P380-6.

53. Bosscher RJ, Smit JH. Confirmatory factor analysis of the General Self-Efficacy Scale. Behav Res Ther 1998;36(3):339-43.

54. Sherer M, et al. The Self-efficacy Scale: Construction and validation. Psychological Reports 1982;51(2):663671.

55. Cardol M, de Haan RJ, van den Bos GA, de Jong BA, de Groot IJ. The development of a handicap assessment questionnaire: the Impact on Participation and Autonomy (IPA). Clin Rehabil1999;13(5):411-9.

56. Cardol M, de Haan RJ, de Jong BA, van den Bos GA, de Groot IJ. Psychometric properties of the Impact on Participation and Autonomy Questionnaire. Arch Phys Med Rehabil 2001;82(2):210-6.

57. Schreurs PJG, Willige van de G, Brosschot JF, Tellegen B, Graus GMH. De Utrechtse Coping Lijs: UCL; Omgaan met problemen en gebeurtenissen. Lisse: Swets en Zeitlinger B.V., 1993.

58. Pearlin LI, Schooler C. The structure of coping. J Health Soc Behav1978;19(1):2-21.

59. Arrindel WA, Ettema JHM. SCL-90: Handleiding bij een multidimensionele psychopathalogie-indicator. Lisse: Swets en Zeitlinger B.V., 1986.

60. Sonderen van E. Het meten van sociale steun met de Sociale Steun Lijst-Interacties (SSL-I) en Sociale Steum Lijst-Discrepanties (SSL-D); een handleiding. Groningen: Noordelijk Centrum voor Gezondheidsvraagstukken (NCG), 1993.

61. Kempen GIJM, Van Eijk LM. The psychometric properties of he SSL12-I, a short scale for measuring social support in the elderly. Social Indicators Research 1995;35(3):303-312.

62. Sanderman R, Arrindell WA, Ranchor AV, Eysenck HJ, Eysenck SBG. Het meten van persoonlijkheidskenmerken met de Eysenck Personality Questionnaire (EPQ): een handleiding. Groningen: Noordelijk centrum voor Gezondheidsvraagstukken, Rijksuniversiteit Groningen, 1995.

63. Grootenhuis PA, Snoek FJ, Heine RJ, Bouter LM. Development of a type 2 diabetes symptom checklist: a measure of symptom severity. Diabet Med1994;11(3):253-61.

64. Dolan P. Modeling valuations for EuroQol health states. Med Care 1997;35(11):1095-108.

65. Goossens ME, Rutten-van Molken MP, Vlaeyen JW, van der Linden SM. The cost diary: a method to measure direct and indirect costs in cost-effectiveness research. J Clin Epidemio/2000;53(7):688-95.

66. Oostenbrink JB, Bouwmans CAM, Koopmanschap MA, Rutten FFH. Handleiding voor kostenonderzoek, methoden en standaard kostprijzen voor economische evaluaties in de gezondheidszorg. Diemen: College voor zorgverzekeringen, Geactualiseerde versie 2004.

67. Linnan L, Steckler A. Process Evaluation for Public Health Interventions and Research: An Overview. In: Steckler A, Linnan L, editors. Process Evaluation for Public Health Interventions and Research. San Francisco: Jossey-Bass, 2002:1-23.

68. Potock S. Clinical trials: A Practical Approach. Chichester: John Wiley and Sons, 1983. 
CHAPTER 2 


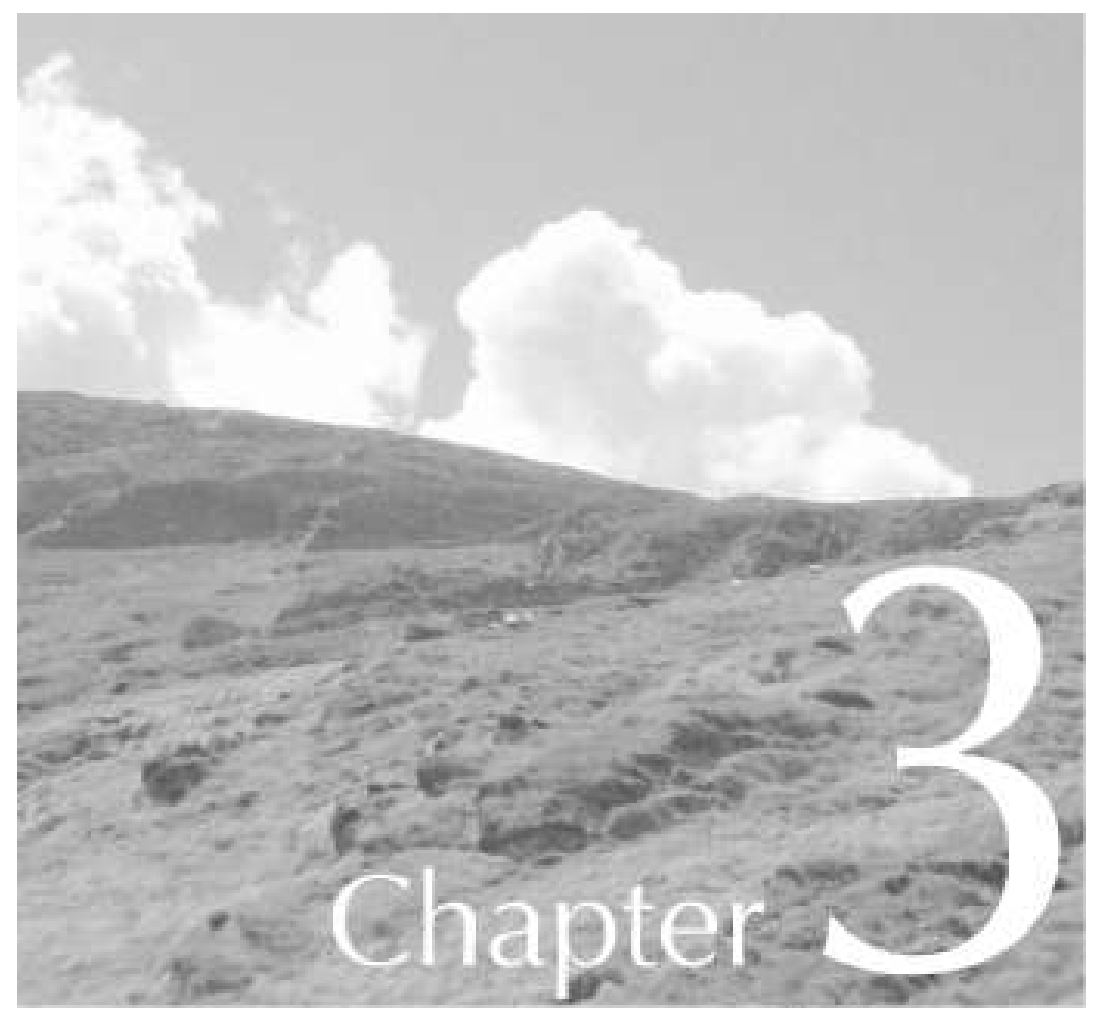

Summed score of the Patient Health Questionnaire-9 was a reliable and valid method for depression screening in chronically ill elderly patients 
Dit hoofdstuk is tot nader bericht van de promovendus verwijderd

- Universiteitsbibliotheek Maastricht - 


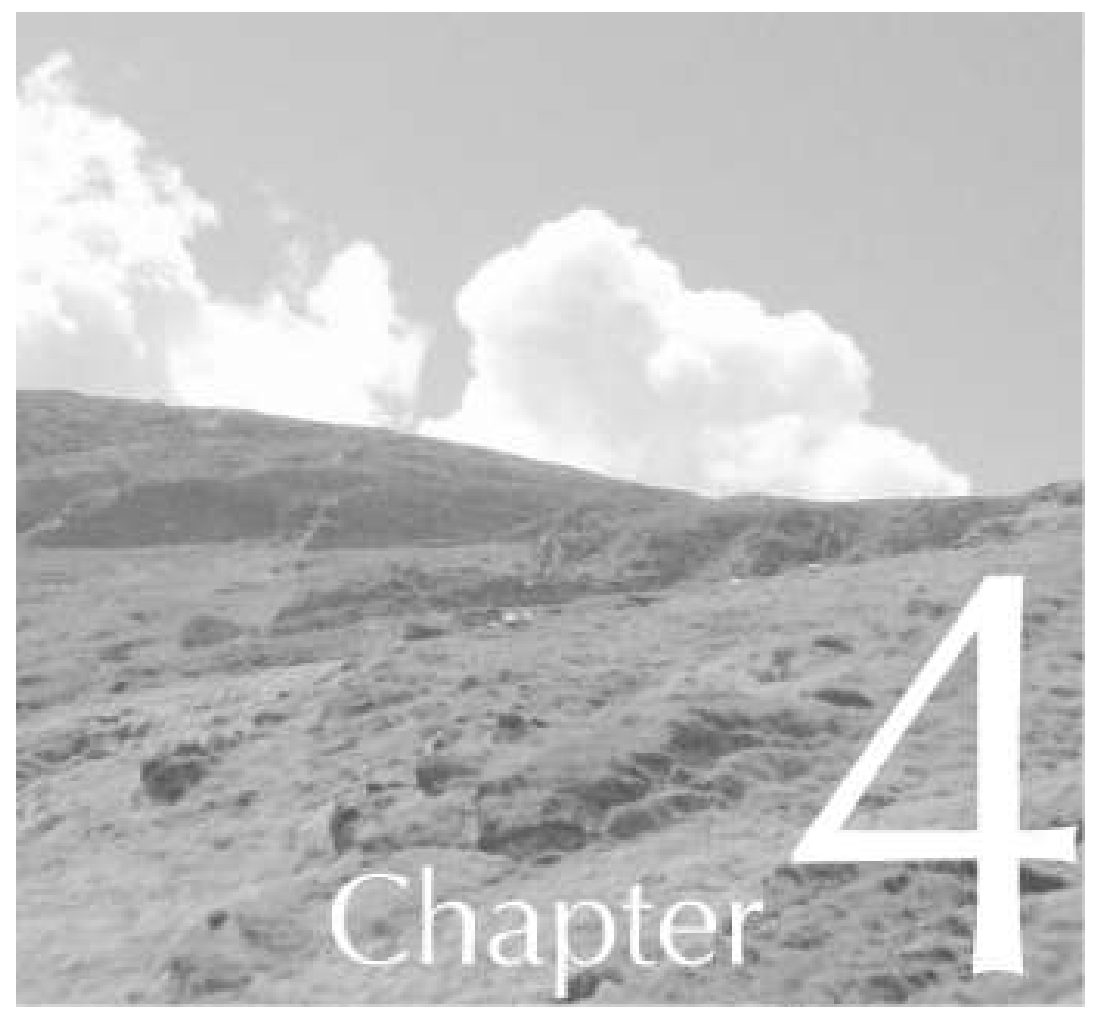

The effectiveness of a minimal psychological intervention in chronically ill elderly patients with depression: a randomised trial (the DELTA-study) 
CHAPTER 4 


\section{ABSTRACT}

Background: Among older persons with chronic somatic diseases, depression often remains unrecognised and untreated in primary care.

Aims: To evaluate the effectiveness of a nurse-led minimal psychological intervention (MPI) in chronically ill elderly persons with depression.

Method: A randomised controlled trial, comparing the MPI with usual care in 361 primary care patients. Patients were 60 years and older and had a minor depression or mild to moderate major depression and either had type II diabetes or chronic obstructive pulmonary disease.

Results: Nine months after the treatment, patients receiving the MPI had fewer depressive symptoms $(\mathrm{p}=0.03)$ and higher odds of a substantial depression reduction $(\geq 50 \%)$ relative to baseline values (OR 3.22; $95 \% \mathrm{Cl} 1.31-7.89)$ than controls. A positive effect was also found on quality of life in diabetic patients.

Conclusions: The nurse-led MPI appears both a feasible and effective treatment for minor to moderate depression in chronically ill elderly persons. 
CHAPTER 4 
Elderly patients with chronic somatic illnesses often have a co-occurring minor or major depression, but such depression often remains unrecognised or untreated in primary care. ${ }^{1}$ As depression may further worsen the patients' prognosis, its treatment in chronically ill elderly patients is important. Several meta-analyses showed that psychological treatments, including cognitive behavioural therapy (CBT), are effective in the treatment of depression in older adults. ${ }^{2-4}$ Two studies on collaborative care further suggest that somatic comorbidity does not adversely affect the positive response $^{5} 6$ and studies on psychological interventions (including CBT) or selfmanagement in chronically ill elderly patients found beneficial effects on various outcomes. ${ }^{7-12}$ Recent studies show that paraprofessionals, like nurses, can successfully be trained to administer forms of CBT and self-management strategies and thus may complement the general practitioner's (GP) regular work. ${ }^{13} 14$ The aim of the DELTA study (Depression in Elderly with Long-Term Afflictions) was to evaluate the effectiveness of a nurse-administered Minimal Psychological Intervention (MPI) in reducing depressive symptoms in elderly primary care patients with type II diabetes mellitus (DM) or chronic obstructive pulmonary disease (COPD) and a co-occurring non-severe depression.

\section{METHODS}

\section{Design}

The DELTA study (Depression In Elderly with Long-Term Afflictions) was a two-armed, pragmatic, randomised controlled trial with a baseline measurement (before randomisation) and three follow-up measurements at one week and at three and nine months after the intervention period (trial registration: ISRCTN92331982). In contrast to an explanatory trial in which a treatment is compared to a placebo under ideal circumstances, we conducted a pragmatic trial, measuring effectiveness of the MPI in a setting that realistically reflects routine clinical practice. To achieve this realistic setting, we included a heterogenic population, consisting of both DM and COPD patients with depression severities that - if detected and treated - most commonly would be treated in primary care. The intervention group received the MPI, while the control group received care as usual. Time between baseline and the first-follow-up measurement depended upon the time it took to complete the tailor-made intervention. In the control group, the first follow-up measurement was set at six weeks after baseline, which beforehand was estimated the mean duration of the intervention (in the intervention group).

A block randomisation scheme was used with stratification for chronic illness and general practice. The block size was two, because we expected to include a small number of patients per general practice and wanted to have them equally distributed over groups. The researchers entered patients in a computer connected to an external agency, which performed the randomisation using a computerised random 
number generator. All data were collected through self-administered questionnaires. Data entry was performed by researchers blinded for the allocation. Approval for the study protocol was obtained from the Medical Ethics Committee of Maastricht University / University Hospital Maastricht. A detailed description of the study protocol has been published elsewhere. ${ }^{15}$

We hypothesized that the MPI, based on the principles of CBT and self-management, would be more effective in reducing symptoms of depression and increasing quality of life than care as usual. We also examined whether the type of chronic illness (DM or COPD) modified the effects of the MPI. Since patients with different chronic illnesses probably face similar problems in coping with their disease (or its consequences) in daily life, ${ }^{1617}$ we expected that the effect of the intervention would be generic across diseases.

\section{Participants and setting}

Between October 2003 and May 2005, participants with DM or COPD were recruited in 89 primary care practices in the south of the Netherlands. The decision to include DM and COPD patients was based on the fact that these prevalent diseases are mainly treated in primary care in the Netherlands. Patients who had been diagnosed by their GP with type II diabetes mellitus or COPD, who were aged 60 years or over, who were community-dwelling and who did not meet exclusion criteria (treatment with antidepressants for depression, major psychiatric problems (bipolar depression, schizophrenia, alcohol or substance abuse), current psychosocial/psychiatric treatment, serious cognitive problems, on waiting list for nursing home, bedridden, loss of spouse within last three months, and not being fluent in Dutch) were sent the Patient Health Questionnaire (PHQ-9). ${ }^{18}$ Patients who reported having at least two symptoms present for more than half of the days, one of them being loss of interest or depressed mood, were invited to a structured diagnostic interview for DSM-IV axis I disorders, the Mini International Neuropsychiatric Interview (MINI). ${ }^{19}$ The MINI was administered at the patients' home by nurses who had been trained by a psychiatrist and a GP, and had regular booster sessions with the psychiatrist. Patients with minor depression, mild to moderate major depression or dysthymia, according to the MINI, were invited to participate in the trial. Patients with suicidal risk and patients with severe major depression, according to the Hamilton Depression Rating Scale (HDRS $>18),{ }^{20}$ were excluded and referred to their GP. Somatic symptoms on the Hamilton scale were assessed using an 'etiologic' approach; symptoms that could be accounted for by the chronic somatic illness would not be counted towards the depression. This is a recommended approach for the assessment of depression in persons with a physical illness. ${ }^{21}$ After signing an informed consent form and completing a baseline questionnaire, patients were randomly allocated to the MPI or to care as usual. In all, 361 patients (185 DM; 176 COPD) were randomised. Multimorbidities are common in elderly persons, as was indeed the case in our study: 11 
patients stratified for DM also had COPD, and 23 patients stratified for COPD also had DM.

\section{Interventions}

Patients allocated to the intervention group received the MPI additionally to usual care. Table 1 lists the phases of the intervention; more details can be found elsewhere. ${ }^{1522}$ The intervention aims to educate patients to take responsibility for day-today management of their own illness and its consequences. The intervention was delivered at the patient's home by trained nurses. The nurse delivering the intervention was a different nurse than the one administering the MINI. The intervention is a combination of CBT and self-management; reattribution of negative cognitions and problem solving were core elements of the intervention. The intervention is tailormade; during the study, patients received two to ten visits over a period of at most three months. The number of visits depended on their progress through the steps of the intervention; on average patients received four intervention visits, each lasting approximately one hour. On average, patients in the intervention group completed the intervention in ten weeks (mean 10.3 (SD 5.6)). To ensure that the nurses adhered to the protocol during the study, they were asked to keep checklists, covering all essential intervention steps, for each patient. Examination of these lists showed that nurses had adhered closely to the guidelines in the protocol. ${ }^{23}$ Patients allocated to the control group received care as usual for their somatic illness, according to the clinical guidelines of the Dutch College of General Practitioners. These guidelines encompass regular check-up of medical symptoms, but do not explicitly involve detection and treatment of depressive symptoms. ${ }^{24-26}$ Depression treatment next to the MPI during follow-up was uncommon and non-differential between the intervention and control group. At the last follow-up phase, seven and one person in the control group received antidepressants or consulted a psychiatrist or psychologist, respectively, compared with four and six persons in the intervention group. Only after the follow-up, GPs were informed about which patients had participated in the trial.

Table 1 Phases of the Minimal Psychological Intervention

\begin{tabular}{ll}
\hline Phase & Description \\
\hline 1 & The nurse explores the patient's feelings, cognitions and behaviours \\
2 & $\begin{array}{l}\text { The patient keeps a diary, where he or she records symptoms, complaints, thoughts, worries, related } \\
\text { feelings, and behaviour }\end{array}$ \\
3 & $\begin{array}{l}\text { The patient is challenged to link his or her mood to the consequent behaviour, using information from } \\
\text { the diary }\end{array}$ \\
& $\begin{array}{l}\text { The self-management approach is introduced. The patient explores possibilities to alter his or her } \\
\text { behaviour and draws up an action plan }\end{array}$ \\
& Evaluation of progress in achieving the goals of the action plan
\end{tabular}




\section{CHAPTER 4}

\section{Main outcome measures}

The primary outcome measure was depression, using the Beck Depression Inventory (BDI). ${ }^{27} 28$ In addition to the continuous BDI score, a dichotomous BDI score to represent clinically relevant improvement was calculated. Improvement of depression was defined as an $\geq 50 \%$ reduction relative to the baseline BDI score. ${ }^{29}$ The secondary outcome measure was quality of life, using the Physical Component Score (PCS) and Mental Component Score (MCS) of the Short-Form 36 (SF-36). ${ }^{30}$ Missing items on the SF-36 and on the BDI were imputed using the individual mean score of items that were not missing in patients for whom at least $50 \%$ of items were available. The number of patients in whom imputation was not possible across measurements (T0T1-T2-T4) was two-zero-two-two for the BDI and 47-22-16-21 for the PCS and MCS; this was equally distributed over groups. Data on age, sex, and education, as covariates, were collected during the PHQ-9 screening.

\section{STATISTICAL ANALYSIS}

Assuming an $\alpha$ of 0.05 , a $1-\beta$ (power) of 0.90 , including $2 \times 96=192$ patients (48 COPD and $48 \mathrm{DM}$ patients in the intervention group and 48 COPD and 48 DM patients in the control group) would allow a minimum difference between groups of $18 \%$ in improvement $\left(\geq 50 \%\right.$ reduction relative to the baseline BDI score) to be detected. ${ }^{31}$ These number would further allow the detection of a difference in BDI score of at least 3.57 points at the $5 \%$ significance level to be detected (power 0.9). Anticipating an attrition rate of approximately $30 \%$ and the potential need for sub-group analyses, we decided to recruit a gross number of 360 patients.

All analyses were carried out according to a pre-established analysis plan on an intention-to-treat basis. Chi-square and t-tests were used to test the comparability of groups at baseline in terms of all outcomes and demographic variables. We used a mixed-model, repeated-measures ANCOVA to test for differences between groups at different points in time. We included seven fixed effects in our model (age, sex, education level, baseline value, treatment group, time, and the interaction term between treatment group and time). There was no support for general practice being an additional level. Several random effects and covariance matrices were then tested, using -2 log likelihood tests to decide which model had the best fit. Lastly, two-way and three-way interaction terms for group, time, and illness (DM or COPD) were used to test for effect modification by disease $(p<0.10)$. A check for outliers was conducted, but no serious outlier influence was established. Percentages of improvement of depression score were calculated for both groups, and between-group differences were tested using Chi-square tests. Logistic regression was used to calculate odds ratios (ORs) for improvement, correcting for age, sex, level of education, time, and BDI baseline score. Additional per-protocol analyses were performed with intervention patients who - according to an analysis of process data ${ }^{23}$ - had received the 
complete intervention $(n=136)$ and excluding nine control patients who responded positively to a check for contamination during the data collection. Finally, sensitivity analyses using the last value carried forward-method were done. These analyses gave similar results.

\section{RESULTS}

Figure 1 describes the patient flow and follow-up. In total, 8,326 patients with DM or COPD received the screening questionnaire, $67 \%$ of whom returned the questionnaire. Nine hundred and sixty-five patients were eligible for the diagnostic interview (17.3\%). Of these patients, 221 (23\%) refused to participate in the MINI or could not be reached. A comparison of participants $(n=744)$ and non-participants $(n=221)$ revealed no differences between these groups regarding gender, age, educational level, or PHQ-9 sum score. The 83 patients that were eligible, but who refused to participate in the trial, were more often female, older and were significantly lower educated. In total, 361 patients were randomised; 183 to the intervention and 178 to usual care.

During the follow-up period, we had a dropout rate of $33 \%$, as anticipated. The dropout rate was somewhat higher, but not significantly, in the intervention group than in the control group $(37.7 \%$ vs. $29.8 \%$, p-value 0.11$)$. Overall, dropouts were on average 2.8 years older than non-dropouts ( $p$-value $<0.00$ ), but they did not differ in baseline BDI depression scores (p-value 0.11). As shown in Table 2, the intervention and control groups were comparable in terms of baseline characteristics. 


\section{CHAPTER 4}

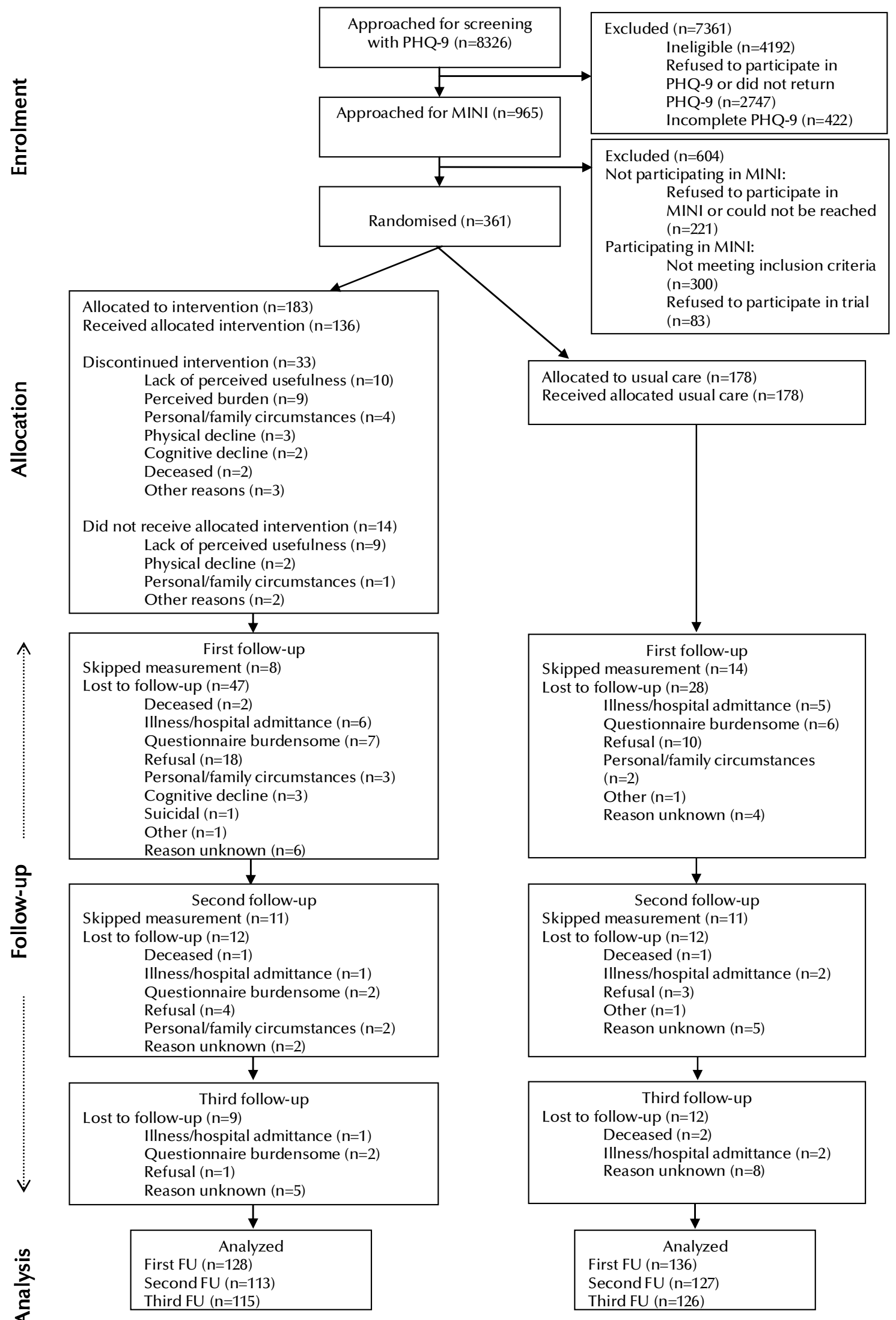

Figure 1 Patient flow and follow-up in the DELTA study

Legend: First follow-up at one week after the intervention, second follow-up at three months after the intervention and third follow-up at nine months after the intervention. 
EFFECT EVALUATION

Table 2 Comparability of intervention and control groups in terms of socio-demographic variables and baseline values of outcomes

\begin{tabular}{|c|c|c|c|c|}
\hline Variable & & $\begin{array}{l}\text { Usual Care } \\
\text { n=178 }\end{array}$ & $\begin{array}{c}\text { MPI } \\
n=183\end{array}$ & P-value \\
\hline Age, yrs (SD) & & $70.6(6.8)$ & $70.8(6.5)$ & .73 \\
\hline \multicolumn{5}{|l|}{ Sex, No. (\%) } \\
\hline & Male & $95(53.4)$ & $98(53.6)$ & \multirow[t]{2}{*}{.97} \\
\hline & Female & 83 (46.6) & $85(46.4)$ & \\
\hline \multicolumn{5}{|c|}{ Chronic illness, No. (\%) } \\
\hline & Diabetes & $94(52.8)$ & $91(49.7)$ & \multirow[t]{2}{*}{.56} \\
\hline & COPD & $84(47.2)$ & $92(50.3)$ & \\
\hline \multicolumn{5}{|c|}{ Education level ${ }^{*^{\dagger}}$, No. (\%) } \\
\hline & Low & $60(33.9)$ & $64(35.4)$ & \multirow[t]{3}{*}{.55} \\
\hline & Medium & $46(26.0)$ & $54(29.8)$ & \\
\hline & High & $71(40.1)$ & $63(34.8)$ & \\
\hline BDI, mean (SD) & & $17.7(8.0)$ & $17.1(7.2)$ & .47 \\
\hline PCS, mean (SD) & & $34.2(9.1)$ & $34.4(9.5)$ & .81 \\
\hline MCS, mean (SD) & & $36.9(9.9)$ & $37.8(8.2)$ & .38 \\
\hline \multicolumn{5}{|c|}{$\begin{array}{l}\text { Low refers to primary school only, medium refers to lower vocational training or lower general } \\
\text { education, high refers to higher vocational training, secondary school, higher professional education } \\
\text { and university training }\end{array}$} \\
\hline \multicolumn{5}{|c|}{$\begin{array}{l}\text { Range of the BDI is } 0-63, \text { with } 0 \text { as the most favourable outcome; PCS and MCS are standardised scores } \\
\text { with a mean of } 50 \text { and a standard deviation of } 10 \text { in the US general population; higher scores are more } \\
\text { favourable }\end{array}$} \\
\hline
\end{tabular}

Results from the mixed-model ANCOVA analyses (Table 3) show that the intervention group had lower average BDI scores than the control group, indicating fewer depressive symptoms, and this was consistently found at all three follow-up measurements. The difference between the intervention and control groups was significant at the second follow-up (mean BDI difference 1.61, $\mathrm{F}=3.860, \mathrm{df}=1,510, \mathrm{p}$-value $<0.05$ ) and the last follow-up (mean BDI difference 2.09, $F=4.998, \mathrm{df}=1,270$, $\mathrm{p}$-value 0.03). The intervention group consistently had higher scores for PCS and MCS than the control group, indicating better quality of life, but these differences did not reach statistical significance. A marginal difference for PCS was found at the second follow-up $(\mathrm{F}=3.326, \mathrm{df}=1,450$, $\mathrm{p}$-value 0.07$)$. 
Table 3 Outcomes on depression and quality of life at follow-up

\begin{tabular}{|c|c|c|c|c|c|c|}
\hline & $\begin{array}{l}\text { Usual Care } \\
\qquad \mathrm{N}=178\end{array}$ & & $\begin{array}{c}\mathrm{MPI} \\
\mathrm{N}=183\end{array}$ & & & $\begin{array}{l}\text { Mean difference } \\
\text { (Usual care - MPI) }\end{array}$ \\
\hline & Mean $(\mathrm{SE})^{*}$ & $\mathrm{~N}^{+}$ & Mean $(\mathrm{SE}) *$ & $\mathrm{~N}^{+}$ & P-value & Mean $(95 \% \mathrm{Cl})^{*}$ \\
\hline \multicolumn{7}{|l|}{$\overline{\mathrm{BDI}}$} \\
\hline After 1 week & $17.17(0.53)$ & 135 & $16.18(0.55)$ & 127 & .19 & $0.99(-0.50$ to 2.49$)$ \\
\hline After 3 months & $17.49(0.56)$ & 125 & $15.88(0.60)$ & 111 & $<.05$ & $1.61(>0.00$ to 3.22$)$ \\
\hline After 9 months & $18.00(0.64)$ & 123 & $15.91(0.68)$ & 114 & .03 & $2.09(0.25$ to 3.93$)$ \\
\hline \multicolumn{7}{|l|}{$\overline{\mathrm{PCS}}$} \\
\hline After 1 week & $33.60(0.57)$ & 109 & $34.26(0.58)$ & 110 & .42 & $-0.66(-2.25$ to 0.94$)$ \\
\hline After 3 months & $33.31(0.61)$ & 102 & $34.89(0.62)$ & 101 & .07 & -1.58 (-3.28 to 0.12$)$ \\
\hline After 9 months & $33.35(0.70)$ & 98 & $34.15(0.70)$ & 103 & .43 & $-0.79(-2.74$ to 1.16$)$ \\
\hline \multicolumn{7}{|l|}{ MCS } \\
\hline After 1 week & $38.30(0.86)$ & 109 & $39.84(0.86)$ & 110 & .21 & $-1.54(-3.92$ to 0.84$)$ \\
\hline After 3 months & $38.15(0.88)$ & 102 & $39.06(0.89)$ & 101 & .47 & $-0.90(-3.36$ to 1.55$)$ \\
\hline After 9 months & $37.56(0.89)$ & 98 & $39.43(0.88)$ & 103 & .14 & $-1.87(-4.34$ to 0.59$)$ \\
\hline
\end{tabular}

Abbreviations: SE, standard error; 95\%Cl, 95\% confidence interval; BDI, Beck Depression Inventory; PCS, Physical Component Score; MCS, Mental Component Score.

Range of the BDI is 0-63, with 0 as the most favourable outcome; PCS and MCS are standardised scores with a mean of 50 and a standard deviation of 10 in the US general population; higher scores are more favourable.

*estimates from mixed-model regression analyses, corrected for age, sex, level of education, and baseline value of the outcome measure.

${ }^{+}$Data on education was missing for 2 persons

No significant two-way or three-way product terms of disease with group and time were found in the analyses with BDI and PCS (data not shown). For MCS, there was a significant product term with disease. The group $x$ disease term was significant ( $p$ value 0.08), indicating a different effect of the intervention in DM patients than in COPD patients. Table 4 presents the results of the MCS analyses stratified by disease. The stratified analyses revealed that in DM patients, the intervention group had higher overall scores on the MCS than the control group, with a statistically significant difference at the last follow-up (mean MCS difference 3.85, $\mathrm{F}=5.324, \mathrm{df}=1,268.62$, p-value 0.02). For COPD patients, no effect on MCS was found.

Table 5 shows improvement rates in depressive symptoms ( $\geq 50$ reduction relative to baseline BDI score) and ORs for both groups. The intervention group had a significantly higher improvement rate at the last follow-up than the control group, with a significant odds ratio (OR 3.22; 95\% Cl 1.31-7.89). No interaction between group and disease was found (data not shown). 
EFFECT EVALUATION

Table 4 Outcomes of disease-stratified analyses of the Mental Component Scores

\begin{tabular}{|c|c|c|c|c|c|c|c|}
\hline & & Usual Care & & MPI & & & Mean difference \\
\hline & & Mean $(\mathrm{SE}) *$ & $\mathrm{~N}$ & Mean $(\mathrm{SE}) *$ & $\mathrm{~N}$ & $\mathrm{P}$-value & (Usual care-MPI) \\
\hline \multirow[t]{4}{*}{$\overline{\mathrm{DM}}$} & & & & & & & Mean $(95 \% \mathrm{Cl})$ \\
\hline & After 1 week & $37.47(1.18)$ & 54 & $40.07(1.19)$ & 54 & .12 & $-2.60(-5.90$ to 0.70$)$ \\
\hline & After 3 months & $37.15(1.17)$ & 55 & $39.94(1.21)$ & 52 & .10 & $-2.80(-6.11$ to 0.51$)$ \\
\hline & After 9 months & $35.87(1.17)$ & 55 & $39.72(1.19)$ & 54 & .02 & $-3.85(-7.14$ to -0.57$)$ \\
\hline \multicolumn{8}{|c|}{ COPD } \\
\hline & After 1 week & $39.20(1.25)$ & 55 & $39.70(1.22)$ & 56 & .78 & $-0.50(-3.95$ to 2.95$)$ \\
\hline & After 3 months & $39.35(1.33)$ & 47 & $38.22(1.30)$ & 49 & .55 & $1.12(-2.55$ to 4.80$)$ \\
\hline & After 9 months & $39.67(1.37)$ & 43 & $39.22(1.29)$ & 49 & .81 & $0.45(-3.27$ to 4.17$)$ \\
\hline
\end{tabular}

Abbreviations: SE, standard error; 95\%Cl, 95\% confidence interval; DM, type // diabetes mellitus; COPD, chronic obstructive pulmonary disease

MCS is a standardised score with a mean of 50 and a standard deviation of 10 in the US general population; higher scores are more favourable.

*estimates from mixed-model regression analyses, corrected for age, sex, level of education, and baseline value of MCS.

Table 5 Percentages and odds ratios for improvement* on the depression outcome.

\begin{tabular}{lccc}
\hline \multicolumn{3}{c}{ BDI improvement rate } \\
\hline & Usual care & $\mathrm{MPI}$ & OR (95\%Cl)† \\
\hline After 1 week & $\mathrm{N}(\%)$ & $8(6.3)$ & $0.58(0.18-1.88)$ \\
After 3 months & $10(7.4)$ & $14(12.4)$ & $1.49(0.58-3.84)$ \\
After 9 months & $11(8.7)$ & $20(17.5)^{\ddagger}$ & $3.22(1.31-7.89)$ \\
\hline
\end{tabular}

*defined as a reduction of baseline BDI score by at least $50 \%$

tfrom logistic regression corrected for age, sex, educational level, time, and baseline BDI value; control group is reference category

${ }^{\ddagger}$ P value.02

\section{DISCUSSION}

Our nurse-led intervention significantly reduced depressive symptoms in chronically ill elderly patients with minor or mild to moderate major depression at three and nine months after the intervention. BDI improvement rates were significantly higher in the intervention patients than in the controls at the last follow-up. Although we expected effects on quality of life in all patients, a significant effect was only found on the mental component score in DM patients at the last follow-up.

Several aspects should be taken into account when interpreting our results. First, as expected, we had an overall dropout rate of $33 \%$, with slightly more dropouts in the intervention group than in the control group, but this difference was not significant. In the analyses, however, we used a mixed model for repeated measures, which uses all available data for each patient. Although we can not completely rule out bias from attrition, the absence of differences in baseline BDI depression scores between dropouts and non-drop-outs and the fact that intervention and control group continued to be comparable over time, does not make attrition bias plausible. Second, blinding of 
patients obviously was not possible, as we compared the intervention with usual care, as is often done in pragmatic trials. ${ }^{32}$

The results of the depression outcome are in line with outcomes of previous studies evaluating psychological interventions in chronically ill elderly patients. Several smaller trials found that $\mathrm{CBT}^{9}{ }^{9}$ interpersonal counselling, ${ }^{8}$ and self-management, ${ }^{12}$ reduced depression in chronically ill elderly, but one study only evaluated short-term effects and included both depressed and non-depressed patients. ${ }^{9}$ The PEARLS study evaluated a program including Problem Solving Therapy in chronically ill elderly persons with minor depression or dysthymia and found an OR of 5.2 for a $50 \%$ improvement of the depression score. ${ }^{7}$ Neither of the abovementioned studies, however, were performed in primary care settings, nor did these studies assign primary care nurses for the administration of the intervention.

In our study, differences between groups in BDI score were relatively small, as were improvement rates. This illustrates how persistent depressive symptoms in elderly populations are. ${ }^{33}$ Nevertheless, BDI effect sizes were 0.26 at the second and 0.29 at the third follow-up (not tabulated), which can be regarded as small to medium-sized effects. ${ }^{34}$ Furthermore, the OR for BDI improvement at the last follow-up revealed a threefold higher probability of improvement in intervention patients than in controls. Also, in patient with diabetes, there were consistent positive intervention effects on quality of life, which reached statistical significance at the last follow-up, with a medium effect size of 0.45 (not tabulated).

According to some, much of the effect of psychological treatments can be attributed to the effects of patients receiving extra attention. ${ }^{4}$ However, if the effects of our intervention were solely attributable to the extra attention, we would have expected to see an effect immediately at the first follow-up. However, we only found effects in the longer term. Since our intervention is a short cognitive behavioural skill-based program, the most plausible explanation is that patients gained skills and continued to apply them in daily life during the follow-up period. As a result, their depression did not deteriorate during follow-up, and as depressive symptoms in the control group slightly deteriorated over time, this led to significant differences at later followup. It is not unlikely, that intervention patients will continue to improve over a longer time interval if patients continue to increase their skills and become more confident self managers. Two comparable studies also found that effects became more pronounced during longer follow-up. ${ }^{811} 12$

Trained nurses played a major role in our study, both in diagnosing depression and in administering the intervention at the patient's home. However, incorporating the intervention in the disease and care management programs of primary care facilities in the Dutch setting will probably mean that patients will not be visited at home. Since patients with chronic illnesses already come to primary care facilities for their regular check-ups, this should not represent a barrier to patients. Furthermore, having practice nurses deliver the intervention has several advantages: they see individual patients on a regular basis, they are aware of the patients' personal circumstances and health-related problems, they can be trained to assess depression using 
diagnostic interviews, ${ }^{35}$ and more importantly, they can monitor a possible relapse of depression and take action if necessary.

With the aging of the population in Western society, the number of elderly patients with a chronic illness is expected to increase. This will put further pressure on health care systems in general, and on primary care, in particular. It is important that patients do not fall victim to the negative consequences of a comorbid depression as well, as this may have a substantial adverse impact on the course of their somatic illness and hence on their quality of life. Therefore, early detection of depressive disorders in chronically ill elderly persons is important and screening methods should be incorporated in their regular care. Minimal interventions like our MPI, which (1) provide patients with the skills to cope with the consequences of their illness and their depressive symptoms, (2) can be incorporated in existing disease and care management programs, and (3) can be administered by nurses (e.g. practice nurses), can play a major role in preventing further deterioration of patients and may help alleviate the burden on the health care system. The modest, but positive results from the current effectiveness study, and the positive results from an extensive process evaluation, revealing high patient satisfaction, ${ }^{23}$ strengthen our belief that the DELTA intervention may be well suited for implementation within existing disease and care management programs for DM and COPD in primary care.

\section{CONCLUSION}

Our relatively short psychological intervention, administered by a nurse, reduced depressive symptoms in chronically ill elderly patients with minor depression and mild to moderate major depression and improved quality of life in elderly DM patients. The intervention thus appears to have a generic effect on depression, but the effect on quality of life might be disease-specific. Given the findings from the current effectiveness-study (medium effect sizes and a pattern of increasing effects over longer time) and the positive results from process evaluation, opportunities for implementing the intervention in existing disease management programs should be explored, while awaiting results from the economic evaluation. Furthermore, patient characteristics and traits that predict response to treatment should be identified and recognition of depression in chronically ill elderly people in primary care should be further improved. To conclude, our intervention appears an effective and acceptable treatment for minor depression and mild to moderate major depression and may be a valuable addition to existing disease management programs for older patients. 


\section{CHAPTER 4}

\section{Acknowledgements}

We thank Kitty Daemen, Henny Geelen, Francine Hendriks, and Mieke Witte, for administering the intervention with dedication and enthusiasm, Wendy Engering for her contribution to the patient recruitment and Dorien Mintjes for her contribution to the data entry, as well as MEMIC (Centre for Data and Information Management, Maastricht University) for providing us with a data management system. This study was funded by the Netherlands Organisation for Health Research and Development (ZonMw), Health Care Efficiency Research Program, grant number 945-03-047. 
EFFECT EVALUATION

\section{REFERENCES}

1. Djernes JK. Prevalence and predictors of depression in populations of elderly: a review. Acta Psychiatr Scand 2006;113(5):372-87.

2. Cuijpers P, van Straten A, Smit F. Psychological treatment of late-life depression: a meta-analysis of randomized controlled trials. Int. J. Geriatr. Psychiatry 2006;21(12):1139-49.

3. Pinquart $M$, Duberstein PR, Lyness JM. Treatments for later-life depressive conditions: a meta-analytic comparison of pharmacotherapy and psychotherapy. Am. J. Psychiatry 2006;163(9):1493-501.

4. McCusker J, Cole M, Keller E, Bellavance F, Berard A. Effectiveness of treatments of depression in older ambulatory patients. Arch. Intern. Med. 1998;158(7):705-12.

5. Bogner HR, Cary MS, Bruce ML, Reynolds CF, 3rd, Mulsant B, Ten Have T, et al. The role of medical comorbidity in outcome of major depression in primary care: the PROSPECT study. Am J Geriatr Psychiatry 2005;13(10):861-8.

6. Harpole LH, Williams JW, Jr., Olsen MK, Stechuchak KM, Oddone E, Callahan CM, et al. Improving depression outcomes in older adults with comorbid medical illness. Gen Hosp Psychiatry 2005;27(1):4-12.

7. Ciechanowski P, Wagner E, Schmaling K, Schwartz S, Williams B, Diehr P, et al. Community-integrated home-based depression treatment in older adults: a randomized controlled trial. JAMA 2004;291(13):156977.

8. Mossey JM, Knott KA, Higgins M, Talerico K. Effectiveness of a psychosocial intervention, interpersonal counseling, for subdysthymic depression in medically ill elderly. J. Gerontol. A. Biol. Sci. Med. Sci. 1996;51(4):M172-8.

9. Kunik ME, Braun U, Stanley MA, Wristers K, Molinari V, Stoebner D, et al. One session cognitive behavioural therapy for elderly patients with chronic obstructive pulmonary disease. Psychol. Med. 2001;31(4):717-23.

10. Emery CF, Schein RL, Hauck ER, Maclntyre NR. Psychological and cognitive outcomes of a randomized trial of exercise among patients with chronic obstructive pulmonary disease. Health Psychol. 1998;17(3):232-40.

11. Brody BL, Roch-Levecq AC, Thomas RG, Kaplan RM, Brown SI. Self-management of age-related macular degeneration at the 6-month follow-up: a randomized controlled trial. Arch Ophthalmo/2005;123(1):46-53.

12. Brody BL, Roch-Levecq AC, Kaplan RM, Moutier CY, Brown SI. Age-related macular degeneration: selfmanagement and reduction of depressive symptoms in a randomized, controlled study. J Am Geriatr SoC 2006;54(10):1557-62.

13. Hunkeler EM, Meresman JF, Hargreaves WA, Fireman B, Berman WH, Kirsch AJ, et al. Efficacy of nurse telehealth care and peer support in augmenting treatment of depression in primary care. Arch Fam Med 2000;9(8):700-8.

14. Mynors-Wallis LM, Gath DH, Day A, Baker F. Randomised controlled trial of problem solving treatment, antidepressant medication, and combined treatment for major depression in primary care. BMJ 2000;320(7226):26-30.

15. Lamers F, Jonkers CC, Bosma H, Diederiks JP, van Eijk JT. Effectiveness and cost-effectiveness of a minimal psychological intervention to reduce non-severe depression in chronically ill elderly patients: the design of a randomised controlled trial [ISRCTN92331982]. BMC Public Health 2006;6(1):161.

16. Lorig KR, Sobel DS, Stewart AL, Brown BW, Jr., Bandura A, Ritter P, et al. Evidence suggesting that a chronic disease self-management program can improve health status while reducing hospitalization: a randomized trial. Med Care 1999;37(1):5-14.

17. Lorig KR, Holman H. Self-management education: history, definition, outcomes, and mechanisms. Ann Behav Med 2003;26(1):1-7.

18. Spitzer RL, Kroenke K, Williams JB. Validation and utility of a self-report version of PRIME-MD: the PHQ primary care study. Primary Care Evaluation of Mental Disorders. Patient Health Questionnaire. JAMA 1999;282(18):1737-44.

19. Sheehan DV, Lecrubier Y, Sheehan KH, Amorim P, Janavs J, Weiller E, et al. The Mini-International Neuropsychiatric Interview (M.I.N.I.): the development and validation of a structured diagnostic psychiatric interview for DSM-IV and ICD-10. J. Clin. Psychiatry 1998;59 Suppl 20:22-33.

20. Hamilton M. A rating scale for depression. J. Neurol. Neurosurg. Psychiatry1960;23:56-62. 


\section{CHAPTER 4}

21. Williams JW, Jr., Noel PH, Cordes JA, Ramirez G, Pignone M. Is this patient clinically depressed? JAMA 2002;287(9):1160-70.

22. Van Eijk JT, Diederiks JP, Kempen GI, Honig A, van der Meer K, Brenninkmeijer WJ. Development and feasibility of a nurse administered strategy on depression in community-dwelling patients with a chronic physical disease. Patient Educ. Couns. 2004;54(1):87-94.

23. Jonkers CCM, Lamers F, Bosma H, Metsemakers JF, Kempen GIJM, van Eijk JTM. Process evaluation of a minimal psychological intervention to reduce depression in chronically ill elderly persons. Patient Educ. Couns. 2007;68(3):252-257.

24. Geijer RMM, van Schayk CP, van Weel C, Sachs APE, van der Zwan ACC, Bottema BJAM, et al. NHGStandaard COPD en Astma bij Volwassenen: Behandeling [NHG Practice Guideline COPD: Treatment]. Huisarts Wet 1997;40:430-442.

25. Geijer RMM, Thiadens HA, Smeele IJM, Sachs APE, Bottema BJAM, van Hensbergen W, et al. NHGStandaard COPD en Astma bij Volwassenen: Diagnostiek [NHG Practice Guideline COPD: Diagnosis]. Huisarts Wet 2001;44(3):107-117.

26. Rutten GEHM, De Grauw WJC, Nijpels G, Goudswaard AN, Uitewaal PJM, Van der Does FEE, et al. NHGStandaard Diabetes mellitus type 2 [NHG Practice Guideline Diabetes mellitus type 2]. Huisarts Wet 2006;49(3):137-152.

27. Beck AT, Steer RA, Garbin MG. Psychometric properties of the Beck Depression Inventory: Twenty-five years of evaluation. Clinical Psychology Rev. 1988;8(1):77-100.

28. Beck AT, Ward CH, Mendelson M, Mock J, Erbaugh J. An inventory for measuring depression. Arch. Gen. Psychiatry 1961;4:561-571.

29. Depression Guideline Panel. Depression in Primary Care: volume 2. Treatment of Major Depression. Clinical Practice Guideline, number 5. Rockwille, M.D.: U.S. Department of Health and Human Services, Public Health Service, Agency for Health Care Policy and Research. AHCPR publication no. 93-0551, 1993.

30. Aaronson NK, Muller M, Cohen PD, Essink-Bot ML, Fekkes M, Sanderman R, et al. Translation, validation, and norming of the Dutch language version of the SF-36 Health Survey in community and chronic disease populations. J Clin Epidemio/1998;51(11):1055-68.

31. Pocock S. Clinical trials: A Practical Approach. Chichester: John Wiley and Sons, 1983.

32. Hotopf M. The pragmatic randomised controlled trial. Adv Psychiatr Treat 2002;8(5):326-333.

33. Beekman AT, Geerlings SW, Deeg DJ, Smit JH, Schoevers RS, de Beurs E, et al. The natural history of latelife depression: a 6-year prospective study in the community. Arch Gen Psychiatry 2002;59(7):605-11.

34. Cohen J. Statistical power analysis for the behavioral sciences. 2nd ed. Hillsdale, NJ: Lawrence Erlbaum Associates, 1988.

35. Hedlund M, Stalenheim G, Ekselius L, Carlsson M. Diagnostic agreement between a doctor and a nurse for psychiatric disorders: a pilot study. Nord J Psychiatry 2005;59(5):339-42. 


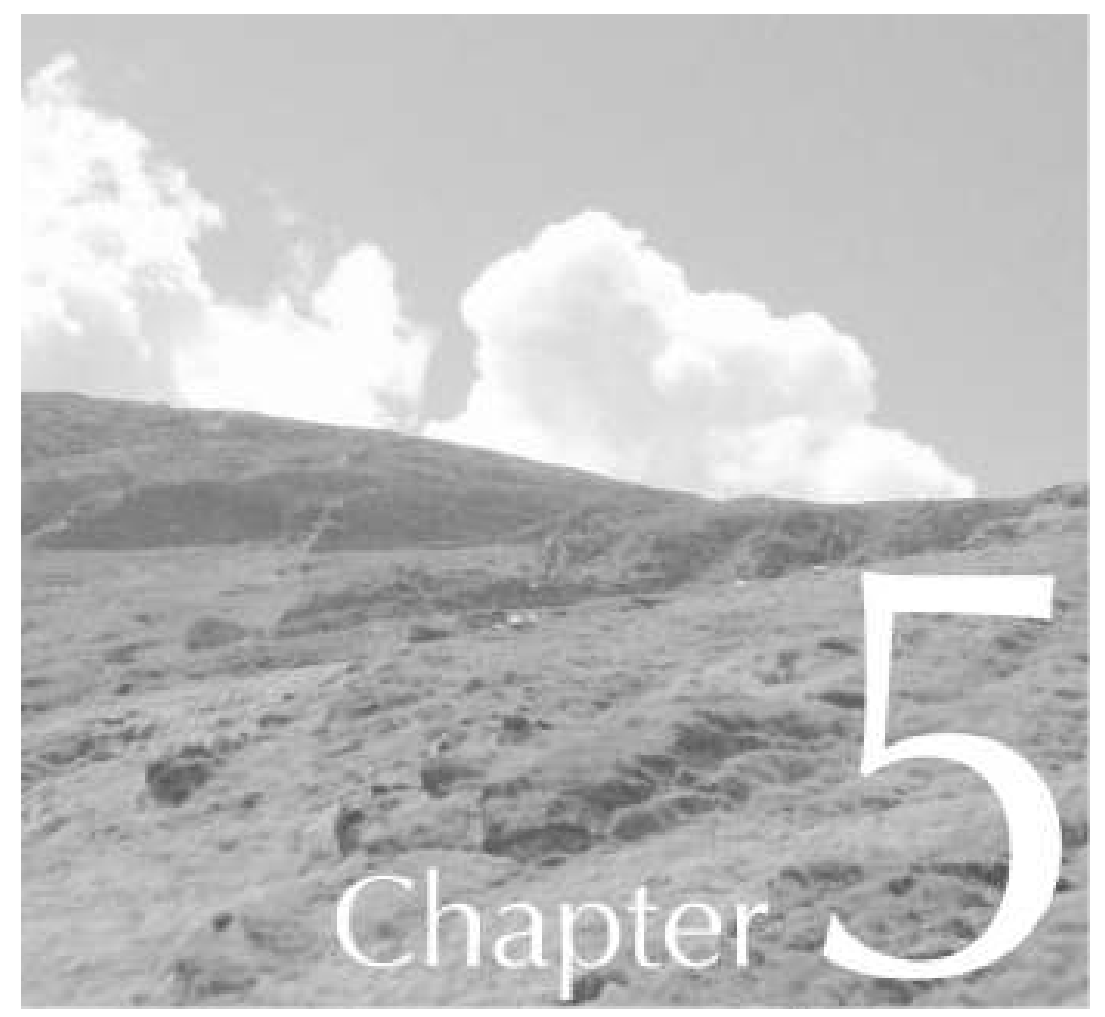

Treating depression in diabetes patients: does a minimal psychological intervention affect diabetesspecific quality of life and glycemic control? A randomised controlled trial 
CHAPTER 5 


\section{ABSTRACT}

Objective: To determine whether depression treatment with a nurse-led minimal psychological intervention (MPI) improves diabetes-specific quality of life and glycemic control in elderly diabetes patients.

Research Design and Methods: A pragmatic, randomised controlled trial was carried out comparing the MPI with usual care among 208 primary care patients of $\geq 60$ years with type 2 diabetes and co-occurring minor to moderate depression. Assessment of diabetes-specific quality of life (symptom and emotional distress) was made at baseline, one week, and three and nine months after treatment. HbA1c levels were obtained from the records of general practices.

Results: Only in higher-educated patients did the intervention have significant effect on both emotional and symptom distress. Further, we found an effect on symptom distress in men, and on emotional distress in patients with a shorter diabetes duration ( $<7$ years). A significant trend over time for HbA1c was found in favor of the intervention, with a significant difference in $\mathrm{HbA1c}$ after nine months.

Conclusions: The intervention had limited effects on diabetes-specific quality of life. As only certain subgroups benefited (more highly educated persons, men, persons with DM durations of $<7$ years), ways of increasing effectiveness in these groups should be explored. The potentially beneficial effect on glycemic control needs further research.

Trial registration: isrctn.org; identifier: ISRCTN92331982 
CHAPTER 5 
Approximately 10 to $17 \%$ of all diabetes patients suffer from depression. ${ }^{12}$ Depression is associated with lower quality of life, ${ }^{34}$ non-adherence to medical regimens and selfcare behaviours, ${ }^{5}$ DM complications, ${ }^{6}$ and higher medical costs and health care utilization. ${ }^{7}$ Furthermore, depression has a negative impact on physical activity, ${ }^{5}$ and may reduce glycemic control through central nervous system regulatory changes. ${ }^{3}$ Diabetes itself may negatively influence depression as poor glycemic control can induce negative moods, ${ }^{3}$ and physical illness is thought to be a predictor of poor depression outcome. ${ }^{8}$ Further, diabetes patients with co-occurring depression report more diabetes symptoms than those without. $^{9}$ Given these reciprocal negative consequences, depression treatment is of vital importance to diabetes patients; it may improve self-care behaviours and thus help reduce the burden of diabetes in terms of diabetes-specific symptom and emotional distress. ${ }^{10}$ It may also improve glycemic control, as was found in a small-scale trial evaluating CBT. ${ }^{11}$

We have previously shown that a nurse-led minimal psychological intervention (MPI) based on Cognitive Behavioral Therapy (CBT) and self-management principles reduced depressive symptoms and improved generic quality of life in elderly diabetes patients with co-occurring depression. ${ }^{12}$ In this paper, we evaluate whether the intervention also improves disease-specific quality of life and glycemic control. We expected that diabetes-specific quality of life in elderly diabetes patients with cooccurring minor or mild to moderate major depression would improve, and that intervention patients would have better glycemic control than control patients.

\section{RESEARCH DESIGN AND METHODS}

We conducted a pragmatic randomised controlled trial approved by the Medical Ethics Committee of the Maastricht University/ University Hospital Maastricht. Details of the study protocol have been described elsewhere. ${ }^{12} 13$ Briefly, between October 2003 and May 2005, patients were recruited from 89 primary care practices in the south of the Netherlands. First, all type 2 diabetes patients aged 60 years and over who did not meet the exclusion criteria were sent a depression screening questionnaire (Patient Health Questionnaire-9; PHQ-9). ${ }^{14}$ All patients who reported a) having at least two symptoms for more than half the time and b) one of these symptoms being loss of interest or depressed mood were invited to a diagnostic interview ( $n=534)$. A trained nurse interviewed the patient at home using the Mini International Neuropsychiatric Interview (MINI) ${ }^{15}$ and the Hamilton Depression Rating Scale (HDRS). ${ }^{16}$ All patients with minor depression or mild to moderate major depression were invited to participate in the study. Patients with severe major depression (HDRS>18) or suicidal risk were referred to their general practitioner (GP). In total, 208 patients with type 2 diabetes and minor depression or mild to moderate major depression signed informed consent forms and completed a baseline questionnaire (Figure 1). Randomisation was then performed, blinded for the researchers, by an external agency using a 
computerized random number generator with a block randomisation scheme stratified by general practice (block size of two).

\section{Intervention and usual care}

Patients allocated to the intervention group received the MPI at home in addition to usual care. The intervention is a nurse-administered, minimal psychological intervention, consisting of elements of CBT and self-management. Its aim was to educate patients to take responsibility for the daily management of their own illness and its consequences. A detailed description of the intervention has been published elsewhere. ${ }^{13} 17$ Nurses were trained by a GP, a psychologist, and a psychiatrist, and had regular booster sessions with the latter pair. The intervention is tailor-made: the number of visits depends upon progress. On average, patients had four intervention sessions of approximately one hour. A thorough process evaluation showed that the nurses adhered closely to protocol guidelines. ${ }^{18}$ Patients allocated to usual care received regular treatment according to the practice guidelines of the Dutch College of General Practitioners for type 2 diabetes. These guidelines include regular checkups for medical symptoms, but do not involve the detection and treatment of depressive symptoms. ${ }^{19}$ Co-interventions such as pharmacological depression treatments were allowed, and considered non-differential between groups. GPs were informed about which patients had participated in the trial only after the follow-up.

\section{Data collection}

Information regarding age, sex and educational level was collected at the time of the PHQ-9 screening. Diabetes duration and use of insulin and oral hypoglycemic agents were assessed in the baseline questionnaire.

Disease-specific quality of life was operationalized as diabetes-specific symptom distress assessed with the Diabetes Symptom Checklist - Revised (DSC-R), ${ }^{20}$ and emotional distress using the Problem Areas in Diabetes (PAID) questionnaire. ${ }^{21}$ Data were collected at baseline, one week and three and nine months after the treatment period. The PAID provides one total score; the DSC-R consists of a total score and subscores for eight dimensions: hyperglycemia, hypoglycemia, neuropathic pain, sensibility, fatigue, cognitive distress, cardiovascular symptoms, and ophthalmologic symptoms. Missing item scores on the DSC-R and PAID were imputed with the patient's mean item score if at least $50 \%$ of the items had been completed. The number of patients for whom imputation was not possible ranged from 2 to 12 for the DSC-R and 3 to 12 for the PAID per measurement and was evenly distributed per group.

After the follow-up period, all general practices were contacted in order to retrieve participants' hemoglobin A1c (HbA1c) data determined between the inclusion phase and the end of the follow-up (response: $94 \%$ ). Also, the medical records of patients 
living within the Maastricht area were checked for HbA1c data at the University Hospital Maastricht. In total, we retrieved HbA1c data for 135 patients.

\section{Statistical analysis}

In the total DELTA study population (of which the 208 diabetes patients were a subgroup), the power calculation was based on improvement in depressive symptoms and has been described elsewhere. ${ }^{12}$ Based on an $\alpha=0.05$ and $\beta=0.9,2 \times 103$ patients are sufficient to detect a minimum clinically relevant difference of 0.72 on the DSC-R total score, 9.03 on the PAID and $0.59 \%$ for HbA1c.

All analyses were conducted according to the intention-to-treat principle. Groups were checked for baseline comparability to identify potential confounders using Chisquare and t-tests. Mixed-effect, repeated measures ANCOVA models were used to test the differences in DSC-R and PAID scores between groups at follow-up. Age, sex, educational level, treatment group, baseline value of outcome, time, and the product term of time and group were standard inclusions in the model, and diabetes medication and duration were added as they were found to confound the relation between groups and outcome $(\geq 10 \%$ change in the estimate of group when added to the standard model). Furthermore, for significant differences, effect sizes (d) were calculated by dividing the difference in mean group scores by the pooled standard deviation. ${ }^{22}$ Additional per protocol analyses were done for the DSC-R and PAID, including patients who had received a complete intervention (i.e., all core steps of the intervention had been delivered, based on data from the process evaluation, $n=67)^{18}$ and excluding six control patients who responded positively to a contamination check during data collection. Further, last observation carried forward (LOCF) analyses were done. Possible influence from outliers was checked, but not observed. For HbA1c, we ran two models: one with the time of the HbA1c measurements on a continuous scale, and one with their time matched to that of the follow-up measurements (baseline, one week, three and nine months after treatment). This was done on the condition that the HbA1c was determined within a six-week period around the follow-up measurement. Data from 70 patients 37 control and 33 intervention patients) who had both a baseline HbA1c and $\geq 1$ follow-up measurements were used in analyses. The comparability of patients with and without HbA1c data available was checked and mixed-effect, repeated measures ANCOVA models were used (corrected for age, sex, educational level, baseline $\mathrm{HbA1c}$ level and time). 


\section{CHAPTER 5}

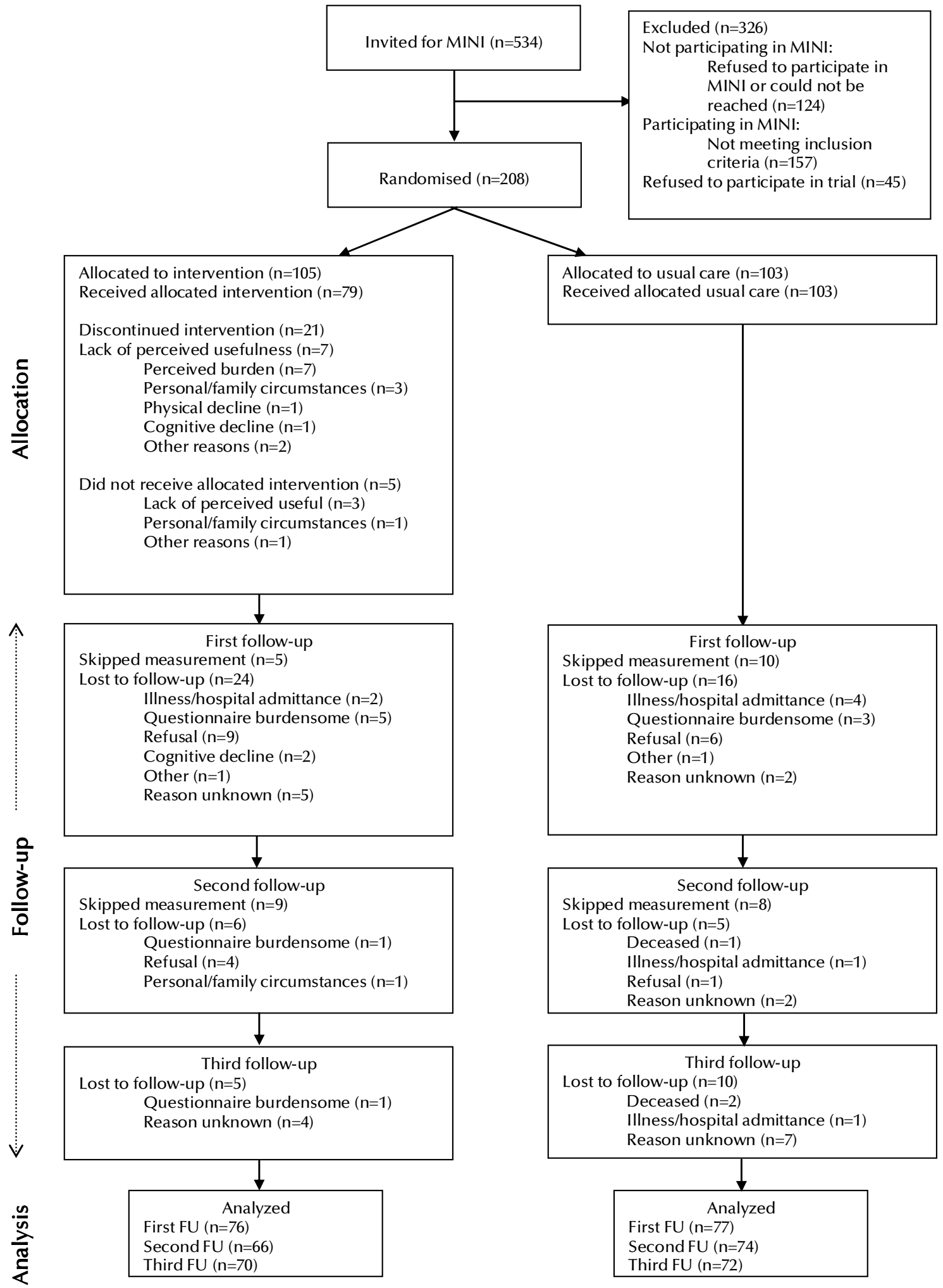

Figure 1 Flowchart 


\section{RESULTS}

Figure 1 shows patient flow within the study. The 45 eligible patients who chose not to participate were significantly older than those who did. The dropout percentage throughout the follow-up was comparable between the intervention and control groups (33\% vs. $30 \%, p=0.62)$. Dropout was associated only with higher age, with dropout characteristics not differing significantly between the intervention and control groups. Table 1 shows the baseline comparability of the groups. There were no significant differences at baseline.

The intervention's effects on quality of life in terms of symptom distress were limited (Table 2). Only at second follow-up did we find significant effects on the ophthalmologic symptom subscale (group difference $0.83, p<0.01$ ), on the cognitive stress subscale (group difference $0.72, \mathrm{p}=0.03$ ), and on the total DSC-R score (group difference $0.57, \mathrm{p}<0.01$ ). Effect sizes were medium: 0.45 for the ophthalmologic symptom subscale and total DSC-R score, and 0.38 for the cognitive stress subscale. For quality of life in terms of emotional distress (PAID), we found a consistent pattern in favor of the intervention, with a marginal significant difference after nine months (group difference 4.4, $\mathrm{p}=0.06$ ). Per protocol and LOCF analyses yielded similar results.

Patients for whom HbA1c values were available at baseline and at least one follow-up measurement $(n=70)$ were more often female, and used insulin and insulin plus oral hypoglycemic agents significantly more often than patients without available HbA1c data (data not shown). This latter finding is not surprising as, according to clinical guidelines, HbA1c should normally be tested once a year, but in patients using insulin two to four times a day, it should be tested every three to six months ${ }^{19}$. The intervention patients showed an HbA1c decline over time, whereas the control patients experienced an increase. The difference between groups after nine months was statistically significant in favor of the intervention (group difference $0.5 \%, p=0.02$ ). An additional analysis including the 70 patients with a baseline value, all available $\mathrm{HbA1c}$ observations (unmatched to the follow-up measurements) and time as a continuous variable further showed that the HbA1c trend over time differed significantly between groups $(p<0.00)$. 


\section{CHAPTER 5}

Table 1 Comparability of intervention and control groups for socio-demographic and disease-related variables and baseline values of outcomes $(n=208)$

\begin{tabular}{|c|c|c|c|c|}
\hline Variable & & $\begin{array}{c}\text { Usual care } \\
\mathrm{n}=103\end{array}$ & $\begin{array}{c}\text { MPI } \\
\mathrm{n}=105\end{array}$ & P-value \\
\hline \multicolumn{5}{|l|}{ Demographics } \\
\hline Age, yrs (SD) & & $69.7(6.6)$ & $70.7(6.6)$ & 0.25 \\
\hline \multicolumn{5}{|l|}{ Sex, no. $(\%)$} \\
\hline & Male & $51(49.5)$ & $51(48.6)$ & \\
\hline & Female & $52(50.5)$ & $54(51.4)$ & 0.89 \\
\hline \multicolumn{5}{|c|}{ Education level ${ }^{*+}$, no. $(\%)$} \\
\hline & Low & $34(33.3)$ & $35(34.0)$ & \\
\hline & Medium & $26(25.5)$ & $26(25.2)$ & \\
\hline & High & $42(41.2)$ & $42(40.8)$ & 0.56 \\
\hline
\end{tabular}

Disease-related variables

Diabetes duration, yrs (SD)

Medication $^{\ddagger}$, no. $(\%)$

None

Oral hypoglycemic agents

Insulin \& oral hypoglycemic agents

Insulin use

$\begin{array}{lll}9.8(9.1) & 8.2(8.8) & 0.22 \\ 17(16.8) & 14(14.3) & \\ 47(46.5) & 60(61.2) & \\ 21(20.8) & 14(14.3) & \\ 16(15.8) & 10(10.2) & 0.20\end{array}$

Baseline values of outcomes

DSC-R total, mean (SD)

$2.8(1.6) \quad 2.9(1.6) \quad 0.88$

DSC-R subscales, mean (SD)

Hyperglycemic

$3.5(2.8)$

$(2.9(1.6)$

0.88

Hypoglycemic

$2.3(2.1)$

$3.4(2.5)$

0.88

Polyneuropathic pain

$2.3(2.5)$

$2.3(2.1)$

0.94

Polyneuropathic sensory

$2.4(2.4)$

$2.1(2.5)$

0.65

Fatigue

$5.3(2.3)$

2.4 (2.5)

0.88

Cognitive stress

$3.2(2.4)$

$5.1(2.4)$

0.52

Cardiovascular

$2.4(2.1)$

$3.0(2.3)$

0.63

Ophthalmologic symptoms

$2.0(2.3)$

$2.6(2.0)$

0.62

$2.1(1.9) \quad 0.58$

PAID, mean (SD)

$23.4(19.5)$

$22.6(20.5)$

0.77

$\mathrm{HbA1c}^{\S}$, mean (SD)

$7.2(1.4)$

7.5 (1.2)

0.36

\footnotetext{
Abbreviations: $S D$, standard deviation; DSC-R, Diabetes Symptom Checklist - Revised; PAID, Problem Areas in Diabetes. DSC-R range is 0-10, with O being most favorable outcome; PAID range is 0-100, with 0 being the most favorable outcome.

* Low refers to primary school only, medium refers to lower vocational training or lower general education, high refers to higher vocational training, secondary school, higher professional education and university training

${ }^{+}$data missing from 3 patients

${ }^{*}$ data missing from 9 patients

${ }^{s}$ based on data from 37 control patients and 33 intervention patients
} 
EFFECTIVENESS IN DIABETES

Table 2 Diabetes-specific quality of life and glycemic control

\begin{tabular}{|c|c|c|c|c|c|c|}
\hline & Usual care & & MPI & & & Mean difference \\
\hline & Mean $(\mathrm{SE})^{*}$ & $\mathrm{~N}$ & Mean $(\mathrm{SE})^{*}$ & $\mathrm{~N}$ & $\begin{array}{c}\mathrm{P}- \\
\text { value }\end{array}$ & Mean $(95 \% \mathrm{Cl})^{*}$ \\
\hline \multicolumn{7}{|l|}{ Symptom distress } \\
\hline \multicolumn{7}{|l|}{ DSC-R Total } \\
\hline After 1 week & $2.88(0.14)$ & 70 & $2.84(0.16)$ & 65 & 0.82 & $0.05(-0.34$ to 0.43$)$ \\
\hline After 3 months & $3.24(0.15)$ & 69 & $2.67(0.17)$ & 59 & 0.006 & 0.57 (0.16 to 0.98$)$ \\
\hline After 9 months & $2.71(0.17)$ & 60 & $2.75(0.18)$ & 59 & 0.85 & $-0.05(-0.51$ to 0.42$)$ \\
\hline \multicolumn{7}{|l|}{ DSC-R Hyperglycemia } \\
\hline After 1 week & $3.17(0.25)$ & 71 & $3.64(0.27)$ & 64 & 0.17 & $-0.48(-1.16$ to 0.20$)$ \\
\hline After 3 months & $3.66(0.25)$ & 68 & $3.43(0.28)$ & 60 & 0.50 & $0.24(-0.46$ to 0.93$)$ \\
\hline After 9 months & $2.88(0.27)$ & 61 & $3.39(0.27)$ & 63 & 0.15 & $-0.51(-1.21$ to 0.19$)$ \\
\hline \multicolumn{7}{|l|}{ DSC-R Hypoglycemia } \\
\hline After 1 week & $2.20(0.22)$ & 69 & $2.42(0.23)$ & 65 & 0.47 & $-0.22(-0.81$ to 0.37$)$ \\
\hline After 3 months & $2.50(0.22)$ & 65 & $2.17(0.24)$ & 60 & 0.28 & $0.34(-0.27$ to 0.94$)$ \\
\hline After 9 months & $2.05(0.23)$ & 60 & $1.85(0.24)$ & 58 & 0.52 & $0.20(-0.42$ to 0.2$)$ \\
\hline \multicolumn{7}{|l|}{ DSC-R Pain } \\
\hline After 1 week & $2.54(0.25)$ & 69 & $2.52(0.27)$ & 66 & 0.96 & $0.02(-0.66$ to 0.69$)$ \\
\hline After 3 months & $2.91(0.25)$ & 67 & $2.68(0.27)$ & 61 & 0.50 & $0.24(-0.45$ to 0.92$)$ \\
\hline After 9 months & $2.29(0.26)$ & 59 & $2.33(0.27)$ & 62 & 0.92 & $-0.04(-0.74$ to 0.66$)$ \\
\hline \multicolumn{7}{|l|}{ DSC-R Sensory } \\
\hline After 1 week & $2.33(0.21)$ & 68 & $2.58(0.22)$ & 66 & 0.37 & $-0.26(-0.82$ to 0.31$)$ \\
\hline After 3 months & $2.92(0.22)$ & 67 & $2.43(0.24)$ & 61 & 0.11 & $0.48(-0.11$ to 1.08$)$ \\
\hline After 9 months & $2.18(0.18)$ & 59 & $2.35(0.26)$ & 63 & 0.62 & $-0.17(-0.85$ to 0.51$)$ \\
\hline \multicolumn{7}{|l|}{ DSC-R Fatigue } \\
\hline After 1 week & $5.00(0.21)$ & 68 & $4.83(0.23)$ & 65 & 0.57 & $0.16(-0.40$ to 0.72$)$ \\
\hline After 3 months & $4.91(0.22)$ & 67 & $4.65(0.24)$ & 59 & 0.40 & $0.26(-0.34$ to 0.86$)$ \\
\hline After 9 months & $4.89(0.26)$ & 58 & $4.94(0.28)$ & 60 & 0.87 & $-0.06(-0.77$ to 0.65$)$ \\
\hline \multicolumn{7}{|l|}{ DSC-R Cognitive stress } \\
\hline After 1 week & $3.35(0.23)$ & 70 & $3.28(0.25)$ & 65 & 0.84 & $0.07(-0.56$ to 0.69$)$ \\
\hline After 3 months & $3.63(0.23)$ & 67 & $2.91(0.25)$ & 60 & 0.03 & 0.72 (0.08 to 1.36$)$ \\
\hline After 9 months & $3.16(0.24)$ & 60 & $2.98(0.25)$ & 58 & 0.58 & $0.18(-0.46$ to 0.83$)$ \\
\hline \multicolumn{7}{|l|}{ DSC-R Cardiovascular } \\
\hline After 1 week & $2.74(0.19)$ & 68 & $2.84(0.21)$ & 64 & 0.72 & $-0.10(-0.61$ to 0.42$)$ \\
\hline After 3 months & $3.07(0.19)$ & 62 & $2.72(0.22)$ & 59 & 0.22 & $0.34(-0.20$ to 0.89$)$ \\
\hline After 9 months & $2.53(0.22)$ & 62 & $2.74(0.24)$ & 58 & 0.50 & $-0.21(-0.83$ to 0.41$)$ \\
\hline \multicolumn{7}{|l|}{ DSC-R Ophthalmologic } \\
\hline After 1 week & $2.40(0.22)$ & 69 & $1.86(0.24)$ & 64 & 0.07 & $0.54(-0.05$ to 1.14$)$ \\
\hline After 3 months & $2.52(0.22)$ & 69 & $1.69(0.25)$ & 58 & 0.008 & 0.83 (0.22 to 1.43$)$ \\
\hline After 9 months & $2.22(0.23)$ & 61 & $1.96(0.24)$ & 57 & 0.40 & $0.27(-0.35$ to 0.88$)$ \\
\hline
\end{tabular}




\begin{tabular}{|c|c|c|c|c|c|c|}
\hline & Usual care & & $\mathrm{MPI}$ & & & $\begin{array}{l}\text { Mean difference } \\
\text { (Usual care - MPI) }\end{array}$ \\
\hline & Mean (SE)* & $\mathrm{N}$ & Mean $(\mathrm{SE})^{*}$ & $\mathrm{~N}$ & $\begin{array}{c}\text { P- } \\
\text { value }\end{array}$ & Mean $(95 \% \mathrm{Cl}) *$ \\
\hline \multicolumn{7}{|l|}{ Emotional distress } \\
\hline \multicolumn{7}{|l|}{ PAID } \\
\hline After 1 week & $24.00(1.62)$ & 71 & $23.42(1.73)$ & 67 & 0.80 & $0.59(-3.80$ to 4.96$)$ \\
\hline After 3 months & $23.56(1.63)$ & 69 & $21.49(1.79)$ & 60 & 0.37 & $2.07(-2.44$ to 6.58$)$ \\
\hline After 9 months & $22.89(1.72)$ & 61 & $18.49(1.76)$ & 62 & 0.06 & $4.40(-0.16$ to 8.97$)$ \\
\hline \multicolumn{7}{|l|}{ Glycemic control } \\
\hline \multicolumn{7}{|l|}{ HbA1c } \\
\hline After 1 week & $7.4(0.2)$ & 18 & $7.8(0.2)$ & 17 & 0.09 & $-0.4(-0.9$ to 0.1$)$ \\
\hline After 3 months & $7.5(0.2)$ & 15 & $7.4(0.2)$ & 12 & 0.81 & $0.1(-0.5$ to 0.6$)$ \\
\hline After 9 months & $7.8(0.2)$ & 17 & $7.3(0.2)$ & 20 & 0.02 & 0.5 (0.1 to 1.0$)$ \\
\hline
\end{tabular}

Abbreviations: SE, standard error; $95 \% C l$, 95\% confidence interval; DSC-R, Diabetes Symptom Checklist - Revised; PAID, Problem Areas in Diabetes. DSC-R range is 0-10, with O being most favorable outcome; $P A I D$ range is $0-100$, with 0 being the most favorable outcome.

*estimates from mixed-model regression analyses, corrected for age, sex, education level, diabetes medication and duration, and baseline value of outcome measure. HbA1c models corrected for age, sex, education level, and baseline value of HbA1c.

As the intervention seemed to have little effect on symptom and emotional distress, we performed post-hoc analyses for the DSC-R total score and PAID to evaluate the effectiveness within subgroups. To the model, we added interaction terms per group of sex, age, education (tertiles), medication use and diabetes duration to the model. Where interaction terms were significant $(p<0.10)$, stratified subgroup analyses were done. These additional analyses revealed that the intervention did have significant effects on symptom distress (DSC-R total score) on all follow-up measurements in male patients (group difference after nine months $0.7, p=0.01, d=0.99$ ), but not in female patients (groups difference after nine months $-0.9, p=0.01, d=-0.66$ ). Furthermore, in patients with a diabetes duration of $<7$ years, the intervention group on average had more favorable scores for emotional distress (PAID) than the control group at all follow-up measurements, with a significant effect at second follow-up (group difference 6.6, $\mathrm{p}=0.04, \mathrm{~d}=0.39$ ). In patients with a diabetes duration of $\geq 7$ years, the intervention group scored more favorably only at the last follow-up. Distinct differences were also found for education on both symptom and emotional distress. In the lowest education tertile, intervention patients on average had worse outcomes than control patients, with effect sizes of -0.67 on the DSC-R and -0.65 on the PAID. In the middle and highest education tertiles, intervention patients benefited from the intervention and scored significantly higher than the control patients (Table 3). Effect sizes were large on the DSC-R; 1.22 for the middle tertile and 0.83-0.93 for the highest tertile. On the PAID, the effect size for the middle tertile was 0.77 and for the highest tertile 0.56 . 
Table 3 Diabetes-specific quality of life outcomes stratified by education level.

\begin{tabular}{|c|c|c|c|c|c|c|}
\hline & \multicolumn{2}{|c|}{ Usual care } & \multicolumn{2}{|l|}{ MPI } & & \multirow{2}{*}{$\begin{array}{c}\text { Mean difference } \\
\text { (Usual care-MPI) }\end{array}$} \\
\hline & Mean $(\mathrm{SE})^{*}$ & $\mathrm{~N}$ & Mean (SE)* & $\mathrm{N}$ & P-value & \\
\hline \multicolumn{7}{|l|}{ DSC-R Total } \\
\hline \multicolumn{7}{|l|}{ Low } \\
\hline After 1 week & $3.04(0.32$ & 22 & $3.56(0.35)$ & 20 & 0.24 & $-0.52(-1.42$ to 0.37$)$ \\
\hline After 3 months & $3.31(0.32$ & 24 & $3.36(0.38)$ & 15 & 0.92 & $-0.05(-.97$ to 0.88$)$ \\
\hline After 9 months & $2.42(0.34$ & 20 & $3.41(0.39)$ & 15 & $<0.05$ & $-0.99(-1.98$ to -0.002$)$ \\
\hline \multicolumn{7}{|l|}{ Medium } \\
\hline After 1 week & $2.93(0.21)$ & 16 & $2.42(0.22)$ & 17 & 0.08 & $0.50(-0.05$ to 1.05$)$ \\
\hline After 3 months & $3.51(0.22)$ & 17 & $2.41(0.24)$ & 16 & $<0.00$ & 1.10 (0.51 to 1.70$)$ \\
\hline After 9 months & $2.58(0.30)$ & 14 & $2.94(0.29)$ & 17 & 0.38 & $-0.36(-1.17$ to 0.46$)$ \\
\hline \multicolumn{7}{|l|}{ High } \\
\hline After 1 week & $2.91(0.18)$ & 32 & $2.59(0.19)$ & 28 & 0.19 & $0.33(-0.16$ to 0.81$)$ \\
\hline After 3 months & $3.19(0.19)$ & 28 & $2.38(0.20)$ & 28 & 0.002 & $0.81(0.30$ to 1.31$)$ \\
\hline After 9 months & $3.17(0.21)$ & 26 & $2.20(0.20)$ & 27 & 0.001 & $0.96(0.42$ to 1.51$)$ \\
\hline \multicolumn{7}{|l|}{ PAID } \\
\hline \multicolumn{7}{|l|}{ Low } \\
\hline After 1 week & $22.88(3.05)$ & 22 & $31.97(3.26)$ & 20 & 0.04 & $-9.09(-17.5$ to -0.67$)$ \\
\hline After 3 months & $19.71(2.95)$ & 24 & $23.52(3.62)$ & 15 & 0.40 & $-3.80(-12.63$ to 5.02$)$ \\
\hline After 9 months & $20.97(3.19)$ & 19 & $21.33(3.42)$ & 17 & 0.94 & $-0.36(-9.24$ to 8.52$)$ \\
\hline \multicolumn{7}{|l|}{ Medium } \\
\hline After 1 week & $27.10(3.59)$ & 16 & $17.92(3.54)$ & 17 & 0.05 & $9.18(-0.02$ to 18.38$)$ \\
\hline After 3 months & $28.92(3.51)$ & 17 & $18.07(3.56)$ & 17 & 0.02 & 10.85 (1.72 to 19.98$)$ \\
\hline After 9 months & $22.67(3.69)$ & 15 & $14.09(3.53)$ & 17 & 0.07 & $8.58(-0.68$ to 17.85$)$ \\
\hline \multicolumn{7}{|l|}{ High } \\
\hline After 1 week & $24.85(2.15)$ & 33 & $20.62(2.25)$ & 30 & 0.15 & $4.23(-1.52$ to 9.98$)$ \\
\hline After 3 months & $25.23(2.26)$ & 28 & $21.68(2.33)$ & 28 & 0.25 & 3.55 (-2.48 to 9.58$)$ \\
\hline After 9 months & $25.47(2.33)$ & 27 & $18.78(2.28)$ & 28 & 0.03 & 6.69 (0.62 to 12.76$)$ \\
\hline
\end{tabular}

Low refers to primary school only, medium refers to lower vocational training or lower general education, high refers to higher vocational training, secondary school, higher professional education and university training.

Abbreviations: SE, standard error; 95\% CI, 95\% confidence interval; DSC-R, Diabetes Symptom Checklist - revised; PAID, Problem Areas in Diabetes. DSC- $R$ range is $0-10$, with 0 being most favorable outcome; $P A I D$ range is $0-100$, with 0 being the most favorable outcome.

*estimates from mixed-model regression analyses, corrected for age, sex, education level, diabetes medication, duration of diabetes, and baseline value of outcome measure.

\section{CONCLUSION}

Overall, the nurse-led minimal psychological intervention had a moderate effect on disease-specific quality of life. Additional analyses revealed that it benefited symptom distress in men, but not in women, while emotional distress was more often improved in patients who had had diabetes for less than seven years. The most striking finding, however, was that patients within the lowest education tertile on average did not benefit from the intervention. Its effects on symptom and emotional distress were only found in the middle and highest education tertile, with medium to large effect sizes. Further, we found a difference in mean HbA1c level in favor of the intervention after nine months, with a significant difference between groups in trend over time. 
Several aspects should be noted here. First, patients who dropped out were older than those who completed all questionnaires, but dropout was evenly distributed between treatment groups. Second, HbA1c data was obtained from general practices, and although the data give a good indication of the treatment effect, the number of observations was limited. HbA1c measurements in all patients at fixed points during the data collection would have provided stronger evidence. Third, the clinical relevance of the findings of the DSC-R and PAID is difficult to interpret as there is no clear definition of a clinically relevant difference. Both scales are said to be responsive to change, but cut-off points for minimum clinically relevant differences are lacking. ${ }^{2023}$ Finally, post-hoc analyses should be interpreted with caution as significant findings may have been the result of multiple testing.

We found no effect on diabetes-specific quality of life in patients within the lowest education tertile. Previous studies evaluating the influence of socioeconomic status on response to MPI treatment also found diminished response in lower educated persons with respect to depressive symptoms. ${ }^{24}$ As patients with a lower socioeconomic status already are at higher risk for diabetes complications, ${ }^{25}$ it is important to identify the factors related to this diminished response and evaluate the extent to which adaptations can be made to the intervention to improve its effectiveness for this group. The factors involved may range from intellectual ability and amenability to change in the patients' cultural environment and perceived social support. Interestingly, Areán and colleagues found that lower-income elderly persons experienced similar benefits on depression outcomes to those of the higher-income elderly from depression care management and problem-solving therapy. However, ongoing care management of up to a year was recommended for lower-income persons, as their physical functioning improved more slowly than that of higher-income patients. ${ }^{26}$ These findings suggest that booster sessions or some other form of follow-up support in everyday self-management skills may improve outcomes for persons with lower socioeconomic status.

The intervention's effect on glycemic control is interesting, as most other studies have failed to find similar effects through depression treatment. Only a small trial by Lustman and colleagues showed that patients receiving CBT and education had significantly better glycemic control than those receiving education only. ${ }^{11}$ Two studies evaluating collaborative depression care for diabetes patients did not find effects on glycemic control despite depressive symptoms being reduced by enhanced care. $^{1027}$ Several smaller trials evaluating antidepressant treatment found either no effects ${ }^{2829}$ or adverse effects on glycemic control. ${ }^{30}$ However, whether a difference of $0.5 \%$ is clinically relevant is debatable, although the lower the $\mathrm{HbA} 1 \mathrm{c}$ is, the better. The suggestion that CBT-based depression treatments improve glycemic control is certainly worth further investigation on a larger scale with protocolized follow-up measurements.

To conclude, although the effectiveness of the nurse-led minimal psychological intervention in reducing depressive symptoms and improving generic quality of life has been previously established, this intervention only improved diabetes-specific 
quality of life in certain subgroups. As some patient groups did not benefit, ways of increasing the intervention's beneficial effects in these groups, such as adding followup meetings, should be explored. Likewise, the potentially beneficial effect of the MPI on glycemic control needs further examination.

\section{Acknowledgements}

We thank our nurses Kitty Daemen, Henny Geelen, Francine Hendriks, and Mieke Witte for administering the intervention with great dedication and enthusiasm. We are also grateful for the contributions of Wendy Engering to patient recruitment and Dorien Mintjes to data entry, and the Centre for Data and Information Management (MEMIC) for providing the data management system. This study was funded by the Netherlands Organisation for Health Research and Development (ZonMw) program on Health Care Efficiency Research (grant number 945-03-047). 


\section{CHAPTER 5}

\section{REFERENCES}

1. Anderson RJ, Freedland KE, Clouse RE, Lustman PJ. The prevalence of comorbid depression in adults with diabetes: a meta-analysis. Diabetes Care 2001;24(6):1069-78.

2. Ali S, Stone MA, Peters JL, Davies MJ, Khunti K. The prevalence of co-morbid depression in adults with Type 2 diabetes: a systematic review and meta-analysis. Diabet Med 2006;23(11):1165-73.

3. Lustman PJ, Clouse RE. Depression in diabetic patients: the relationship between mood and glycemic control. J Diabetes Complications 2005;19(2):113-22.

4. McCollum M, Ellis SL, Regensteiner JG, Zhang W, Sullivan PW. Minor depression and health status among US adults with diabetes mellitus. Am J Manag Care 2007;13(2):65-72.

5. Gonzalez JS, Safren SA, Cagliero E, Wexler DJ, Delahanty L, Wittenberg E, et al. Depression, self-care, and medication adherence in type 2 diabetes: relationships across the full range of symptom severity. Diabetes Care 2007;30(9):2222-7.

6. de Groot M, Anderson R, Freedland KE, Clouse RE, Lustman PJ. Association of depression and diabetes complications: a meta-analysis. Psychosom Med 2001;63(4):619-30.

7. Ciechanowski PS, Katon WJ, Russo JE. Depression and diabetes: impact of depressive symptoms on adherence, function, and costs. Arch Intern Med 2000;160(21):3278-85.

8. Cole MG, Bellavance F, Mansour A. Prognosis of depression in elderly community and primary care populations: a systematic review and meta-analysis. Am J Psychiatry 1999;156(8):1182-9.

9. Katon W, Lin EH, Kroenke K. The association of depression and anxiety with medical symptom burden in patients with chronic medical illness. Gen Hosp Psychiatry 2007;29(2):147-55.

10. Williams JW, Jr., Katon W, Lin EH, Noel PH, Worchel J, Cornell J, et al. The effectiveness of depression care management on diabetes-related outcomes in older patients. Ann Intern Med 2004;140(12):1015-24.

11. Lustman PJ, Griffith LS, Freedland KE, Kissel SS, Clouse RE. Cognitive behavior therapy for depression in type 2 diabetes mellitus. A randomized, controlled trial. Ann Intern Med 1998;129(8):613-21.

12. Lamers F, Jonkers CCM, Bosma H, Kempen GIJM, Meijer JAMJ, Penninx BWJH, et al. The effectiveness of a minimal psychological intervention in chronically ill elderly patients with depression: a randomised trial (the DELTA-study). submitted.

13. Lamers F, Jonkers CC, Bosma H, Diederiks JP, van Eijk JT. Effectiveness and cost-effectiveness of a minimal psychological intervention to reduce non-severe depression in chronically ill elderly patients: the design of a randomised controlled trial [ISRCTN92331982]. BMC Public Health 2006;6(1):161.

14. Spitzer RL, Kroenke K, Williams JB. Validation and utility of a self-report version of PRIME-MD: the PHQ primary care study. Primary Care Evaluation of Mental Disorders. Patient Health Questionnaire. JAMA 1999;282(18):1737-44.

15. Sheehan DV, Lecrubier Y, Sheehan $\mathrm{KH}$, Janavs J, Weiller E, Keskiner A, et al. The validity of the Mini International Neuropsychiatric Interview (MINI) according to the SCID-P and its reliability. Eur Psychiatry 1997;12(5):232-241.

16. Hamilton M. A rating scale for depression. J. Neurol. Neurosurg. Psychiatry 1960;23:56-62.

17. Van Eijk JT, Diederiks JP, Kempen GI, Honig A, van der Meer K, Brenninkmeijer WJ. Development and feasibility of a nurse administered strategy on depression in community-dwelling patients with a chronic physical disease. Patient Educ. Couns. 2004;54(1):87-94.

18. Jonkers CCM, Lamers F, Bosma H, Metsemakers JF, Kempen GIJM, van Eijk JTM. Process evaluation of a minimal psychological intervention to reduce depression in chronically ill elderly persons. Patient Educ. Couns. 2007;68(3):252-257.

19. Rutten GEHM, De Grauw WJC, Nijpels G, Goudswaard AN, Uitewaal PJM, Van der Does FEE, et al. NHGStandaard Diabetes mellitus type 2 [NHG Practice Guideline Diabetes mellitus type 2]. Huisarts Wet 2006;49(3):137-152.

20. Grootenhuis PA, Snoek FJ, Heine RJ, Bouter LM. Development of a type 2 diabetes symptom checklist: a measure of symptom severity. Diabet Med 1994;11(3):253-61.

21. Snoek FJ, Pouwer F, Welch GW, Polonsky WH. Diabetes-related emotional distress in Dutch and U.S. diabetic patients: cross-cultural validity of the problem areas in diabetes scale. Diabetes Care 2000;23(9):1305-9. 
22. Cohen J. Statistical power analysis for the behavioral sciences. 2nd ed. Hillsdale, NJ: Lawrence Erlbaum Associates, 1988.

23. Welch G, Weinger K, Anderson B, Polonsky WH. Responsiveness of the Problem Areas In Diabetes (PAID) questionnaire. Diabet Med 2003;20(1):69-72.

24. Bosma H, Lamers F, Jonkers CCM, van Eijk JTM. Self-management: the holy grail for all or for some only? A randomised controlled trial.

25. Larranaga I, Arteagoitia JM, Rodriguez JL, Gonzalez F, Esnaola S, Pinies JA. Socio-economic inequalities in the prevalence of Type 2 diabetes, cardiovascular risk factors and chronic diabetic complications in the Basque Country, Spain. Diabet Med 2005;22(8):1047-53.

26. Arean PA, Gum AM, Tang L, Unutzer J. Service use and outcomes among elderly persons with low incomes being treated for depression. Psychiatr Serv 2007;58(8):1057-64.

27. Katon WJ, Von Korff M, Lin EH, Simon G, Ludman E, Russo J, et al. The Pathways Study: a randomized trial of collaborative care in patients with diabetes and depression. Arch Gen Psychiatry 2004;61(10):1042-9.

28. Lustman PJ, Freedland KE, Griffith LS, Clouse RE. Fluoxetine for depression in diabetes: a randomized double-blind placebo-controlled trial. Diabetes Care 2000;23(5):618-23.

29. Paile-Hyvarinen M, Wahlbeck K, Eriksson JG. Quality of life and metabolic status in mildly depressed patients with type 2 diabetes treated with paroxetine: a double-blind randomised placebo controlled 6month trial. BMC Fam Pract 2007;8:34.

30. Lustman PJ, Griffith LS, Clouse RE, Freedland KE, Eisen SA, Rubin EH, et al. Effects of nortriptyline on depression and glycemic control in diabetes: results of a double-blind, placebo-controlled trial. Psychosom Med 1997;59(3):241-50. 
CHAPTER 5 


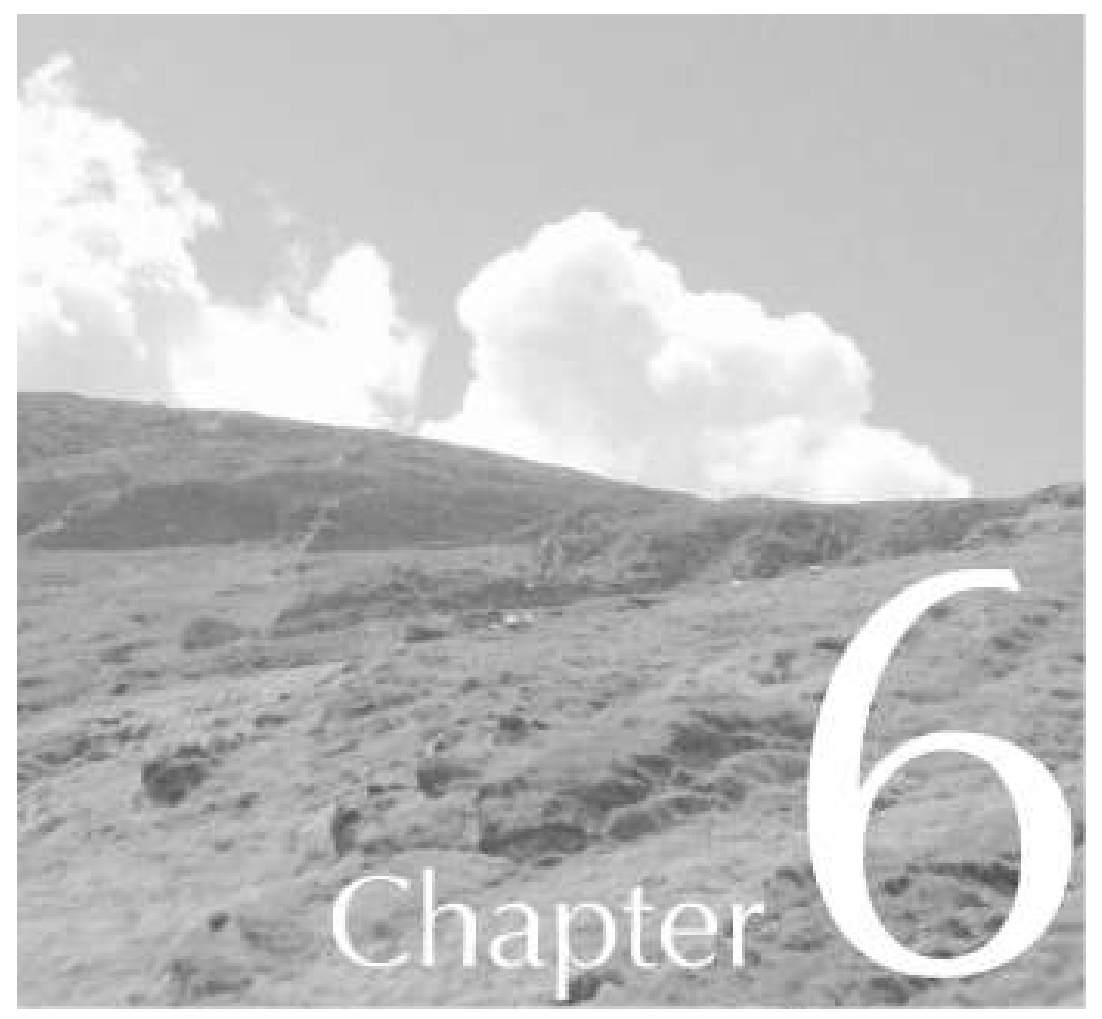

Improving quality of life in elderly COPD patients with minor to moderate depression: a randomised trial on the effectiveness of a minimal psychological intervention 
CHAPTER 6 


\section{ABSTRACT}

Background: Depression and anxiety are highly prevalent in elderly COPD patients. Since symptoms of depression and anxiety reduce quality of life in these patients, treatments aimed at improving mental health may improve their quality of life. Two advantages of psychological treatments are that they teach patients valuable and enduring skills, and that they can very well be administered by trained nurses. This study evaluated the effectiveness of a nurse-led Minimal Psychological Intervention (MPI) in reducing depression and anxiety, and improving disease-specific quality of life in elderly COPD patients.

Methods: In a randomised controlled trial that was part of the Depression in Elderly with Long-Term Afflictions (DELTA) study, an MPI was compared with usual care in COPD patients. COPD patients aged 60 years or over, and with minor or mild to moderate major depression were recruited in primary care $(n=187)$. The intervention was based on principles of cognitive behavioural therapy (CBT) and self-management. Outcomes were symptoms of depression (Beck Depression Inventory - BDI), symptoms of anxiety (Symptom Checklist - SCL), and disease-specific quality of life (Saint George's Respiratory Questionnaire -SGRQ), assessed at baseline and at one week and three and nine months after the intervention. Mixed model, repeated measures ANCOVA was used to analyse outcomes.

Results: Patients receiving the MPI had significantly fewer depressive symptoms (mean BDI difference 2.92, $\mathrm{p}=0.04$ ) and fewer symptoms of anxiety (mean SCL difference $3.69, p=0.003$ ) at nine months than patients receiving usual care. Further, mean SGRQ scores were significantly more favourable in the intervention group than in the control group after nine months (mean SGRQ difference 7.94, $\mathrm{p}=0.004$ ).

Conclusion: Our nurse-led MPI reduced symptoms of depression and anxiety and improved disease-specific quality of life in elderly COPD patients. The MPI appears to be a valuable addition to existing disease-management programmes for COPD patients. 
CHAPTER 6 


\section{INTRODUCTION}

Chronic obstructive pulmonary disease (COPD) is a prevalent disease in elderly persons. ${ }^{1}$ Since COPD is irreversible, disabling and progressive, treatment aims to prevent deterioration of the disease and maintain a high quality of life.

Depression and anxiety are highly prevalent in elderly COPD patients, ${ }^{23}$ and both are known to reduce quality of life. ${ }^{4-6}$ Since COPD is characterized by systemic inflammation, ${ }^{7}$ and depression is known to have an adverse influence on immunologic processes, ${ }^{89}$ co-occurrence of COPD and depression may lead to further deterioration of patients' health status due to interaction. Breaking through this downward spiral is thus an important step towards improving quality of life. In view of the relationship between quality of life and depression and anxiety, treatments to reduce depression and anxiety in COPD patients may also improve their quality of life. ${ }^{310}$

Depression and anxiety can be treated with antidepressants, ${ }^{31112}$ although some claim that the evidence for their effectiveness in older COPD patients is sparse and inconclusive. ${ }^{11} 13$ Furthermore, treatment with antidepressants may be complicated by problems like medication side effects or refusal of treatment by COPD patients. ${ }^{11} 13$ Psychological and self-management interventions may be better alternatives, especially as they teach patients valuable and enduring skills to cope with their disease, thus potentially ensuring a more lasting effect, extending beyond the end of treatment. Furthermore, these interventions can be administered by trained paramedical professionals, like nurses, complementing the care of physicians. ${ }^{14}{ }^{15}$ Few trials have assessed the effectiveness of psychological and self-management interventions in elderly COPD patients in terms of reducing symptoms of depression and anxiety. A recent systematic review based on four, relatively small, studies, found only limited evidence for the effectiveness of cognitive behavioural therapy (CBT) in reducing anxiety and depression in COPD patients, although the direction of treatment effects on both depression and anxiety scales mainly favoured $\mathrm{CBT} .{ }^{16} \mathrm{~A}$ recent review of selfmanagement education for COPD patients produced inconclusive results for the effect on the generic quality of life outcome, but found significant effects on a disease-specific quality of life measure. ${ }^{17}$ Both reviews recommend further research into CBT and self-management for COPD patients.

We have developed a nurse-administered minimal psychological intervention (MPI) based on the principles of CBT and self-management. The intervention has previously been evaluated in the Depression in Elderly with long-Term Afflictions (DELTA) study in a population of elderly patients with diabetes or COPD. ${ }^{18}$ The current contribution reports on the effect of the MPI on disease-specific quality of life and symptoms of depression and anxiety in the subgroup of elderly COPD patients with co-morbid depression. We hypothesized that our MPI would reduce symptoms of depression and anxiety and would also improve disease-specific quality of life. 


\section{MATERIALS AND METHODS}

\section{Study design}

Detailed information on the study protocol has been published elsewhere. ${ }^{1819}$ Briefly, a randomised controlled trial was conducted, with patients allocated to either the MPI or care as usual after signing informed consent. Self-administered questionnaires were used to collect data at baseline and at one week and three and nine months after the treatment period. Data entry was performed by researchers blinded for the allocation. Approval for this study was obtained from the Medical Ethics Committee of Maastricht University / University Hospital Maastricht.

\section{Participants and procedures}

Between October 2003 and May 2005, patients were recruited in 89 general practices in the south of the Dutch province of Limburg. We first selected all patients aged 60 years and older with an International Classification of Primary Care (ICPC) code for emphysema / COPD or chronic bronchitis. If ICPC codes were not available, lists of patients with repeat prescriptions for bronchodilatory drugs were obtained. The patients' general practitioners (GPs) then excluded from this initial broad selection all patients who did not have COPD, were bedridden, were on a waiting list for a nursing home, had major depression, used antidepressants, had major psychiatric conditions or were currently receiving psychosocial/psychiatric treatment, had serious cognitive problems, had recently lost their spouse or were not fluent in Dutch. All remaining patients aged 60 years and older who did have COPD according to their GP were sent a depression screening questionnaire (Patient Health Questionnaire-9 - PHQ-9). ${ }^{20}$ After the PHQ-9 screening, all patients who reported having at least two symptoms of depression, one being loss of interest or depressed mood, were invited to a diagnostic interview, consisting of the Mini International Neuropsychiatric Interview (MINI) ${ }^{21}$ and the Hamilton Depression Rating Scale (HDRS). ${ }^{22}$ The interview was conducted at the patients' home by a trained nurse. Patients with minor depression, mild major depression, moderate major depression or dysthymia were included in the study. Patients with severe major depression according to the HDRS (HDRS $>18$ ) and patients with suicidal risk were excluded and referred back to their GP. All COPD patients thus included ( $n=187)$ signed an informed consent form and were randomised. The researchers entered patients into a computer connected to an external agency, which performed the randomisation using a computerized random number generator. A block randomisation scheme was used, stratified for general practice. Block size was set at two, as we expected to include only a small number of patients per practice. 


\section{Intervention and usual care}

Patients allocated to the intervention group received the MPI at home, supplementary to usual care according to the clinical Guidelines for the Treatment of COPD of the Dutch College of General Practitioners. ${ }^{23}$ The intervention is a nurse-administered, minimal psychological intervention, consisting of elements of CBT and selfmanagement. Nurses were trained by a GP, a psychologist and a psychiatrist, and had regular booster sessions with the psychiatrist. The intervention was tailored to individual patients. Depending upon progress, patients received two to ten visits over a period of at most three months. Patients had on average four intervention contacts, each lasting approximately one hour. Table 1 lists the five phases of the intervention; a more detailed description of the intervention has been published elsewhere. ${ }^{1924}$ To ensure that the nurses adhered to the protocol during the study, they were asked to keep checklists, covering all essential intervention steps, for each patient. Examination of these lists showed that nurses had adhered closely to the guidelines in the protocol. ${ }^{25}$ Patients allocated to the control group received care as usual according to the above-mentioned clinical guidelines.

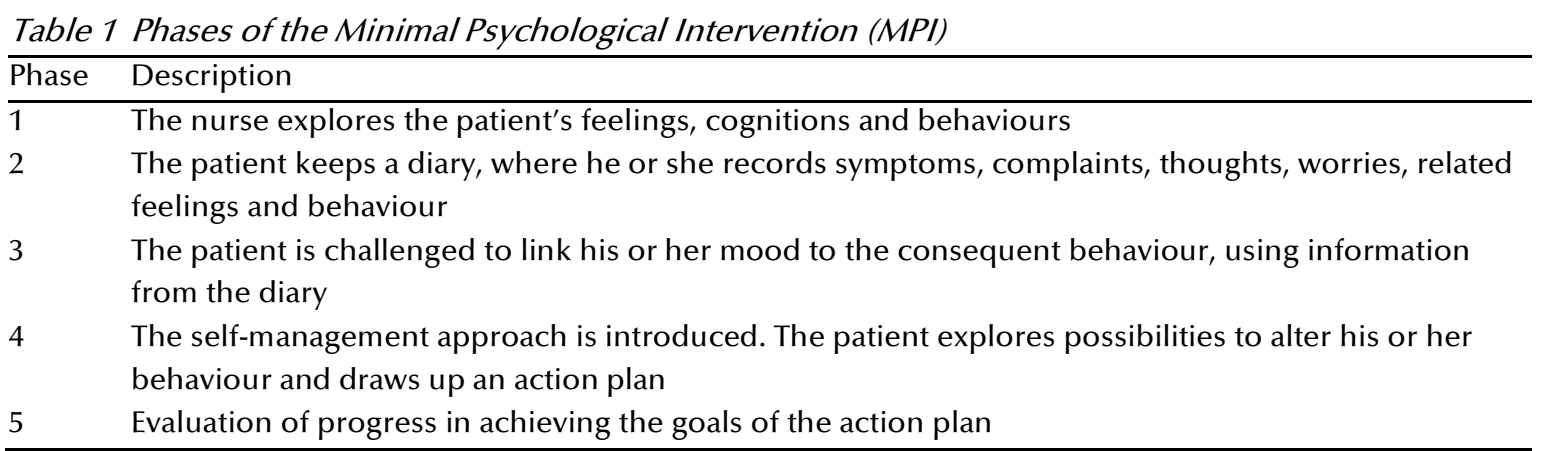

\section{Outcome measures}

Data were collected by means of self-administered questionnaires. Depressive symptoms were assessed using the Beck Depression Inventory (BDI) ${ }^{26}$ and anxiety was assessed using the anxiety subscale of the Symptom Checklist-90 (SCL). ${ }^{27}$ Demographic information was collected at the time of the PHQ-9 screening. Diseasespecific quality of life was assessed using the Saint George's Respiratory Questionnaire (SGRQ).$^{28}$ The SGRQ consists of a total score that summarizes the overall impact of COPD on health status, and three subscales: the activity scale (activities that cause or are limited by breathlessness), the impact scale (social functioning and psychological disturbances) and the symptom scale (effects, frequency and severity of respiratory symptoms). Missing item scores on the SGRQ and SCL were imputed according to the recommendations in their respective manuals; missing items on the BDI were imputed with the patient's mean item score as long as at least $50 \%$ of the items had been completed. 


\section{Analysis}

The power calculation for the DELTA study was based on the BDI in the total group of diabetes and COPD patients. ${ }^{18}$ According to this calculation, assuming an $\alpha$ of 0.05 and a $1-\beta$ (power) of 0.90 , we needed to include $2 \times 96=192$ patients (48 COPD and 48 DM patients in the intervention group and 48 COPD and 48 DM patients in the control group) to allow us to detect a minimum clinically relevant difference of $18 \%$ in improvement ( $\geq 50 \%$ reduction of BDI score relative to baseline).$^{29}$ Anticipating an attrition rate of approximately $30 \%$ and the potential need for subgroup analyses, we decided to recruit a gross number of 360 patients. In the group of COPD patients included in the current study $(n=187)$, a difference of 7.83 on the SGRQ total score would be significant at the $5 \%$ level. A difference of four points on the SGRQ is considered clinically relevant.

Analyses were based on the intention-to-treat principle. The comparability of groups was checked by means of t-tests and Chi-square tests for demographic and outcome variables. Mixed model, repeated-measure ANCOVA analysis was used to test the differences between groups at follow-up measurements. Advantages of mixed model analysis include that it can handle missing observations, and error terms are estimated more precisely, thereby further increasing power. Fixed effects included in the model were: age, gender, educational level, treatment group, baseline value of outcome, time and the product term of time and group. Several random effects and covariance matrices were tested, using -2 log likelihood tests to decide which model had the best fit. Maximum Likelihood models were then run, whose results are presented here.

In addition, effect sizes (d) were calculated by dividing the difference in mean group scores by the pooled standard deviation. ${ }^{30}$ The percentage of patients showing an improvement of four points on the SGRQ total score was also computed and differences between groups were tested with Chi-square tests.

Additional per-protocol analyses were done, including patients who had received a complete intervention (all core steps of the intervention delivered, based on nurses' checklists, $\mathrm{n}=60^{25}$ ) and excluding four control patients who had received information on the content of the intervention and had benefited from this (based on self-report). We also performed analyses in which missing observations were imputed (last observation carried forward - LOCF).

\section{RESULTS}

Figure 1 shows the flow of COPD patients in the trial. Of the 500 patients invited for a MINI, 164 did not meet the inclusion criteria. The 41 patients who were eligible but refused to participate were significantly older than patients who did enter the study. Of the 187 patients who were included, 96 were offered the MPI, and 91 received usual care. The dropout-rate was $36 \%$, which was slightly higher than the anticipated 
EFFECTIVENESS IN COPD

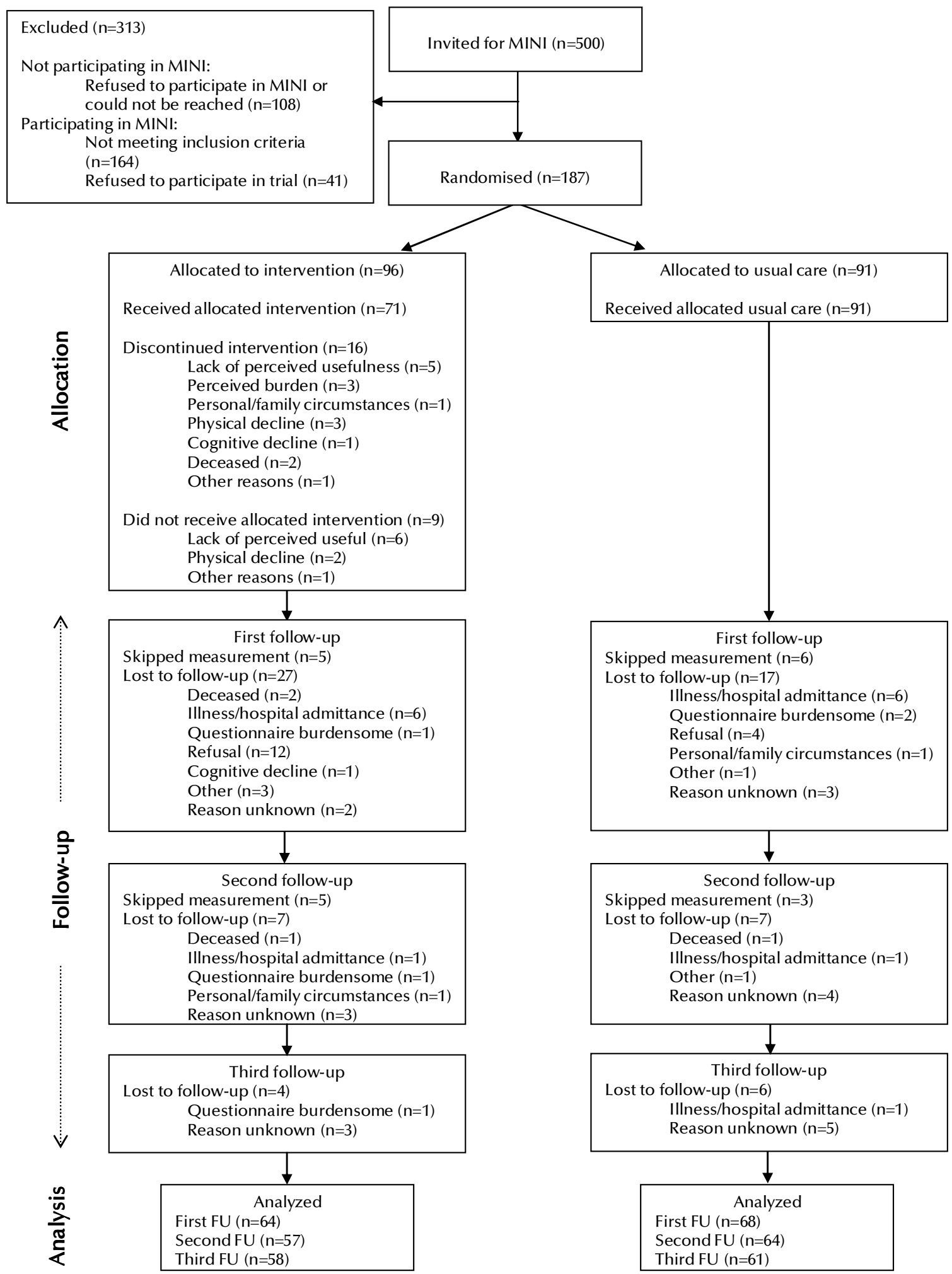

Figure 1 Flowchart 


\section{CHAPTER 6}

$30 \%$. Although the difference was not statistically significant, the dropout-rate was somewhat higher in the intervention group than in the control group $(40 \%$ vs. $33 \%$, $\mathrm{p}=0.35$ ). Furthermore, dropouts overall had a higher age and higher baseline BDI and SGRQ scores (activity and impact subscales and total score), indicating worse depression states and lower quality of life (data not shown).

Table 2 shows the comparability of groups at baseline. None of the differences between the intervention and control groups were statistically significant, although the control group was slightly older and had a somewhat higher educational level than the intervention group. In addition, the control group had somewhat higher mean BDI and SGRQ total scores, indicating a higher level of depressive symptoms and lower quality of life than the intervention group.

Results of the mixed model analyses are presented in Table 3. On average, we found significantly lower scores for both the depression and anxiety outcomes at nine months in the intervention group compared with the control group, indicating fewer symptoms (BDI $p=0.04 ; S C L p=0.003$ ). As for disease-specific quality of life, we found that the intervention group had significantly better scores on the SGRQ activity

Table 2 Comparability of MPI intervention and control groups regarding socio-demographic variables and baseline values of outcomes

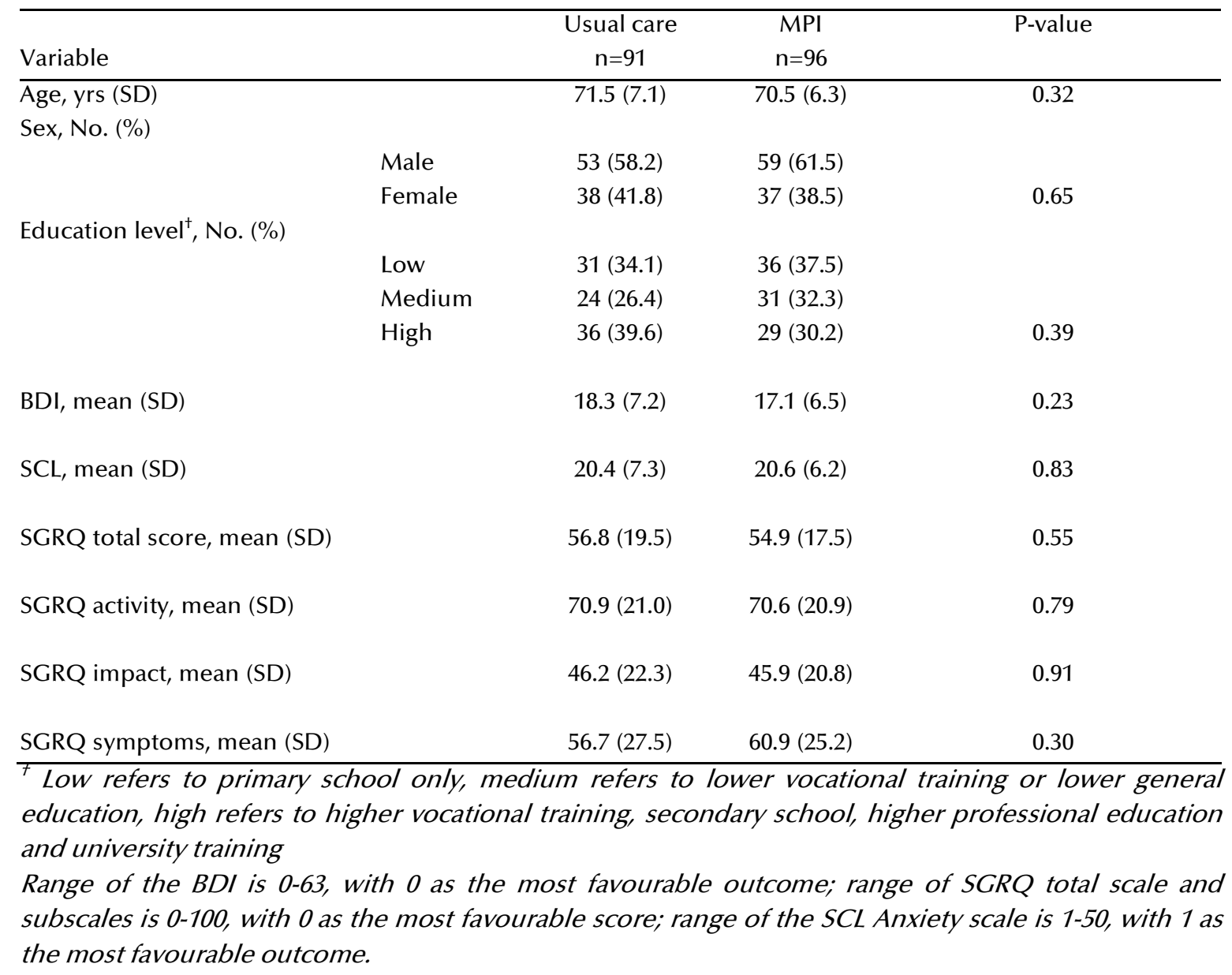


subscale than controls at one week $(p=0.004)$ and three months $(p=0.02)$ after the intervention, but the mean difference between groups was no longer significant at nine months after the intervention $(p=0.09)$. Results for the SGRQ impact subscaleshowed the opposite pattern: there were no significant differences immediately after the intervention, but the intervention group had significantly better scores than the control group at three months $(p=0.02)$ and nine months $(p=0.003)$ after the intervention.

Table 3 Outcomes on quality of life, depression and anxiety

\begin{tabular}{|c|c|c|c|c|c|c|c|}
\hline & $\begin{array}{l}\text { Usual Care } \\
\qquad \mathrm{N}=91\end{array}$ & & $\begin{array}{c}\mathrm{MPI} \\
\mathrm{N}=96\end{array}$ & & $\begin{array}{c}\text { Mean difference } \\
\text { (UC-MPI) }\end{array}$ & & $\begin{array}{l}\text { Effect } \\
\text { size }\end{array}$ \\
\hline & Mean $(\mathrm{SE})^{*}$ & $\mathrm{~N}^{* *}$ & Mean $(\mathrm{SE})^{*}$ & $\mathrm{~N}^{* *}$ & $(95 \% \mathrm{Cl})$ & P-value & $\mathrm{d}$ \\
\hline \multicolumn{8}{|c|}{ Depression and anxiety scales } \\
\hline \multicolumn{8}{|c|}{ BDI } \\
\hline After 1 week & $17.31(0.77)$ & 68 & $15.45(0.80)$ & 64 & $1.85(-0.36$ to 4.06$)$ & 0.10 & 0.29 \\
\hline After 3 months & $17.57(0.82)$ & 63 & $15.59(0.87)$ & 57 & $1.98(-0.39$ to 4.36$)$ & 0.10 & 0.31 \\
\hline After 9 months & $17.96(0.96)$ & 59 & $15.04(1.00)$ & 58 & $2.92(0.17$ to 5.68$)$ & 0.04 & 0.39 \\
\hline \multicolumn{8}{|l|}{ SCL Anxiety } \\
\hline After 1 week & $21.43(0.69)$ & 66 & $20.76(0.73)$ & 61 & $0.67(-1.31$ to 2.64$)$ & 0.51 & 0.11 \\
\hline After 3 months & $21.66(0.74)$ & 61 & $20.87(0.78)$ & 55 & $0.79(-1.34$ to 2.91$)$ & 0.47 & 0.14 \\
\hline After 9 months & $23.54(0.84)$ & 58 & $19.85(0.87)$ & 57 & 3.69 (1.29 to 6.09$)$ & 0.003 & 0.57 \\
\hline \multicolumn{8}{|l|}{ SGRQ subscales } \\
\hline \multicolumn{8}{|l|}{ Activity subscale } \\
\hline After 1 week & $70.51(1.75)$ & 54 & $62.99(1.92)$ & 46 & 7.52 (2.39 to 12.66$)$ & 0.004 & 0.59 \\
\hline After 3 months & $68.91(1.86)$ & 48 & $62.54(2.07)$ & 41 & 6.37 (0.87 to 11.87$)$ & 0.02 & 0.49 \\
\hline After 9 months & $70.64(2.09)$ & 47 & $65.32(2.25)$ & 45 & $5.32(-0.78$ to 11.43$)$ & 0.09 & 0.37 \\
\hline \multicolumn{8}{|l|}{ Impact subscale } \\
\hline After 1 week & 44.95 (1.58) & 60 & $41.43(1.70)$ & 52 & $3.52(-1.03$ to 8.07$)$ & 0.13 & 0.29 \\
\hline After 3 months & $46.51(1.83)$ & 51 & $40.16(1.93)$ & 49 & 6.35 (1.10 to 11.61$)$ & 0.02 & 0.48 \\
\hline After 9 months & $46.48(1.96)$ & 50 & $37.86(2.03)$ & 49 & 8.62 (3.04 to 14.21$)$ & 0.003 & 0.62 \\
\hline \multicolumn{8}{|l|}{ Symptoms subscale } \\
\hline After 1 week & $60.08(2.53)$ & 64 & $57.58(2.67)$ & 57 & $2.50(-4.71$ to 9.70$)$ & 0.50 & 0.12 \\
\hline After 3 months & $59.16(2.61)$ & 58 & $53.91(2.84)$ & 48 & 5.25 (-2.33 to 12.84$)$ & 0.17 & 0.27 \\
\hline After 9 months & $57.48(2.67)$ & 54 & $52.37(2.75)$ & 52 & 5.11 (-2.43 to 12.65$)$ & 0.18 & 0.26 \\
\hline \multicolumn{8}{|l|}{ Total SGRQ scale } \\
\hline After 1 week & $55.63(1.57)$ & 49 & $50.48(1.71)$ & 42 & $5.14(0.59$ to 9.70$)$ & 0.03 & 0.47 \\
\hline After 3 months & 56.09 (1.64) & 44 & $49.12(1.82)$ & 38 & 6.97 (2.14 to 11.80$)$ & 0.005 & 0.64 \\
\hline After 9 months & $56.08(1.86)$ & 42 & $48.14(1.98)$ & 42 & 7.94 (2.67 to 13.32$)$ & 0.004 & 0.65 \\
\hline
\end{tabular}

Abbreviations: SE, standard error; BDI, Beck Depression Inventory; SCL Anxiety, Symptom Checklist Anxiety; SGRQ, Saint George's Respiratory Questionnaire

Range of the BDI is 0-63, with 0 as the most favourable outcome; range of SGRQ total scale and subscales is 0-100, with 0 as the most favourable score; range of the SCL Anxiety scale is 1-50, with 1 as the most favourable outcome.

*estimates from mixed model regression analyses, corrected for age, gender, level of education and baseline value of the outcome measure.

**The maximum number of patients across measurements for whom imputation of items was not possible was 2 for the BDI, 5 for the SCL, 32 for Activity, 21 for Impact, 15 for Symptoms and 41 for the SGRQ total score. 
No effects were found on the SGRQ symptom subscale, but the SGRQ total scores showed that, on average, intervention patients had a significantly better quality of life than controls at all follow-up measurements $(1 \mathrm{wk} p=0.03 ; 3$ months $p=0.005 ; 9$ months $\mathrm{p}=0.004)$. In terms of clinical relevance, a four-point change relative to baseline on the SGRQ total score was more often seen in intervention patients than in control patients at all follow-up measurements, with a significant difference at nine months ( $52 \%$ vs. $29 \%$, $p=0.03$, not tabulated). Effect sizes corresponding to significant results can all be classified as medium. ${ }^{30}$ Additional per-protocol and LOCF analyses produced similar results (data not shown).

\section{DISCUSSION}

This study showed that a nurse-led minimal psychological intervention significantly reduced depressive symptoms and prevented aggravation of anxiety in elderly COPD patients with minor to moderate depression. The intervention also improved diseasespecific quality of life. Corresponding effect sizes were all medium. ${ }^{30}$

Our tailor-made intervention was intended to educate patients to take responsibility for the day-to-day management of their own illness and its consequences. This was achieved by improving patients' understanding of the relation between their behaviour and consequent mood, by challenging them to draw up action plans to change their behaviour, and by teaching them self-management skills that they could apply in daily life. The study results indicate that the aim of the intervention was indeed achieved, as differences between groups became larger over time for all outcomes, except for the Activity subscale. This may very well mean that patients learned skills and continued to apply them in daily life during the follow-up period, even becoming more successful over time. Winning back control over daily symptoms by learning coping skills seems very rewarding especially for COPD patients, as many of them have developed a tendency towards 'learned helplessness' during the course of their disease.

As noted by Coventry \& Gellatly in their recent review, ${ }^{16}$ only a few studies have evaluated the effectiveness of psychological treatments for depression and anxiety in COPD patients. The effect sizes for symptoms of depression and anxiety found in our study are highly similar to those reported by Coventry and Gellatly. ${ }^{16}$ As regards disease-specific quality of life, the group difference of 7.94 for the SGRQ total score in our study is higher than the weighted mean difference (WMD) derived from seven other studies evaluating self-management $(\mathrm{WMD}=2.58 ; 95 \% \mathrm{Cl}-0.02-5.14),{ }^{17}$ and is also larger than the accepted cut-off point (four points) for a clinically relevant change. ${ }^{31}$ Interestingly, whereas previous analyses found no effect on generic quality of life, ${ }^{18}$ we did find an effect on disease-specific quality of life in the current analyses. Disease-specific quality of life measures have been shown to be more responsive to change than generic measures. ${ }^{32}$ Since the SGRQ covers specific domains that are important to patients with COPD, the use of this measure enabled us to detect 
changes in quality of life that are of specific importance and relevance to them but are beyond the scope of generic instruments.

Several potential weaknesses of our study should be considered. First, dropouts were older and had a lower quality of life and higher levels of depressive symptoms. Although dropout was somewhat more common in the intervention group, this difference was not statistically significant. Despite the use of mixed models for repeated measures (which include patients with incomplete observations), this pattern of attrition might have affected our findings. However, an LOCF analysis, often regarded as a conservative imputation method, ${ }^{33}$ did not alter our conclusions. Second, disease severity can be an interfering factor if severity is unequally divided between groups. We did not have objective data on the severity of COPD among participants at baseline, such as FEV1 data, since patient inclusion was done by GPs on the basis of existing practice records. However, disease-specific quality of life, which has been shown to be related to COPD severity but may reflect disease impact better, ${ }^{34}$ did not differ between groups at baseline. Third, blinding of patients was obviously not possible, as we compared the intervention with usual care, as is often done in pragmatic trials.

Our study had several strengths. Suspected depression, as picked up by the screening questionnaire, was confirmed by a DSM-IV based diagnostic interview. Additionally, we included patients with a wide range of depression severity, so that our sample reflects the natural heterogeneity of patients with depressive symptoms in primary care. A further strength of our approach is that nurses were used to administer the intervention. Since nurses are already involved in disease-management programmes and as such see patients on a regular basis, they seem obvious candidates for the task of administering psychological interventions. We have shown that nurses without any specific psychiatric background can be trained to administer an MPI that successfully reduces depression and anxiety and improves quality of life. An extensive process evaluation showed that nurses had adhered closely to the protocol and that they were highly enthusiastic about the intervention. As for patients, the evaluation revealed that they were also highly satisfied with the intervention and would recommend it to other persons with a chronic illness. ${ }^{25}$ Incorporation of our intervention within existing disease-management programmes therefore appears a logical next step. By taking on the task of depression management within these programmes, nurses may thus complement the GP's regular work.

\section{CONCLUSION}

In a time when aging of the population is likely to increase the prevalence and burden of COPD, maintaining the highest possible quality of life and slowing down further deterioration of the COPD patient's health status will remain the key goals of COPD treatment. Our nurse-administered minimal psychological intervention was effective in reducing anxiety and depressive symptoms and improving disease- 


\section{CHAPTER 6}

specific quality of life. We therefore feel that adding MPI to existing diseasemanagement programmes is likely to improve the care for elderly COPD patients.

\section{Acknowledgement}

We want to thank our nurses Kitty Daemen, Henny Geelen, Francine Hendriks and Mieke Witte for administering the intervention with great dedication and enthusiasm, as well as Wendy Engering for her contribution to patient recruitment and Dorien Mintjes for her contribution to data entry, and MEMIC (Centre for Data and Information Management) for providing us with a data management system. his study was funded by the Netherlands Organisation for Health Research and Development (ZonMw), programme on Health Care Efficiency Research, grant number 945-03-047. 


\section{REFERENCES}

1. Halbert RJ, Natoli JL, Gano A, Badamgarav E, Buist AS, Mannino DM. Global burden of COPD: systematic review and meta-analysis. Eur Respir J2006;28(3):523-32.

2. Kunik ME, Roundy K, Veazey C, Souchek J, Richardson P, Wray NP, et al. Surprisingly high prevalence of anxiety and depression in chronic breathing disorders. Chest 2005;127(4):1205-11.

3. Mikkelsen RL, Middelboe T, Pisinger C, Stage KB. Anxiety and depression in patients with chronic obstructive pulmonary disease (COPD). A review. Nord J Psychiatry 2004;58(1):65-70.

4. Gudmundsson G, Gislason T, Janson C, Lindberg E, Suppli Ulrik C, Brondum E, et al. Depression, anxiety and health status after hospitalisation for COPD: a multicentre study in the Nordic countries. Respir Med 2006;100(1):87-93.

5. Hynninen KM, Breitve MH, Wiborg AB, Pallesen S, Nordhus IH. Psychological characteristics of patients with chronic obstructive pulmonary disease: a review. J Psychosom Res 2005;59(6):429-43.

6. Yohannes AM, Baldwin RC, Connolly MJ. Prevalence of sub-threshold depression in elderly patients with chronic obstructive pulmonary disease. Int J Geriatr Psychiatry 2003;18(5):412-6.

7. Gan WQ, Man SF, Senthilselvan A, Sin DD. Association between chronic obstructive pulmonary disease and systemic inflammation: a systematic review and a meta-analysis. Thorax 2004;59(7):574-80.

8. Maes M. Evidence for an immune response in major depression: a review and hypothesis. Prog Neuropsychopharmacol Biol Psychiatry 1995;19(1):11-38.

9. Herbert TB, Cohen S. Depression and immunity: a meta-analytic review. Psycho/ Bul/1993;113(3):472-86.

10. Cully JA, Graham DP, Stanley MA, Ferguson CJ, Sharafkhaneh A, Souchek J, et al. Quality of life in patients with chronic obstructive pulmonary disease and comorbid anxiety or depression. Psychosomatics 2006;47(4):312-9.

11. Norwood R, Balkissoon R. Current perspectives on management of co-morbid depression in COPD. Copd 2005;2(1):185-93.

12. Brenes GA. Anxiety and chronic obstructive pulmonary disease: prevalence, impact, and treatment. Psychosom Med 2003;65(6):963-70.

13. Yohannes AM, Baldwin RC, Connolly MJ. Depression and anxiety in elderly patients with chronic obstructive pulmonary disease. Age Ageing 2006;35(5):457-9.

14. Hunkeler EM, Meresman JF, Hargreaves WA, Fireman B, Berman WH, Kirsch AJ, et al. Efficacy of nurse telehealth care and peer support in augmenting treatment of depression in primary care. Arch Fam Med 2000;9(8):700-8.

15. Mynors-Wallis LM, Gath DH, Day A, Baker F. Randomised controlled trial of problem solving treatment, antidepressant medication, and combined treatment for major depression in primary care. BMJ 2000;320(7226):26-30.

16. Coventry PA, Gellatly JL. Improving outcomes for COPD patients with mild-to-moderate anxiety and depression: A systematic review of cognitive behavioural therapy. Br / Health Psycho/ Published Online First: 18 april 2007; doi:10.1348/135910707X203723.

17. Effing T, Monninkhof EM, van der Valk PD, van der Palen J, van Herwaarden CL, Partidge MR, et al. Selfmanagement education for patients with chronic obstructive pulmonary disease. Cochrane Database Syst Rev 2007(4):CD002990.

18. Lamers F, Jonkers CCM, Bosma H, Kempen GIJM, Meijer JAMJ, Penninx BWJH, et al. The effectiveness of a minimal psychological intervention in chronically ill elderly patients with depression: a randomised trial (the DELTA-study). submitted.

19. Lamers F, Jonkers CC, Bosma H, Diederiks JP, van Eijk JT. Effectiveness and cost-effectiveness of a minimal psychological intervention to reduce non-severe depression in chronically ill elderly patients: the design of a randomised controlled trial [ISRCTN92331982]. BMC Public Health 2006;6(1):161.

20. Spitzer RL, Kroenke K, Williams JB. Validation and utility of a self-report version of PRIME-MD: the PHQ primary care study. Primary Care Evaluation of Mental Disorders. Patient Health Questionnaire. JAMA 1999;282(18):1737-44.

21. Sheehan DV, Lecrubier $\mathrm{Y}$, Sheehan $\mathrm{KH}$, Janavs J, Weiller E, Keskiner A, et al. The validity of the Mini International Neuropsychiatric Interview (MINI) according to the SCID-P and its reliability. Eur Psychiatry 1997;12(5):232-241. 


\section{CHAPTER 6}

22. Hamilton M. A rating scale for depression. J Neurol Neurosurg Psychiatry 1960;23:56-62.

23. Geijer RMM, Thiadens HA, Smeele IJM, Sachs APE, Bottema BJAM, van Hensbergen W, et al. NHGStandaard COPD en Astma bij Volwassenen: Diagnostiek [NHG Practice Guideline COPD: Diagnosis]. Huisarts Wet 2001;44(3):107-117.

24. Van Eijk JT, Diederiks JP, Kempen GI, Honig A, van der Meer K, Brenninkmeijer WJ. Development and feasibility of a nurse administered strategy on depression in community-dwelling patients with a chronic physical disease. Patient Educ Couns 2004;54(1):87-94.

25. Jonkers CCM, Lamers F, Bosma H, Metsemakers JF, Kempen GIJM, van Eijk JTM. Process evaluation of a minimal psychological intervention to reduce depression in chronically ill elderly persons. Patient Educ Couns 2007;68(3):252-257.

26. Beck AT, Ward CH, Mendelson M, Mock J, Erbaugh J. An inventory for measuring depression. Arch Gen Psychiatry1961;4:561-571.

27. Arrindel WA, Ettema JHM. SCL-90: Handleiding bij een multidimensionele psychopathalogie-indicator. Lisse: Swets en Zeitlinger B.V., 1986.

28. Jones PW, Quirk FH, Baveystock CM, Littlejohns P. A self-complete measure of health status for chronic airflow limitation. The St. George's Respiratory Questionnaire. Am Rev Respir Dis 1992;145(6):1321-7.

29. Pocock S. Clinical trials: A Practical Approach. Chichester: John Wiley and Sons, 1983.

30. Cohen J. Statistical power analysis for the behavioral sciences. 2nd edition ed. Hillsdale, NJ: Lawrence Erlbaum Associates, 1988.

31. Jones PW. St. George's Respiratory Questionnaire: MCID. Copd 2005;2(1):75-9.

32. Puhan MA, Guyatt GH, Goldstein R, Mador J, McKim D, Stahl E, et al. Relative responsiveness of the Chronic Respiratory Questionnaire, St. Georges Respiratory Questionnaire and four other health-related quality of life instruments for patients with chronic lung disease. Respir Med 2007;101(2):308-16.

33. Beunckens C, Molenberghs G, Kenward MG. Direct likelihood analysis versus simple forms of imputation for missing data in randomized clinical trials. Clin Trials 2005;2(5):379-86.

34. Stahl E, Lindberg A, Jansson SA, Ronmark E, Svensson K, Andersson F, et al. Health-related quality of life is related to COPD disease severity. Health Qual Life Outcomes 2005;3:56. 


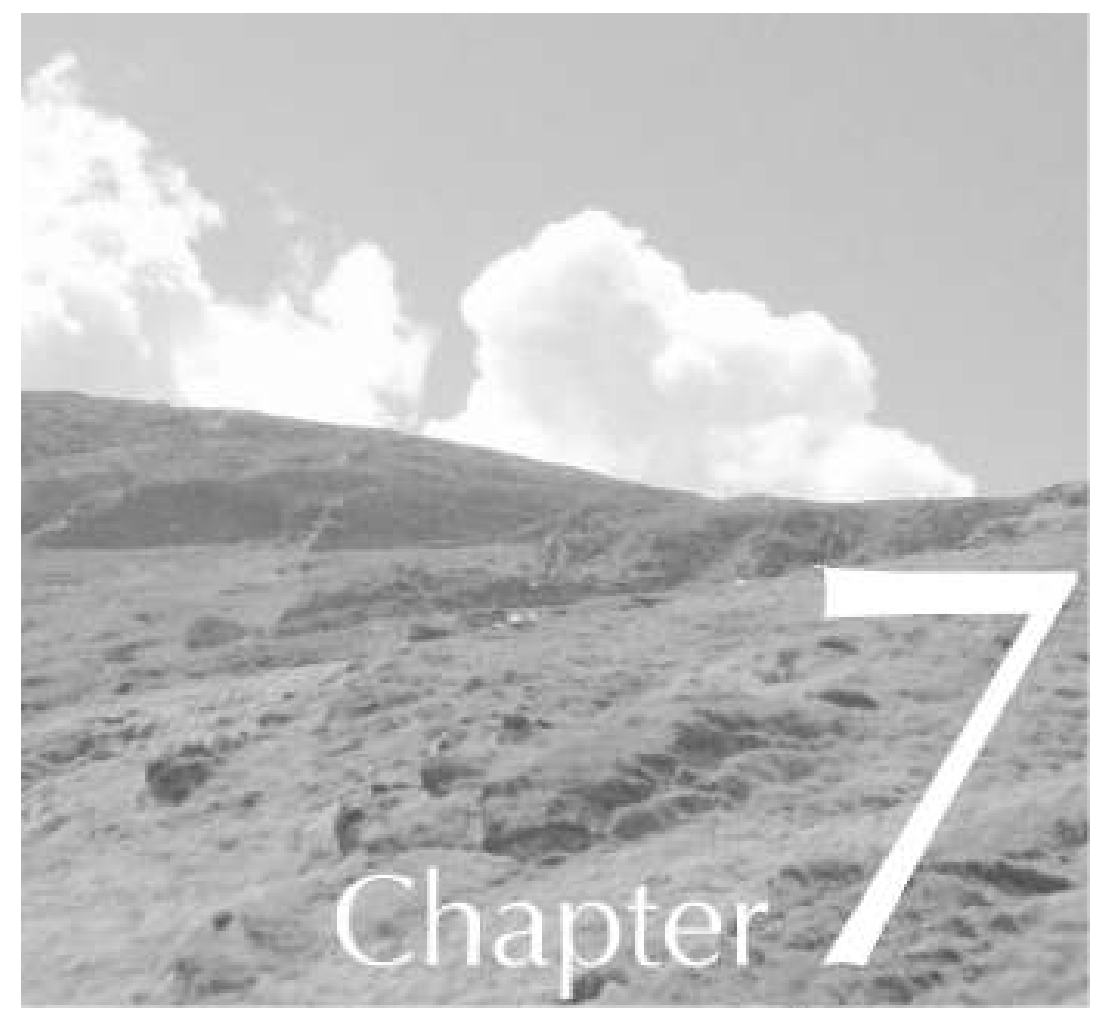

Economic evaluation of a minimal psychological intervention in chronically ill elderly patients with depression: a randomised trial (the DELTA-study) 
CHAPTER 7 


\section{ABSTRACT}

Objective: Depression is associated with high healthcare utilization and related costs. Effective treatments might reduce the economic burden. The objective of this study was to establish the cost-utility of a minimal psychological intervention (MPI) aimed at reducing depression and improving quality of life in elderly persons with diabetes or chronic obstructive pulmonary disease and co-occurring minor, mild or moderate depression.

Method: Trial-based cost-utility analysis comparing the MPI with usual care. Annual costs and quality-adjusted life years (QALYs) based on the Euroqol (EQ5D) and on depression-free days were calculated.

Results: We found improvements, albeit not significant, in clinical outcomes and a decrease in mean annual costs in favour of the MPI group. Additional bootstrap analysis indicated a dominant intervention, with a probability of $63 \%$ that the MPI is less costly and more effective than usual care.

Conclusion: This study tends to support further dissemination of the MPI in regular care. 
CHAPTER 7 
Depression is a common disorder in older persons ${ }^{1}$ and is associated with a reduced quality of life, ${ }^{2}$ increased morbidity, ${ }^{3}$ and increased physical disability. ${ }^{4}$ This applies especially to older persons with chronic illnesses, such as type II diabetes mellitus (DM) and chronic obstructive pulmonary disease (COPD). ${ }^{56}$ Patients with depression run the risk of sliding into a downward spiral, since depression and disability are mutually reinforcing. ${ }^{4}$ Depression may impair one's ability to adhere to medical regimens (diet, exercise, quitting smoking, taking medication regularly), potentially worsening the course of the chronic illness, ${ }^{7}$ and may also lead to greater health care utilization and related costs. ${ }^{8} 9$ It is therefore of great importance to develop treatments for chronically ill elderly persons that help reduce the burden of depression. This is especially true for the treatment of depression in primary care, since persons with depression often present initially to a general practitioner. ${ }^{8}$

Although attempts have been made to study the cost-effectiveness of collaborative care depression treatment, offering both pharmacological and psychological treatment options, ${ }^{8-11}$ the societal impact of psychological depression treatment among elderly persons with chronic somatic illnesses, incorporating health care costs as well as production losses, remains largely unclear. $^{8}$ The Depression in Elderly with LongTerm Afflictions (DELTA) study has been designed to evaluate the effectiveness and cost-effectiveness of a minimal psychological intervention (MPI) to reduce depression and improve quality of life in elderly persons with DM or COPD and co-occurring depression. The MPI was carried out by primary care nurses and is based on principles of self-management and cognitive behavioural therapy (CBT).

The aim of this article is to assess, from a societal perspective, the cost-effectiveness of the MPI compared to usual care. We expected our intervention to lead to an equal level of health care utilization as that with usual care, and therefore to equal healthrelated costs. In addition, we expected favorable outcomes in terms of quality of life and depression, implying a cost-effective intervention.

\section{METHODS}

\section{Design}

The economic evaluation was embedded in a two-armed randomised controlled trial (RCT). A detailed description of the design of the DELTA-study has been published elsewhere. ${ }^{12}$ A block randomisation was used, with stratification for chronic illness and the general practice where the patient was registered. We used a block size of two, because we expected to include few patients per general practice and wanted equal distribution over the groups. The researchers entered each patient's identification number into a computer system connected to an external agency. Patients were then randomised by the agency, using a computerized random number generator. Data were entered by the researchers, who were blinded for the allocation. Costs and effects were assessed at baseline (prior to randomisation) and at three, six, nine (only 
costs), and twelve months after baseline. The first follow-up in the intervention group was one week after the final intervention contact, and depended upon the duration of the tailor-made intervention. The economic evaluation was performed from a societal perspective, which implies that all relevant costs and effects are taken into account. ${ }^{13}$ Approval for this study was granted by the Medical Ethics Committee of Maastricht University/University Hospital Maastricht.

\section{Participants}

Between October 2003 and May 2005, participants were recruited in 89 primary care practices in the south of the Netherlands. Patients with an established diagnosis of DM or COPD, aged 60 years and over, who were community-dwelling and did not meet exclusion criteria (treatment with antidepressants for depression, major psychiatric problems, current psychosocial/psychiatric treatment, serious cognitive problems, being on a waiting list for nursing home, being bedridden, loss of spouse within the last three months, and not being fluent in Dutch) were sent the Patient Health Questionnaire (PHQ-9). ${ }^{14}$ Patients who reported having at least two symptoms of depression present for more than half of the days, one of them being loss of interest or depressed mood, were invited to take part in a structured diagnostic interview for DSM-IV axis I disorders, the Mini International Neuropsychiatric Interview (MINI). ${ }^{15}$ In addition, the Hamilton Depression Rating Scale (HDRS) ${ }^{16}$ was used to determine the severity of the depression. The MINI and HDRS were administered at the patients' home by trained nurses. Patients with minor depression, mild to moderate major depression or dysthymia were invited to participate in the trial. Patients with suicidal risk and patients with severe major depression (HDRS>18) were excluded and referred to their GP. After signing an informed consent form and completing a baseline questionnaire, patients were randomly allocated to the MPI $(\mathrm{N}=183)$ or usual care $(\mathrm{N}=178)$.

\section{Intervention and usual care}

Patients allocated to the intervention group received the MPI supplementary to usual care. The intervention was delivered at the patient's home by nurses, who were trained in the DELTA intervention, based on principles of CBT and self-management, but had not received additional training for DM or COPD. The DELTA intervention consists of five phases, which have been described in more detail elsewhere. ${ }^{12} 17$ Briefly, in phase one, the nurse explores the patient's feelings, cognitions, and behaviours. During phase two, the patient keeps a diary, in which they record symptoms, complaints, thoughts, worries, and related feelings and behaviours. In phase three, the patient is challenged to link their mood to the consequent behaviour, using information from the diary. The self-management approach is introduced in phase four, where the patient explores possibilities to alter their behaviour and where they draw up an action plan. Phase five consists of an evaluation of the degree 
to which goals from the action plan have been achieved. The intervention is tailormade and a home visit could comprise one or more phases. During the study, patients received two to ten visits over a period of at most three months, depending on the patient's progress. The mean number of visits was four, with a mean duration of 61 minutes. ${ }^{18}$

Patients assigned to usual care received regular treatment according to the practice guidelines of their chronic somatic illness. These practice guidelines, produced by the Dutch College of General Practitioners, encompass regular check-ups for medical symptoms, but do not involve detection and treatment of depressive symptoms. ${ }^{19-21}$ Care providers remained blinded for the results of the depression screening for the duration of the study. Due to blinding of the care providers and the randomisation, co-interventions are supposed to be comparable between intervention and control group.

\section{Measurements}

\section{COSTS}

To establish the costs, relevant cost items were identified, after which these costs were measured and values were placed on the cost items.

We started by identifying program costs, health care costs, patient and family costs, and productivity losses. Program costs include the costs that can be attributed to the process of developing and administering the MPI, for example the costs of the home visits, MPI training for nurses, and nurses' travel expenses. Research-specific costs, such as costs of questionnaires, were excluded. Health care costs in our study were all costs related to patients' visits to a GP's surgery, hospital care (inpatient and outpatient), allied health professionals such as physiotherapists or dieticians, professional home care, medical devices and assistive devices, and prescribed and over-thecounter medication. Patient and family costs included costs of informal care (help from family and friends) and paid domestic help. Productivity losses consisted of sick leave from work and loss of activities in and around the home.

We then measured the cost categories identified above. The program costs were measured by means of a questionnaire in which nurses recorded time spent on home visits and travelling. The time spent developing the MPI and training nurses to use it was recorded by the researchers. Payroll information was used to calculate the hourly wages of nurses, developers, and trainers. Cost diaries ${ }^{22}$ were used to measure volumes of health care utilization, patient and family costs, and productivity losses. Patients kept a prospective diary for two weeks at baseline and for four weeks at all four follow-up measurements. After each measurement, a telephone operator, blinded for allocation, contacted patients to retrieve information from the diary. Data were immediately entered in a computer file to ensure efficiency and reliability.

Finally, the valuation was based on volumes obtained from the cost diary and questionnaires, multiplied by cost prices derived from the updated Dutch manual for costing. ${ }^{23}{ }^{24}$ Costs due to productivity losses were estimated using the friction cost 
approach, as described in this manual. Prices of informal care were based on shadow prices for unpaid work. Where no standard cost prizes were available, real costs or tariffs were used to estimate costs. For example, costs of assistive devices were obtained from the market prices of these devices (volumes and cost price details are available upon request). Baseline costs were used to examine the comparability of the groups at baseline. The total annual costs were determined by extrapolating the costs from the available four months of follow-up measurements by three to obtain the total costs during twelve months of follow-up. The annual costs are presented in Euros and the baseline year was 2004. The discounting rate was $4 \%{ }^{23} 24$

\section{EFFECTS}

The generic effects on quality of life were assessed with the Euroqol (EQ5D). ${ }^{25}$ This widely used quality-of-life instrument includes five dimensions of health-related quality of life, namely mobility, self-care, daily activities, pain/discomfort and depression/anxiety. Each dimension was rated at three levels: no problems, some problems and major problems. The five dimensions were combined into a health state. Utility values were calculated for these health states, using preferences elicited from a general Dutch population. ${ }^{26} 27$ The utility values were used to compute qualityadjusted life years (QALY-EQ5D) by means of the area under the curve method. ${ }^{28}$ In addition, depression-specific effects were assessed with the Beck Depression Inventory (BDI). ${ }^{29}$ We used the method developed by Lave et al. to calculate depression-free days (DFD) during follow-up. ${ }^{30}$ This method uses depression scores from the BDI over time to estimate days free of significant depressive symptoms. As suggested in previous research, we used a health utility improvement of 0.4 for depression-free days to estimate the QALY-DFD. ${ }^{3031}$

The EQ5D and BDI were assessed by means of self-administered questionnaires, sent together with the cost diaries, at baseline and at the three, six, and twelve months' follow-up assessments.

\section{Analyses}

Analyses were based on the intention-to-treat principle. An analysis of baseline characteristics and baseline costs examined the comparability of the groups at baseline. Persons with effect scores on at least two of the three follow-up measurements and cost data on at least three of the four follow-up measurements were included in the analysis. Missing items on the BDI scale were replaced by the individual's own mean of non-missing items at that follow-up measurement, if at least half of the items for that follow-up were available. This method could not be applied to the EQ5D and cost data, since domains of the EQ5D and cost category levels (e.g. hospital-related costs or costs of informal care) consisted of one item. Therefore, missing data on the EQ5D and cost category levels were replaced by the individual's own mean of non-missing data at follow-up measurements. Using person specific mean 
imputation techniques is considered a valid method for imputation of longitudinal data. $^{32}$

Because cost data are typically highly skewed, we used bootstrap estimation with 1000 replications to obtain means and standard deviations of our cost and effect data. The differences between the intervention and control groups were estimated by means of linear regression, controlling for age, sex, education, chronic somatic illness, and baseline value of either the cost category or the effect measure.

\section{Cost-utility analysis}

To establish the cost-utility of our intervention, we bootstrapped the predicted annual total cost and the predicted effects (QALY-EQ5D and QALY-DFD) derived from the linear regression models. The incremental cost-utility ratio (ICUR) was calculated as:

$\operatorname{ICUR}=(\mathrm{Ci}-\mathrm{CC}) /(\mathrm{Ei}-\mathrm{Ec})$,

where $\mathrm{Ci}$ is the adjusted annual total cost of the intervention group, $\mathrm{Cc}$ is the adjusted annual total cost of the control group, $\mathrm{Ei}$ is the adjusted effect for the intervention group and Ec is the adjusted effect for the control group.

In addition to the primary (QALY-EQ5D) and secondary (QALY-DFD) analyses, we evaluated two models to examine the sensitivity of our cost-utility results. First, we conducted an analysis in which missing cost data were not imputed (complete case analysis). A second sensitivity analysis was performed to test whether a reduction of program cost from $€ 337$ to $€ 282$ would change the cost-utility ratio. The reduction of program costs was based on a scenario in which patients visit the nurse at the GP's surgery instead of the nurses paying home visits, as was done in our study. This scenario is considered to be a suitable alternative when implementing our intervention in routine practice.

\section{RESULTS}

Of the 361 eligible patients, 183 were assigned to the intervention group and 178 to the control group. After imputation, complete data were available for 228 persons (control $n=118$; intervention $n=110)$. These persons were significantly younger $(p<.00)$ and had significantly higher utility scores at baseline $(p=.01)$ than persons for whom complete follow-up data was not available. No other significant differences in characteristics were found between these groups.

Table 1 shows the baseline characteristics of the intervention and control groups. The intervention group had slightly higher costs than the control group in the two weeks prior to the intervention, but differences were not significant. Other characteristics were comparable between groups. 


\section{CHAPTER 7}

Table 1 Comparability of intervention and control groups in terms of socio-demographic variables and baseline values of outcomes

\begin{tabular}{|c|c|c|c|c|}
\hline Variable & & $\begin{array}{c}\text { Usual Care } \\
\mathrm{n}=118\end{array}$ & $\begin{array}{c}\mathrm{MPI} \\
\mathrm{n}=110\end{array}$ & P-value \\
\hline Age, yrs (SD) & & $69.98(6.26)$ & $69.47(6.17)$ & .54 \\
\hline \multirow[t]{3}{*}{ Sex, No. $(\%)$} & & & & .97 \\
\hline & Male & $63(53.4)$ & $59(53.6)$ & \\
\hline & Female & $55(46.6)$ & $51(46.4)$ & \\
\hline \multirow[t]{3}{*}{ Chronic illness, No. (\%) } & & & & .72 \\
\hline & Diabetes & $64(54.2)$ & $57(51.8)$ & \\
\hline & COPD & $54(45.8)$ & $53(48.2)$ & \\
\hline \multirow[t]{4}{*}{ Education level*, No. (\%) } & & & & .25 \\
\hline & Low & $41(34.7)$ & $34(30.9)$ & \\
\hline & Medium & $26(22.0)$ & $35(31.8)$ & \\
\hline & High & $51(43.2)$ & $41(37.3)$ & \\
\hline \multicolumn{2}{|l|}{ Utility $^{\dagger}$, mean $(\mathrm{SD})$} & $0.63(0.20)$ & $0.61(0.22)$ & .35 \\
\hline \multicolumn{2}{|l|}{$\mathrm{BDI}^{\ddagger}$, mean $(\mathrm{SD})$} & $17.48(8.07)$ & $16.73(7.20)$ & .46 \\
\hline \multicolumn{2}{|l|}{$\begin{array}{l}\text { Costs prior } 2 \mathrm{wk} \\
\text { mean Euro (SD) }\end{array}$} & $307(30)$ & $337(37)$ & .56 \\
\hline \multicolumn{5}{|c|}{$\begin{array}{l}\text { * Low refers to primary school only, medium refers to lower vocational training or lower general } \\
\text { education, high refers to higher vocational training, general secondary education, higher professional } \\
\text { education and university training } \\
{ }^{+} \text {Based on the Dutch algorithm for the EQ5D scores; utility scores range from } 0 \text { (death) to } 1 \text { (full health) } \\
{ }^{*} \text { Range of the BDI is 0-63, with } 0 \text { as the most favorable outcome }\end{array}$} \\
\hline
\end{tabular}

Table 2 Mean annual cost per patient*

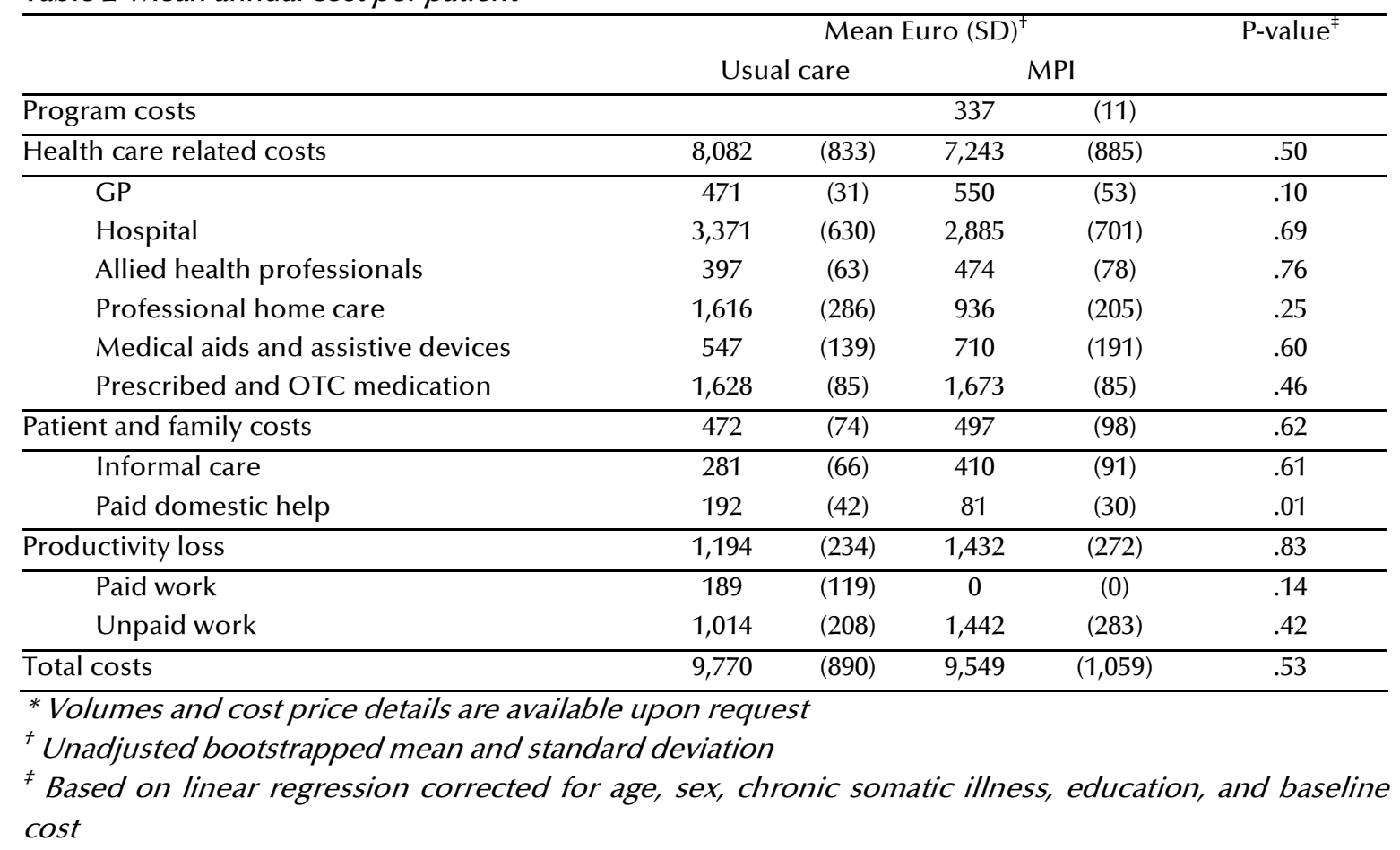




\section{Annual costs and clinical effects}

The control group had slightly higher costs than the intervention group (€ 9,770 vs. € 9,549; Table 2). Hence, a cost saving was achieved in the intervention group, despite the extra costs (on average €337) of the MPI in the intervention group. However, the overall cost difference was not significant, although a significant difference was found in costs of paid domestic help, in favour of the group that received the MPI ( $€ 192$ vs. $€ 81 ; p=.01$ ).

Linear regression of the clinical effects revealed a significant difference between utilities derived from the EQ5D at the final follow-up measurement $(p=.02)$. However, no significant differences between utilities were found at the other follow-up measurements. In addition, no significant differences in mean QALY-EQ5D or mean QALYDFDs were found between patients from the intervention and control groups, although outcomes were all in favour of the intervention group (Table 3).

Table 3 Clinical effects

\begin{tabular}{|c|c|c|c|}
\hline & \multicolumn{2}{|c|}{ Mean $(\mathrm{SD})^{*}$} & \multirow[t]{3}{*}{ P-value ${ }^{t}$} \\
\hline & Usual Care & MPI & \\
\hline & $\mathrm{n}=118$ & $\mathrm{n}=110$ & \\
\hline$\overline{\text { QALY - EQ5D }^{\ddagger}}$ & $0.59(0.02)$ & $0.62(0.02)$ & .06 \\
\hline Utility at three monthsc & $0.61(0.02)$ & $0.64(0.02)$ & .10 \\
\hline Utility at six monthsc & $0.59(0.02)$ & $0.61(0.02)$ & .33 \\
\hline Utility at twelve monthsc & $0.56(0.02)$ & $0.62(0.02)$ & .02 \\
\hline QALY - DFD ${ }^{\S}$ & $0.78(0.01)$ & $0.80(0.01)$ & .31 \\
\hline DFD / yeard & $163(11)$ & $184(12)$ & .31 \\
\hline \multicolumn{4}{|c|}{ *Unadjusted bootstrapped mean and standard deviation } \\
\hline \multirow{2}{*}{\multicolumn{4}{|c|}{$\begin{array}{l}{ }^{+} \text {Based on linear regression corrected for age, sex, chronic somatic illness, education, and baseline } \\
\text { EQ5D or BDI score }\end{array}$}} \\
\hline & & & \\
\hline \multicolumn{4}{|c|}{${ }^{*}$ Based on the Dutch algorithm for EQ5D scores } \\
\hline${ }^{S}$ Based on the BDI scores & & & \\
\hline
\end{tabular}

\section{Cost-utility}

Because patients receiving the MPI had lower costs and experienced greater health effects, the MPI dominated usual care (Table 4). The ICUR of the primary analysis showed a saving of $€ 11,508$ per QALY-EQ5D (95\% Cl -160,502 to 192,027). The ICUR of the secondary analysis showed a saving of $€ 12,534$ per QALY-DFD $(95 \% \mathrm{Cl}-190,366$ to $101,049)$. The cost saving per depression-free day was $€ 14(95 \% \mathrm{Cl}-157$ to 106 ; data not shown). 


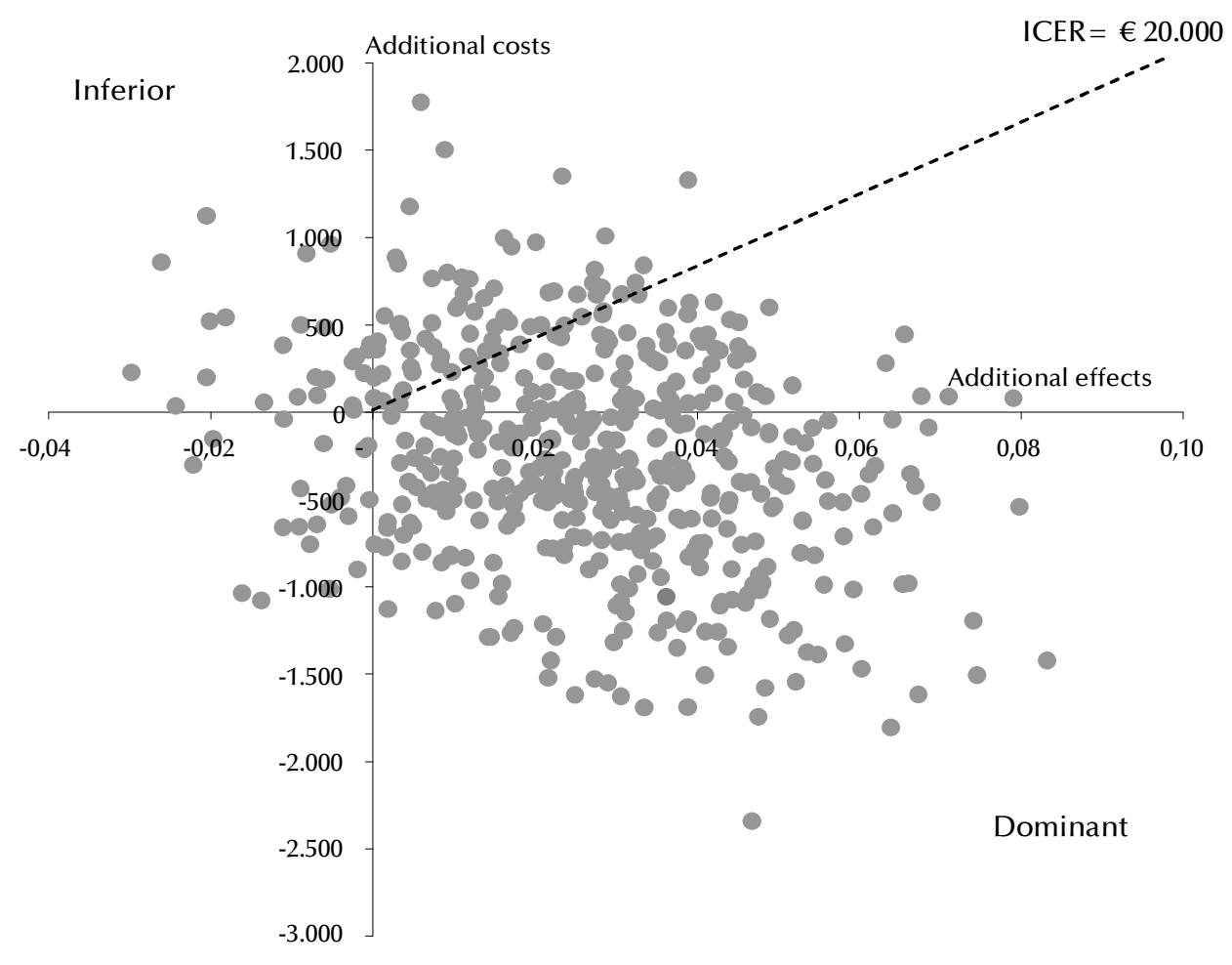

Figure 1 Cost-effectiveness plane: mean cost difference between the MPI and usual care groups (vertical axis) and mean effect difference (horizontal axis).

Bootstrap replications of the ICUR were performed to address the uncertainty surrounding this ratio. The results of the primary analysis, using the QALY-EQ5D, are presented in a cost-effectiveness plane (Figure 1), with differences in cost on the vertical axis and differences in effects on the horizontal axis. Each dot $(n=1,000)$ represents a bootstrap replication. The distribution of our primary analysis shows that $63 \%$ of the dots are in the lower right-hand quadrant, indicating a probability of $63 \%$ that our MPI is the dominant treatment, because the MPI is less costly and more effective than care as usual (see also Table 4). In addition, $28 \%$ of the dots are located in the upper right-hand quadrant, indicating that a health gain is produced, but at additional costs. On the other hand, there is a probability of $5 \%$ that the MPI is inferior (upper left-hand quadrant) and $4 \%$ that the MPI is less costly but also less effective (lower left-hand quadrant). The percentage of dominance for the secondary analysis, based on DFDs, is slightly higher (67\%; Table 4) than that in our primary analysis.

Interpretation of these outcomes also depends on how much decision-makers are willing to pay for each quality-adjusted-life-year gained. For instance, if a decisionmaker is willing to pay $€ 20,000$ per QALY gained, the probability of the MPI being cost-effective is about $82 \%$ (see dotted line in Figure 1 and Figure 2). 


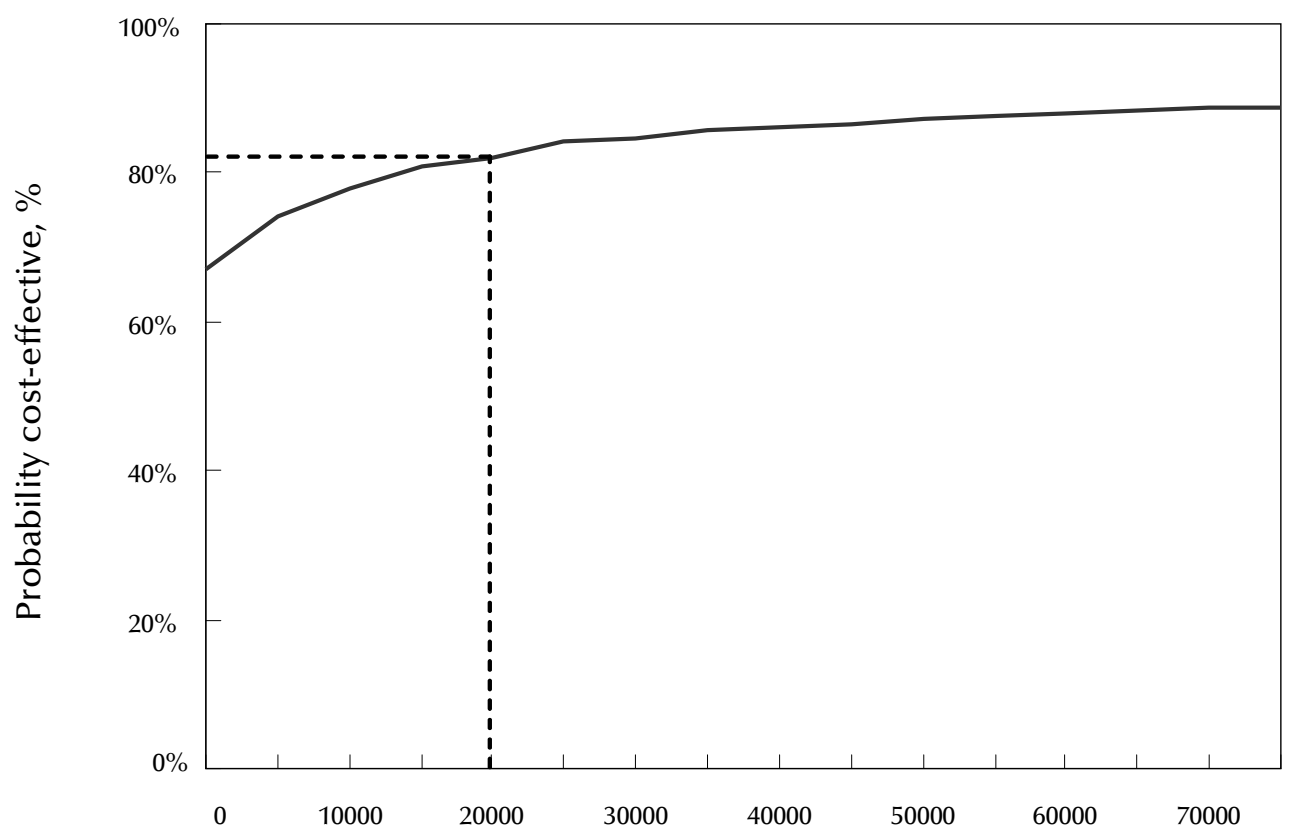

Ceiling ratio for willingness to pay, €/QALY

Figure 2 Cost-utility acceptability curve: probability that the MPI is cost-effective (vertical axis) given various ceiling ratios for willingness to pay (horizontal axis).

In Dutch health care, this ceiling ratio is often considered a reasonable critical level for QALY cost. ${ }^{33}$ A Dutch advisory committee to the Ministry of Health even proposed a ceiling ratio of $€ 80,000$ per QALY gained, ${ }^{34}$ which would result in an $89 \%$ probability of our intervention being superior to care as usual. This is further illustrated in the cost-effectiveness acceptability curve of our primary analysis (Figure 2). The probability of our intervention being superior to usual care ( $y$-axis) is shown for varying ratios for willingness to pay for each quality-adjusted-life-year gained. ${ }^{35} 36$

The sensitivity analyses, both the complete case analysis and the analysis with a reduction of program costs, indicated that results of our primary analysis were robust (Table 4). Both ICURs showed dominance, and the probabilities of the intervention being dominant were largely the same as in our primary analysis. In the complete case analysis, however, the probability of the MPI being less effective and less costly increased, while the probability of the MPI being more costly but also more effective decreased. As expected, a reduction of program costs led to an increase in dominance, since the costs in the intervention group were lower, while the effects remained the same. 


\begin{tabular}{|c|c|c|c|c|c|c|c|}
\hline \multirow{2}{*}{ Type of analysis } & \multicolumn{2}{|c|}{$\mathrm{N}$} & \multirow[b]{2}{*}{ 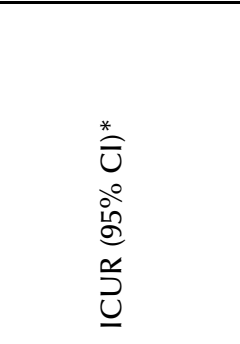 } & \multicolumn{4}{|c|}{ Probability of cost-effective MPI*, $\%$} \\
\hline & $\frac{\frac{0}{0}}{\frac{\pi}{0}}$ & $\overline{\bar{z}}$ & & 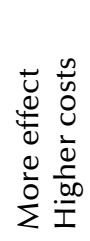 & 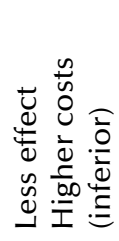 & 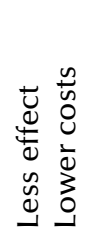 & 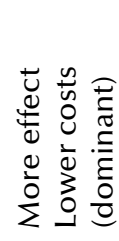 \\
\hline $\begin{array}{l}\text { Primary analysis } \\
\text { QALY - EQ5D }\end{array}$ & 118 & 110 & $\begin{array}{c}\text { Dominance } \\
(-160,502-192,027)\end{array}$ & 28 & 5 & 4 & 63 \\
\hline $\begin{array}{l}\text { Secondary analysis } \\
\text { QALY - DFD }\end{array}$ & 118 & 110 & $\begin{array}{c}\text { Dominance } \\
(-190,366-101,049)\end{array}$ & 29 & 2 & 2 & 67 \\
\hline
\end{tabular}

Sensitivity analyses

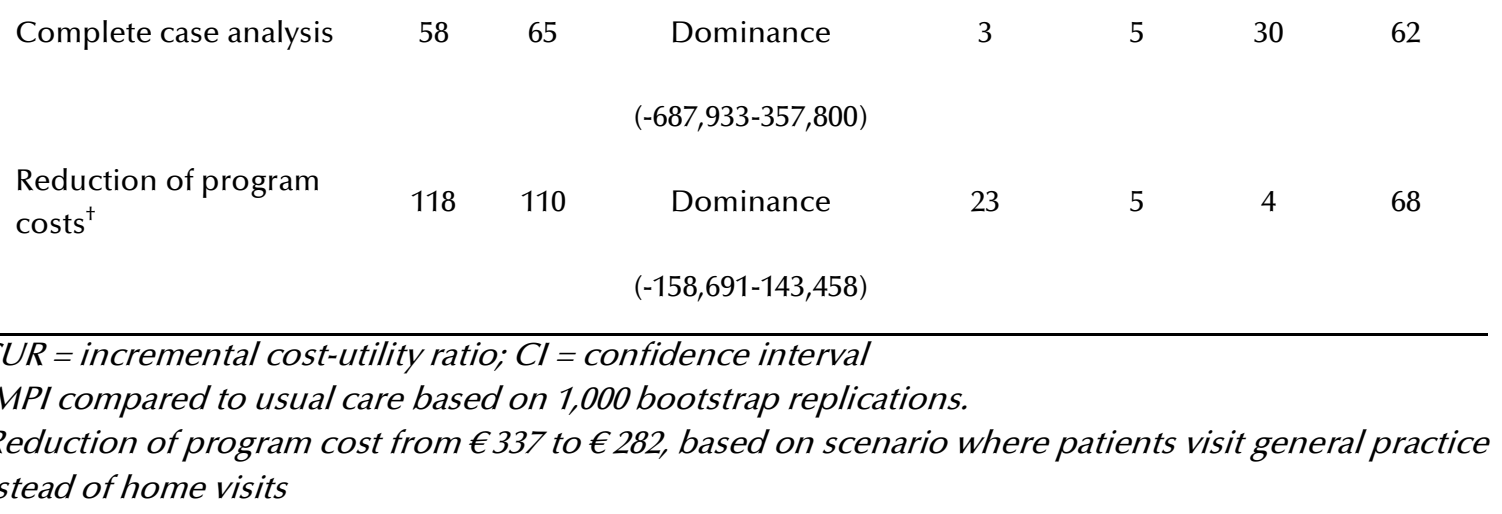

\section{DISCUSSION}

In this group of elderly patients with DM or COPD and co-occurring minor, mild to moderate major depression or dysthymia, our minimal psychological intervention will probably lead to improvements in quality of life and depression-free days, and a decrease in mean annual costs. In our study, the MPI proved to be the dominant treatment, which is supported by the cost-effectiveness acceptability plane showing a $63 \%$ probability that the MPI is less costly and more effective than usual care. If decision-makers are willing to pay $€ 20,000$ per QALY gained, the probability increases to $82 \%$. Sensitivity analyses showed that our findings are robust; the probability of a cost-effective intervention remained largely the same.

Limitations of this study include the attrition rate. This could have influenced our findings, especially because persons who dropped out of the analysis tended to be older and had poorer quality of life at baseline. However, attrition was not significantly different between the intervention and control groups. We used person specific mean imputation techniques to address issues of missing data. This method is considered valid, ${ }^{32}$ although more refined techniques might have provided better 
estimates. ${ }^{37}$ A complete case analysis showed that our imputation techniques may have caused a higher probability of our intervention being cost-effective. A second limitation is the poor validity of the DFD method to establish cost-utility. ${ }^{31}$ Cost-utility analyses have been introduced to provide a generic outcome measure for the comparison of costs and effects across diseases. ${ }^{13}$ However, the cost-utility estimate of the DFD method is not based on generic outcomes but on depression severity, making it invalid for comparison with other diseases than depression. Nevertheless, we decided to include the DFD method as a secondary analysis, to be able to compare our results with those of studies using the DFD method. A third limitation concerns the generalisability of our findings across the two chronic somatic illnesses. We added chronic somatic illnesses to our regression models, but the individual costeffectiveness outcomes for diabetic and COPD patients remain unclear, as our study population was too small for disease-specific analyses. Fourth, we used a cost diary to assess the cost data. This method has some advantages over questionnaires, because it measures healthcare consumption prospectively and might be more accurate. ${ }^{22} 38$ However, cost diaries often have relatively high levels of missing values. Finally, to reduce the burden for the patients, we measured discontinuous. Previous studies revealed that measuring in at least three months during a year provides good estimates of annual costs. ${ }^{22} 39$ Nevertheless, high one-time expenses, such as inpatient hospital stays, might be missed or overestimated by measuring discontinuously. Also, the estimate of the QALY would have been more precise is we had used more measurement times.

While reviewing our results, one should take into account that differences in clinical effects, in terms of QALYs, were not significant between the intervention and control groups. However, the utility scores showed a significant difference at the final followup. An increase in differences over time has also been found for the depression parameter in our effectiveness study. ${ }^{40}$ Since our intervention is a short, cognitive behavioural skill based program, the most plausible explanation would be that patients gained skills to cope with depression and used them more and more in daily life. Therefore, the difference in effect may become more pronounced at longer follow-up. The lack of significant cost differences in our study had been anticipated: the goal of our intervention was not to reduce health care utilization, but to improve the quality of life for patients with a chronic somatic illness. In the long run, however, improvement of the health-related quality of life could lead to a reduction of health care utilization. Our cost analysis shows that the most considerable cost savings were in favour of the intervention group, and a longer follow-up might show a further reduction of health care utilization.

Our study found a cost saving of $€ 14$ per depression-free day. According to a review by Wang et al., most other studies found an additional cost per depression-free day ranging from $€ 7$ to $€ 26$, instead of a cost saving. ${ }^{9}$ However, none of these studies focused on persons with chronic somatic illnesses. Recently, two additional studies were published that focused on depression treatment of persons with DM. ${ }^{4142}$ In line with our findings, these studies found beneficial cost-effectiveness outcomes, 


\section{CHAPTER 7}

although both studies only reviewed health care related costs, not including production losses, and had a follow-up of twenty-four months instead of twelve months.

One of our sensitivity analyses was based on an implementation scenario in which patients visit a GP's surgery to receive the MPI instead of nurses visiting patients at home. In the Netherlands, primary care nurses are increasingly employed in general practice to treat persons with chronic somatic illnesses. Therefore, detection and monitoring of depressive symptoms and, if necessary, depression treatment could easily be integrated in regular care. This scenario seems to be a good alternative to home visits by nurses exclusively providing depression treatment, especially if health care insurers are willing to invest in an improved quality of life for persons with chronic somatic illnesses. Results of our process evaluation, ${ }^{18}$ effectiveness evaluation, ${ }^{40}$ and this cost-utility study support further dissemination of our MPI for elderly persons with a chronic illness.

Our findings suggests that treatment of depression with our minimal psychological intervention in persons with a chronic somatic illness probably leads to improvements in quality of life and depression-free days at no greater cost than care as usual, implying a cost-effective intervention. These results support further dissemination of the MPI, for example in disease management programs for persons with chronic somatic illnesses.

\section{Acknowledgements}

We thank Kitty Daemen, Henny Geelen, Francine Hendriks, and Mieke Witte, for administering the intervention with dedication and enthusiasm, Wendy Engering for her contribution to the patient recruitment, Dorien Mintjes for her contribution to the data entry, as well as MEMIC (Centre for Data and Information Management) for providing us with a data management system. This study was funded by the Netherlands Organisation for Health Research and Development (ZonMw), Health Care Efficiency Research Program, grant number 945-03-047. 


\section{REFERENCES}

1. Djernes JK. Prevalence and predictors of depression in populations of elderly: a review. Acta Psychiatr Scand 2006;113(5):372-87.

2. $\quad$ Spitzer RL, Kroenke K, Linzer M, Hahn SR, Williams JB, deGruy FV, 3rd, et al. Health-related quality of life in primary care patients with mental disorders. Results from the PRIME-MD 1000 Study. JAMA 1995;274(19):1511-7.

3. Penninx BW, Geerlings SW, Deeg DJ, van Eijk JT, van Tilburg W, Beekman AT. Minor and major depression and the risk of death in older persons. Arch Gen Psychiatry 1999;56(10):889-95.

4. Penninx BW, Guralnik JM, Ferrucci L, Simonsick EM, Deeg DJ, Wallace RB. Depressive symptoms and physical decline in community-dwelling older persons. JAMA 1998;279(21):1720-6.

5. Anderson RJ, Freedland KE, Clouse RE, Lustman PJ. The prevalence of comorbid depression in adults with diabetes: a meta-analysis. Diabetes Care 2001;24(6):1069-78.

6. Yohannes AM, Baldwin RC, Connolly MJ. Prevalence of sub-threshold depression in elderly patients with chronic obstructive pulmonary disease. Int. J. Geriatr. Psychiatry 2003;18(5):412-6.

7. DiMatteo MR, Lepper HS, Croghan TW. Depression is a risk factor for noncompliance with medical treatment: meta-analysis of the effects of anxiety and depression on patient adherence. Arch Intern Med 2000;160(14):2101-7.

8. Donohue JM, Pincus HA. Reducing the societal burden of depression: a review of economic costs, quality of care and effects of treatment. Pharmacoeconomics 2007;25(1):7-24.

9. Wang PS, Simon G, Kessler RC. The economic burden of depression and the cost-effectiveness of treatment. Int J Methods Psychiatr Res 2003;12(1):22-33.

10. Pirraglia PA, Rosen AB, Hermann RC, Olchanski NV, Neumann P. Cost-utility analysis studies of depression management: a systematic review. Am J Psychiatry 2004;161(12):2155-62.

11. Gilbody S, Bower P, Whitty P. Costs and consequences of enhanced primary care for depression: systematic review of randomised economic evaluations. Br J Psychiatry 2006;189:297-308.

12. Lamers F, Jonkers CC, Bosma H, Diederiks JP, van Eijk JT. Effectiveness and cost-effectiveness of a minimal psychological intervention to reduce non-severe depression in chronically ill elderly patients: the design of a randomised controlled trial [ISRCTN92331982]. BMC Public Health 2006;6(1):161.

13. Drummond MF. Methods for the economic evaluation of health care programmes. 2nd ed. Oxford: Oxford University Press, 1997.

14. Spitzer RL, Kroenke K, Williams JB. Validation and utility of a self-report version of PRIME-MD: the PHQ primary care study. Primary Care Evaluation of Mental Disorders. Patient Health Questionnaire. JAMA 1999;282(18):1737-44.

15. Sheehan DV, Lecrubier $\mathrm{Y}$, Sheehan KH, Amorim P, Janavs J, Weiller E, et al. The Mini-International Neuropsychiatric Interview (M.I.N.I.): the development and validation of a structured diagnostic psychiatric interview for DSM-IV and ICD-10. J. Clin. Psychiatry1998;59 Suppl 20:22-33.

16. Hamilton M. A rating scale for depression. J. Neurol. Neurosurg. Psychiatry 1960;23:56-62.

17. Van Eijk JT, Diederiks JP, Kempen GI, Honig A, van der Meer K, Brenninkmeijer WJ. Development and feasibility of a nurse administered strategy on depression in community-dwelling patients with a chronic physical disease. Patient Educ. Couns. 2004;54(1):87-94.

18. Jonkers CCM, Lamers F, Bosma H, Metsemakers JF, Kempen GIJM, van Eijk JTM. Process evaluation of a minimal psychological intervention to reduce depression in chronically ill elderly persons. Patient Educ. Couns. 2007;68(3):252-257.

19. Geijer RMM, van Schayk CP, van Weel C, Sachs APE, van der Zwan ACC, Bottema BJAM, et al. NHGStandaard COPD en Astma bij Volwassenen: Behandeling [NHG Practice Guideline COPD: Treatment]. Huisarts Wet 1997;40:430-442.

20. Geijer RMM, Thiadens HA, Smeele IJM, Sachs APE, Bottema BJAM, van Hensbergen W, et al. NHGStandaard COPD en Astma bij Volwassenen: Diagnostiek [NHG Practice Guideline COPD: Diagnosis]. Huisarts Wet 2001;44(3):107-117.

21. Rutten GEHM, De Grauw WJC, Nijpels G, Goudswaard AN, Uitewaal PJM, Van der Does FEE, et al. NHGStandaard Diabetes mellitus type 2 [NHG Practice Guideline Diabetes mellitus type 2]. Huisarts Wet 2006;49(3):137-152. 


\section{CHAPTER 7}

22. Goossens ME, Rutten-van Molken MP, Vlaeyen JW, van der Linden SM. The cost diary: a method to measure direct and indirect costs in cost-effectiveness research. J Clin Epidemio/2000;53(7):688-95.

23. Oostenbrink JB, Bouwmans CAM, Koopmanschap MA, Rutten FFH. Handleiding voor kostenonderzoek: Methoden en standaard kostprijzen voor economische evaluaties in de gezondheidszorg [Manual for costing: Methods and Standard Costs for Economic Evaluations in Health Care]. Diemen: College voor zorgverzekeringen, 2004 updated version.

24. Oostenbrink JB, Koopmanschap MA, Rutten FF. Standardisation of costs: the Dutch Manual for Costing in economic evaluations. Pharmacoeconomics 2002;20(7):443-54.

25. EuroQol G. EuroQol--a new facility for the measurement of health-related quality of life. The EuroQol Group. Health Policy 1990;16(3):199-208.

26. Dolan P. Modeling valuations for EuroQol health states. Med Care 1997;35(11):1095-108.

27. Lamers LM, McDonnell J, Stalmeier PF, Krabbe PF, Busschbach JJ. The Dutch tariff: results and arguments for an effective design for national EQ-5D valuation studies. Health Econ 2006;15(10):1121-32.

28. Matthews JN, Altman DG, Campbell MJ, Royston P. Analysis of serial measurements in medical research. Bmj1990;300(6719):230-5.

29. Beck AT, Steer RA, Garbin MG. Psychometric properties of the Beck Depression Inventory: Twenty-five years of evaluation. Clinical Psychology Rev. 1988;8(1):77-100.

30. Lave JR, Frank RG, Schulberg HC, Kamlet MS. Cost-effectiveness of treatments for major depression in primary care practice. Arch Gen Psychiatry1998;55(7):645-51.

31. Pyne JM, Tripathi S, Williams DK, Fortney J. Depression-free day to utility-weighted score: is it valid? Med Care 2007;45(4):357-62.

32. Engels JM, Diehr P. Imputation of missing longitudinal data: a comparison of methods. J Clin Epidemiol 2003;56(10):968-76.

33. Casparie AF, van Hout BA, Simoons ML. Richtlijnen en kosten [Guidelines and costs]. Ned Tijdschr Geneeskd1998;142(38):2075-7.

34. Council for Public Health and Health Care. Sensible and sustainable care; recommendations produced by the Council for Public Health and Health Care to the Minister of Health, Welfare and Sport. Zoetermeer: Council for Public Health and Health Care, 2006.

35. van Hout BA, Al MJ, Gordon GS, Rutten FF. Costs, effects and C/E-ratios alongside a clinical trial. Health Econ 1994;3(5):309-19.

36. Fenwick E, O'Brien BJ, Briggs A. Cost-effectiveness acceptability curves--facts, fallacies and frequently asked questions. Health Econ 2004;13(5):405-15.

37. Briggs A, Clark T, Wolstenholme J, Clarke P. Missing... presumed at random: cost-analysis of incomplete data. Health Econ 2003;12(5):377-92.

38. van den Brink M, van den Hout WB, Stiggelbout AM, Putter $\mathrm{H}$, van de Velde CJ, Kievit J. Self-reports of health-care utilization: diary or questionnaire? Int J Technol Assess Health Care 2005;21(3):298-304.

39. Lamoureux EL, Chou SL, Larizza MF, Keeffe JE. The reliability of data collection periods of personal costs associated with vision impairment. Ophthalmic Epidemio/2006;13(2):121-6.

40. Lamers F, Jonkers CCM, Bosma H, Kempen GIJM, Meijer JAMJ, Penninx BWJH, et al. The effectiveness of a minimal psychological intervention in chronically ill elderly patients with depression: a randomised trial (the DELTA-study). submitted.

41. Simon GE, Katon WJ, Lin EH, Rutter C, Manning WG, Von Korff M, et al. Cost-effectiveness of systematic depression treatment among people with diabetes mellitus. Arch Gen Psychiatry 2007;64(1):65-72.

42. Katon W, Unutzer J, Fan MY, Williams JW, Jr., Schoenbaum M, Lin EH, et al. Cost-effectiveness and net benefit of enhanced treatment of depression for older adults with diabetes and depression. Diabetes Care 2006;29(2):265-70. 


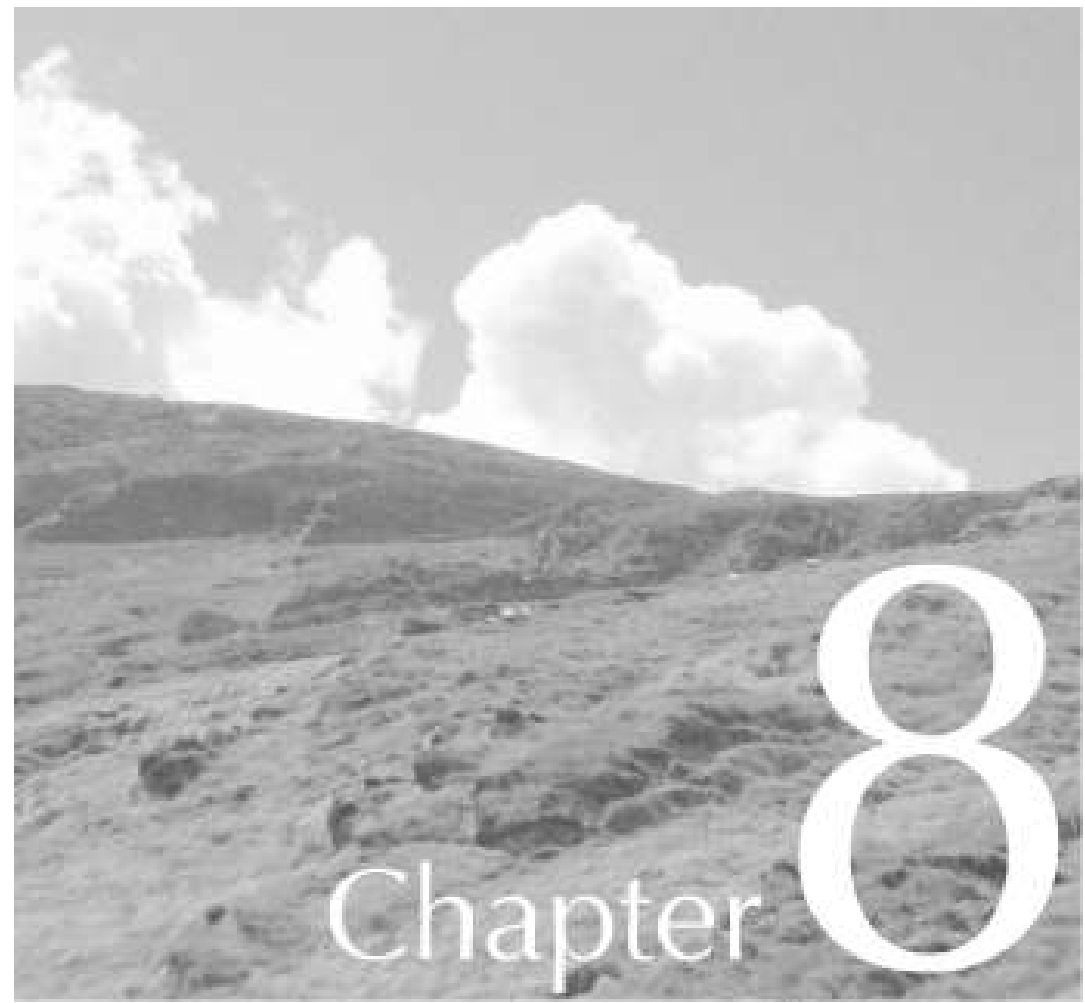

General discussion 
CHAPTER 8 
This thesis described the effectiveness and cost-effectiveness of a nurse-led Minimal Psychological Intervention in reducing depressive symptoms and improving quality of life in chronically ill elderly patients. Furthermore, the validity of a depression screening questionnaire was evaluated. This chapter discusses the main findings of the study, including a reflection on methodological and conceptual considerations, the generalizability of the study results, a comparison with other studies and implications of the findings. Finally, recommendations for future research and an overall conclusion will be formulated.

\section{MAIN FINDINGS}

Regarding the main research question, we found that the MPI was both effective and cost-effective. The MPI was found to have a significant positive effect on depressive symptoms and this effect was found to be generic across DM and COPD, which answers the second research question (Chapter 4). The effect sizes were small to medium and a positive trend of increasing differences between groups over time was observed. The chance of improvement of depressive symptoms, defined as a reduction of baseline depression score with $50 \%$ or more, was thrice as high in the intervention group as in the control group. The MPI also had a significant effect on generic quality of life in diabetic patients at the last follow-up with a medium effect size and with the same pattern of increasing between-group differences over time (Chapter 4). The cost-effectiveness study provided important information for health policy makers and health care insurers and showed that with a probability of $63 \%$, the MPI is less costly and more effective than usual care (Chapter 7). If decision makers would be willing to pay $€ 20,000$ per Quality Adjusted Life Year (QALY) gained, this probability would increase to $82 \%$. A cost saving of $€ 14$ per depression-free day was found.

Besides collecting data on depression and quality of life outcomes, data on several disease-specific outcomes were collected during follow-up. We found that the MPI also had an effect on these disease-specific outcome measures. On a COPD-specific quality of life measure, we found significant effects in favour of the intervention, with medium effect-sizes. Effects were largest on the Impact subscale, which covers social functioning and psychological disturbances resulting from COPD, and again, between-group differences increased over time. Anxiety is also common in COPD patients. The intervention had a significant effect on symptoms of anxiety in COPD patients; control patients on average had more symptoms of anxiety than intervention patients during follow-up (Chapter 6). In diabetic patients, significant effects on diabetes-specific quality of life were only found in certain subgroups (Chapter 5). Higher educated patients, male patients and patients with a shorter duration of diabetes benefited from the intervention. Hba1c levels, an indicator of glycemic control, of patients receiving the intervention decreased over time, indicating a better glycemic control. In control patients, an opposite pattern was observed in Hba1c levels, and this difference in trend between groups was statistically significant. 
In the study evaluating the validity and reliability of the Patient Health Questionnaire9 (PHQ-9) depression screening questionnaire (Chapter 3), we found that the PHQ-9 was a reliable instrument in elderly with a chronic somatic disease. The validity of two scoring methods for the PHQ-9 was evaluated. The first method was the algorithmbased score, which is a dichotomous outcome based on the DSM-IV criteria for depression (at least 5 symptoms and at least one core symptom present). The second method was the summed score, which sums the score of all nine items and ranges from 0 to 27. The validity of this summed PHQ-9, using the Mini International Neuropsychiatric Interview (MINI) as reference, was found to be good. The algorithm-based PHQ-9 score on the other hand, had low sensitivity meaning that it missed a high percentage of depressed cases. Low sensitivity was due to the underreporting of the two core symptoms of depression on the PHQ-9, namely depressed mood and loss of interest or pleasure. The fact that patients felt reluctant to report these psychological symptoms, but not to report somatic symptoms, may indicate that the fear of being stigmatized is still present in elderly populations.

Overall, with respect to the research questions, we have shown that the MPI is effective in reducing depressive symptoms and improving quality of life in chronically ill elderly, with consistent patterns of increasing differences over time. This supports the theory that over time, patients in the intervention group continued to use the skills they had learned in the intervention in their daily lives, leading to less depressive symptoms in the long-term. Furthermore, there is a high probability that the intervention does not cost more than care as usual, but even leads to a cost saving, and is therewith cost-effective. It can therefore be concluded that the MPI seems well suited for implementation within regular care and existing disease-management programmes, improving the care for chronically ill elderly persons.

\section{METHODOLOGICAL AND CONCEPTUAL CONSIDERATIONS}

\section{Selection and attrition bias}

Bias in trials may occur as a result of non-response or loss to follow up. ${ }^{1}$ Bias may affect the external validity and consequently the generalisability of the findings, or affect internal validity which may lead to an over- or underestimation of the effect of the intervention. In our study, participants in the diagnostic interview (MINI) and non-participants did not differ significantly on demographic characteristics or PHQ-9 depression score, so chances of a potential bias at this stage seem unlikely. Persons who participated in the DELTA study however, were younger and significantly higher educated than eligible persons who refused to participate. This may have led to selection bias with an overrepresentation of higher educated and younger persons; the consequences of this will be discussed in a later section.

Attrition may lead to an imbalance in characteristics of groups, threatening the internal validity, and may limit the external validity when attrition is selective. It is 
therefore important to take measures to prevent attrition or missingness of data and to choose proper methods of analyses. In the DELTA-study, efforts were taken to keep patients in the study. Patients who did not wish to receive the intervention or discontinued the intervention were encouraged to continue to fill in the follow-up questionnaires or at least fill in the final follow-up questionnaire. Control patients who wanted to stop participating in the trial were likewise encouraged to fill in the last questionnaire. Attrition rates up to $30 \%$ are not uncommon in populations of elderly with a chronic disease and increasing age is associated with attrition. ${ }^{23}$ In the DELTAstudy, approximately $33 \%$ of the participants dropped out. Attrition bias may have occurred as drop-outs were significantly older than those remaining in the study and this may have affected external validity. The variables age, gender and educational level were standard included in the models to reduce the chance of bias caused by imbalances in these variables between groups. Furthermore, the use of mixed-effects models in the data analyses may have reduced the influence of attrition bias, as this technique uses all available data. It does not delete persons with missing observations, nor does it imputes measurements. ${ }^{4}$ It therefore thought to be the preferable method in the analysis of repeated measurements and is valid under the missingness mechanism of missing at random (MAR), which is thought to be a reasonable assumption in clinical trials and was assessed likely to be the case in our study. ${ }^{56}$

\section{MTA methodology}

Outcomes from MTA research are an important information source in the decisionmaking processes of policymakers and health care insurers. With methodology stemming from economic theories, this relatively young research area is a constantly developing one. Especially in terms of statistical methods, much has improved over the last decade. ${ }^{7}$

In our cost-effectiveness study, we used both quality-adjusted-life-years (QALYs) and depression-free days (DFDs) as outcome measures for the effects of the MPI. Over the last few years, the DFDs method as outcome measure of effect is increasingly used in depression research. ${ }^{8-11}$ However, the use of this DFD method is under discussion. ${ }^{12}$ Normally, the outcome measure for the effects of a treatment is a generic outcome. This allows for the comparison with other treatments and conditions, which is important for decision makers in health care. The DFD method is not a generic outcome and results can therefore only be compared with other depression treatment studies using this method. DFDs can further be used to estimate QALYs. In theory, these DFD-QALYs can be used for comparison with other treatments and conditions. However, as long as the validity of the method used to calculate DFDs and QALYs from DFDs remains unclear, it is not advisable to compare DFD-based QALYs with QALYs based on generic outcomes. Despite the concerns about the validity of the DFD-method, we decided to include DFDs to make comparison with other studies using the DFD-method possible. 


\section{Minor depression: to treat or not to treat}

Minor depression is increasingly thought to be on a continuum with major depression. ${ }^{13}{ }^{14}$ Epidemiological studies have supported the view that depression is a dynamic disorder, evolving on a continuous scale. ${ }^{1516}$ Studies have further shown that patients with minor depression are at higher risk of developing major depression compared to non-depressed persons, ${ }^{17} 18$ and spontaneous remission rates of minor depression are low. ${ }^{19}$ In light of preventive strategies, it would have been interesting to evaluate whether the MPI can prevent new cases of major depression. In our study, however, we were not able evaluate whether the intervention had a preventive effect, as we did not use a diagnostic depression instrument in the data collection at followup. Nevertheless, intervention patients had a threefold higher chance of improving their depression severity with $50 \%$ or more compared to baseline than controls. Since the intervention seems to have a strong effect on the reduction of depressive symptoms, a preventive effect on developing major depression seems likely.

The notion of a continuum of depression has also received criticism; where does everyday unhappiness end and where does a clinical disorder begin? In light of the 'medicalisation of unhappiness' the need of treatment of minor depression is often questioned. By accepting minor depression as a disorder, we run the risk of overtreating persons. Especially with antidepressant treatments the treatment may be worse than the condition itself, given the chance of side effects and, in chronically ill elderly, the possible interaction with other medication. ${ }^{20}$ Nevertheless, the number of studies evaluating antidepressants for minor depression increases steadily. ${ }^{21-26}$ Further, in the late nineties, GPs often prescribed antidepressants in less severe cases of depression where the use of antidepressants was not indicated by clinical guidelines. ${ }^{27}$ Another problem with starting active treatment in minor depression is the stigmatization that persons may experience when they are labelled as having minor depression. It is known that patients, in reaction to expected discrimination, remain secretive about their condition and treatment or withdraw from social life. This may have negative consequences for their social support network and self-esteem. ${ }^{28}$ In clinical guidelines, ${ }^{2930}$ minor depression currently is not included and in current literature, watchful waiting strategies or activating patients into pleasant activities are proposed as suitable strategies for minor depression. ${ }^{23} 31$ Watchful waiting only requires action from the GP and activation can be presented to the patient as a general advice without labelling the patient as minor depressive. These strategies seem sensible, since both approaches are non-invasive, and do not involve labelling and stigmatisation. However, in some cases of minor depression, more active forms of treatment seem feasible and advisable. As our study showed, patients who are at high risk of developing major depression, like persons with chronic somatic diseases, may benefit from minimal strategies aiming to improve coping and self-management skills. These interventions can be introduced as skill-enhancing, can be incorporated within disease management programs, and do not necessary need to be labelled as 'depres- 
sion treatment', which reduces the chance of stigmatisation of patients receiving such treatment.

\section{GeNERALIZABILITY}

The DELTA study was designed as a pragmatic randomised controlled trial. In a pragmatic trial, the effectiveness of a new treatment in everyday clinical practice is evaluated. ${ }^{32}$ Inclusion and exclusion criteria are kept to a minimum to assure good external validity. A relatively heterogeneous study population forms a representative sample of patients that would be considered for the treatment in routine primary care. A downside to pragmatic trials is that the effort to ensure external validity may be at the expense of internal validity. ${ }^{33}$ For instance, in pragmatic trials, patients are not always blinded and this may introduce bias. In our study, certain measures were taken to ensure internal validity; randomisation was performed by an external agency, blinded for the researchers and during data entry researchers were not aware of treatment allocation.

Even though the pragmatic character of the DELTA-study ensures high external validity, there are limitations to the generalizability of the results. In our sample, younger and higher educated patients were somewhat overrepresented due to attrition and selection bias. As previously mentioned, patients with a lower education potentially experience less benefit from the intervention. ${ }^{34}$ This means that in daily practice, where the population on average is lower educated than the population in the trial, the effects of the MPI overall may be lower than the effects observed in the trial. Furthermore, we did only include persons with diabetes and COPD, and although the effect of the intervention on depressive symptoms was comparable, we can not blindly assume that it will be as effective in other chronic disease as well. However, others have shown that the type of chronic disease does not influence treatment outcome. ${ }^{35-37}$ Furthermore, many self-management approaches advocate the generality of their program. For instance, Lorig's Chronic Disease SelfManagement Program (CDSMP) is given in mixed groups with the underlying assumption that persons with a chronic disease all face the same adaptive tasks. ${ }^{38}$ Also, data from the process evaluation showed that patients who received MPI in our study would recommend the intervention to patients with other chronic diseases as well. ${ }^{39}$ These findings support the view that the MPI is likely to be successful in patients with other chronic diseases as well.

In the current cost-effectiveness analyses, costs of screening, diagnosing and monitoring patients were not included. Although the results of the cost-effectiveness study are favourable, with a high probability of a cost-effectiveness intervention, the costsaving found in our study will be lower in real life settings, as it will take time and thus money to screen, diagnose and monitor patients. 


\section{FINDINGS DELTA IN LIGHT OF PREVIOUS FINDINGS}

In the Netherlands, several studies have evaluated (short) psychological treatments compared to usual care in primary care settings. A study that included patients with minor depression found that minimal-contact psychotherapy reduced depressive symptoms and lowered the incidence of major depression. ${ }^{40}$ Other studies focusing on major depression or mental health problems, found that psycho-educational prevention plus CBT had an effect on depressive symptoms in major depression, ${ }^{41}$ while interpersonal psychotherapy and nurse-administered problem-solving therapy only seemed to be effective in more severe cases of major depression and mental health problems. ${ }^{42} 43$

In recent years, many depression trials have focused on collaborative care (e.g. IMPACT, PROSPECT, PATHWAYS). ${ }^{44-46}$ Collaborative care interventions are multifaceted organizational interventions aimed at major depression and involve the collaboration of a general practitioner, case manager and a mental health specialist, and often use algorithm-stepped depression care. Meta-analysis has shown that collaborative care is more effective than standard care, with a mean effect size of 0.31 at twelve months. ${ }^{47}$ Cost-effectiveness studies have shown that collaborative care costs $€ 7$ to $€ 26$ per depression-free day, ${ }^{48}$ and two studies in diabetes patients also found cost savings, but only after 24 months. ${ }^{119}$ In comparison, the intervention in the DELTAstudy had an effect size of 0.29 at approximately 12 months after inclusion and showed a cost saving of $€ 14$ per depression-free-day over a 12 month period. However, when comparing, findings, one should keep the differences between the DELTA study and collaborative care studies in mind. The collaborative care studies were aimed at major depression while we aimed our intervention at patients with minor depression and mild-to moderate major depression. Further, different costperspectives were used. We used a societal perspective, which is much broader than the payers' perspective used in collaborative care studies. The contrast between the treatment arms was also probably different in these studies than in our study, as there are likely to be differences in the standard of usual care, access to care and number of patients that is being treated according to clinical guidelines between the Netherlands and the United States. Also, in the DELTA study, physicians were not notified of participants' depression status, whereas in IMPACT, PROSPECT and PEARLS, physicians were informed. The fact that in DELTA, improvement rates in the control group were only 10 percent, may have been caused by the fact that GPs did not recognise or treat depression in these patients during follow-up. Nevertheless, taking into account the differences between studies, our results are certainly remarkable, considering that collaborative care is an organisational intervention that is usually offered for a longer time period (e.g. IMPACT offered the improved care for 12 months), and our minimal intervention was a patient-aimed intervention with on average four one-hour contacts.

In a recent meta-analysis of treatment for late-life depression, CBT was found to have an effect size of $0.88,{ }^{50}$ which is much larger than our findings. However, differences in setting, duration and intensity of CBT may account for the discrepancy between 
DELTA results and result from this meta-analysis. The cost-effectiveness of psychological treatments for depression, like CBT and problem-solving therapy, has not yet been fully be established, although some studies have suggested that nurses can provide cost-effective treatments. ${ }^{51}$ Our cost-effectiveness results add to the body of evidence of the cost-effectiveness of nurse-administered treatments.

\section{IMPLICATIONS AND RECOMMENDATIONS}

Results from the validation study of the PHQ-9 point to an underreporting of depressive symptoms, especially the core symptoms of depression (depressed mood and loss of interest). This implicates that stigma on depression may still be very much alive in older persons. Patients often present only the somatic symptoms of depression and these somatising persons are less willing to mention psychological symptoms. ${ }^{52} 53$ Education can reduce the stigma on depression in older persons and should be directed to patients as well as and their relatives. It should be made clear that depression is a disorder and not a result of personal failure. There is however, also the side of the GP to consider. It is known that GPs sometimes perceive late-life depression as a natural consequence of ageing and having chronic conditions and therefore do not initiate treatment. Education may be well in place here to bring down these prejudices. But GPs may also make errors in the diagnostic process. Especially in the presence of a chronic somatic disease, GPs may feel reluctant to ascribe somatic symptoms to a depressive disorder. However, research showed that is it not advisable to discount somatic symptoms in the diagnosis of depressive disorder in older persons with somatic diseases. ${ }^{54}$ On the other hand, diagnosing depression is not just the mere counting of symptoms. Assessing the impact of symptoms on daily functioning (social functioning, occupational functioning etc.) is as important as knowing whether symptoms are present. Nevertheless, symptom checklists like the (summed) PHQ-9 may serve as a first filter in the diagnostic process and may facilitate health care professionals in making a depression diagnosis.

In the DELTA study, we did not only find positive results for the effectiveness and cost-effectiveness of the MPI, but also in the process evaluation. Results from this extensive process evaluation showed that both patients and nurses were highly satisfied with the intervention. ${ }^{39}$ This accumulation of evidence supports the implementation of the intervention in the regular care for diabetes and COPD. However, before actions for implementation can be taken, certain matters need to be resolved. For instance, the population at risk needs to be defined, a protocol to detect patients with depressive symptoms and a monitoring system for patients need to be developed, the training and certification of nurses needs to be professionalised, and possible adaptations to the programme need to be explored. First, to which group of patients will the intervention be offered to in primary care? The current study focused on elderly patients, but younger patients $(<60$ years) struggling with the consequences of their disease may benefit from the intervention as well. Also, patients with chronic diseases other than diabetes or COPD may benefit from the intervention. It would be recommended to start implementation for elderly diabetic and COPD 
patients within regular care and disease management programmes, and include younger patients when experiences are positive. In a later stadium, the MPI may then be introduced to disease management programs for other conditions. Second, how are patients with depressive symptoms identified? Given the underreporting of core symptoms of depression, the summed PHQ-9 score would be useful for depression screening in chronically ill elderly patients. In patients with a positive screening outcome, the symptoms and impact on daily functioning should be checked by the nurse, and medical causes like hypothyroidism ruled out, which may imply that patients need a consultation with their GP before the nurse can start administering the MPI. Other criteria need to be protocolised as well; rules for referral to specialized care of patients with suicidal ideation, major psychiatric comorbidity or cognitive decline need to be formed. Third, a monitoring system should be developed with clear guidelines regarding the frequency and manner of monitoring depressive symptoms after conclusion of the intervention, and on what to do in case of nonresponse to treatment or deterioration. It would be advisable for nurses to include the topic of depressive feelings in every subsequent visit of the patient to the clinic. When a patient seems to have deteriorated in terms of depressive symptoms, one or two booster-sessions can be offered, in consultation with the patient and, if necessary, the GP. If a depression becomes severe or a patient develops suicidal ideations, direct referral to the GP or specialized mental health care should take place. Fourth, the training for nurses to administer the intervention should be professionalised. The intervention demands from nurses a change in their attitude towards patients; they are, for instance, not supposed to provide a direct solution to a patients' problem but rather encourage patients explore possible solutions themselves. Not all nurses will be capable of making this attitude change. Therefore, requirements which nurses should meet before receiving a certificate need to be defined. Training and certification should preferably be done through universities of professional education, for example by offering specialized learning modules in the official nursing curriculum. Also, revision and booster sessions need to be organized for nurses working with the intervention. Finally, the results from the diabetic population indicate that not all diabetics benefit equally from the intervention in terms of disease-specific outcomes. Only men, patients with a diabetes duration $<7$ years and higher educated persons showed benefit from the intervention in terms of diabetes-related emotional distress and diabetes symptom burden. Also, results from DELTA-data and a study of Gum et al., indicated that lower educated patients may not benefit equally from psychological interventions as their higher educated counterparts. ${ }^{34} 55$ Areàn and colleagues reported that care managers in a depression treatment trial noted that persons with a lower income required more case management services (referrals to social services, acquiring transportation) than persons with a higher income, suggesting that different groups of patients may want to address different problems. ${ }^{56}$ The differential treatment effect that was found in the DELTA study may be explained by differences in the type of problems that patient addressed and possibly the ability of patients to project the learned skills to other domains of their lives. For instance, if patients have more 
difficulties in finding their way within the health care system or have difficulties in providing themselves with basic needs (housing, transportation), they may want to address these issues first. Since our MPI is very minimal, problems arising from consequences of their disease may not have been addressed during the intervention. Patients with a lower ability to project may be unaware of the possibilities to apply their skills to other domains and this may limit the beneficial effects of the intervention. It would be interesting to determine whether there is indeed a difference in the type and domain of problems that are addressed during the MPI between persons who benefited and those who did not. Future research evaluating interventions with a problem-solving component should collect data on the type of problems that was addressed, as this will provide answers to which kind of adaptations can be made to the intervention in order to achieve maximum benefit in these groups.

The intervention evaluated in the DELTA-study was based on cognitive behavioural therapy (CBT) and self-management. By teaching patients how to deal with the consequences of their chronic disease, their coping skills and level of self-efficacy and mastery are thought to increase. These improved skills will improve their daily functioning and will also affect depressive symptomatology. Although we have shown that depressive symptoms were reduced by the intervention, we yet need to determine what the influence of the MPI was on coping, self-efficacy and mastery, and to which extent these factors mediated the effect of the intervention on depressive symptoms.

Evaluating the effect of depression treatment on the course of a chronic disease may be complicated, as the relationship between chronic disease and depression is a complex one. Although in the last few years, many studies have focused on depression treatment in chronically ill persons, much of the interacting effects remain unknown. It is thought that chronic somatic illness may cause a worsening of depression through the functional impairments associated with the somatic disease. It may also worsen depression through indirect pathophysiologic effects on the brain (via inflammatory markers). ${ }^{57}$ Depression in turn may influence the chronic disease through behaviours (non-adherence to treatment) but also through biological pathways. Depression itself is associated with chronic inflammatory changes; depression enhances the production of pro-inflammatory cytokines. This may aggravate or induce chronic diseases. ${ }^{57}$ Since low-grade inflammation plays a role in both chronic diseases and depression, it would be worthwhile to evaluate to which extent depression treatments can influence inflammatory markers and to which extent this affects the chronic illness as well. Further, as effective depression treatment may improve treatment adherence, disease-specific measures are interesting outcome measures in trials with chronically ill persons. Next to evaluating adherence to treatment regimens and self-care behaviours, measures like symptoms burden, the number of complications over time and disease-specific biomarkers (e.g. HbA1c) can provide a valuable insight to the extent to which depression treatments have a preventive effect on the worsening of the chronic disease. Studies with a longer follow-up are needed to study these potential long-term effects. 


\section{CONCLUSION}

Depression is a highly prevalent and disabling disorder which can have strong negative consequences for elderly with chronic diseases. However, it is often not recognised or treated in primary care.

Findings from the DELTA-study indicate that the PHQ-9 summed score in the screening for depression may serve as a first filter to identify chronically ill elderly with a depression. Skill-enhancing minimal strategies like our Minimal Psychological Intervention (MPI) can then be offered to persons with non-severe cases of major depression and minor depression.

The MPI that was evaluated in the DELTA-study appeared an effective, cost-effective and feasible treatment for diabetic and COPD patient with co-occurring minor depression or mild to moderate major depression. The intervention also had a positive effect on COPD and diabetes-specific outcomes. As nurses were able to administer this minimal strategy successfully, adding the MPI to regular care and existing disease-management programs is likely to lead to improvements in the care for chronically ill elderly persons and to improvements in the patient's health status.

Before implementation activities can be started, protocols for screening, initiating treatment and monitoring of patients need to be formed. Also, the training and certification for the MPI should be included in the course offerings for nurses.

Future research should focus on long-term effects of depression treatment on the course of chronic illnesses and on getting more insight in the interactions between depression and chronic diseases and the contribution of inflammatory markers therein. 


\section{REFERENCES}

1. Kleinbaum DG. Epidemiologic methods: the "art" in the state of the art. J Clin Epidemiol 2002;55(12):1196200.

2. Gardette V, Coley N, Toulza O, Andrieu S. Attrition in geriatric research: how important is it and how should it be dealt with? J Nutr Health Aging 2007;11(3):265-71.

3. Chatfield MD, Brayne CE, Matthews FE. A systematic literature review of attrition between waves in longitudinal studies in the elderly shows a consistent pattern of dropout between differing studies. J Clin Epidemiol 2005;58(1):13-9.

4. Leon AC, Mallinckrodt CH, Chuang-Stein C, Archibald DG, Archer GE, Chartier K. Attrition in randomized controlled clinical trials: methodological issues in psychopharmacology. Biol Psychiatry 2006;59(11):10015.

5. Gueorguieva R, Krystal JH. Move over ANOVA: progress in analyzing repeated-measures data and its reflection in papers published in the Archives of General Psychiatry. Arch Gen Psychiatry 2004;61(3):310-7.

6. Beunckens C, Molenberghs G, Kenward MG. Direct likelihood analysis versus simple forms of imputation for missing data in randomized clinical trials. Clin Trials 2005;2(5):379-86.

7. Doshi JA, Glick HA, Polsky D. Analyses of cost data in economic evaluations conducted alongside randomized controlled trials. Value Health 2006;9(5):334-40.

8. Lave JR, Frank RG, Schulberg HC, Kamlet MS. Cost-effectiveness of treatments for major depression in primary care practice. Arch Gen Psychiatry 1998;55(7):645-51.

9. Katon WJ, Schoenbaum M, Fan MY, Callahan CM, Williams J, Jr., Hunkeler E, et al. Cost-effectiveness of improving primary care treatment of late-life depression. Arch Gen Psychiatry 2005;62(12):1313-20.

10. Lynch FL, Hornbrook M, Clarke GN, Perrin N, Polen MR, O'Connor E, et al. Cost-effectiveness of an intervention to prevent depression in at-risk teens. Arch Gen Psychiatry 2005;62(11):1241-8.

11. Simon GE, Katon WJ, Lin EH, Rutter C, Manning WG, Von Korff M, et al. Cost-effectiveness of systematic depression treatment among people with diabetes mellitus. Arch Gen Psychiatry 2007;64(1):65-72.

12. Pyne JM, Tripathi S, Williams DK, Fortney J. Depression-free day to utility-weighted score: is it valid? Med Care 2007;45(4):357-62.

13. Rowe SK, Rapaport MH. Classification and treatment of sub-threshold depression. Curr Opin Psychiatry 2006;19(1):9-13.

14. Kroenke K. Minor depression: midway between major depression and euthymia. Ann Intern Med 2006;144(7):528-30.

15. Kessing LV. Epidemiology of subtypes of depression. Acta Psychiatr Scand Suppl 2007(433):85-9.

16. Lyness JM, Heo M, Datto CJ, Ten Have TR, Katz IR, Drayer R, et al. Outcomes of minor and subsyndromal depression among elderly patients in primary care settings. Ann Intern Med 2006;144(7):496-504.

17. Cuijpers $P$, de Graaf R, van Dorsselaer S. Minor depression: risk profiles, functional disability, health care use and risk of developing major depression. J Affect Disord 2004;79(1-3):71-9.

18. Cuijpers $P$, Smit F. Subthreshold depression as a risk indicator for major depressive disorder: a systematic review of prospective studies. Acta Psychiatr Scand 2004;109(5):325-31.

19. Hegel MT, Oxman TE, Hull JG, Swain K, Swick H. Watchful waiting for minor depression in primary care: remission rates and predictors of improvement. Gen Hosp Psychiatry 2006;28(3):205-12.

20. Spina E, Scordo MG. Clinically significant drug interactions with antidepressants in the elderly. Drugs Aging 2002;19(4):299-320.

21. Paykel ES, Hollyman JA, Freeling P, Sedgwick P. Predictors of therapeutic benefit from amitriptyline in mild depression: a general practice placebo-controlled trial. J Affect Disord 1988;14(1):83-95.

22. Katon W, Robinson P, Von Korff M, Lin E, Bush T, Ludman E, et al. A multifaceted intervention to improve treatment of depression in primary care. Arch Gen Psychiatry 1996;53(10):924-32.

23. Williams JW, Jr., Barrett J, Oxman T, Frank E, Katon W, Sullivan M, et al. Treatment of dysthymia and minor depression in primary care: A randomized controlled trial in older adults. JAMA 2000;284(12):1519-26.

24. Judd LL, Rapaport MH, Yonkers KA, Rush AJ, Frank E, Thase ME, et al. Randomized, placebo-controlled trial of fluoxetine for acute treatment of minor depressive disorder. Am J Psychiatry 2004;161(10):1864-71. 


\section{CHAPTER 8}

25. Hermens ML, van Hout HP, Terluin B, Ader HJ, Penninx BW, van Marwijk HW, et al. Clinical effectiveness of usual care with or without antidepressant medication for primary care patients with minor or mildmajor depression: a randomized equivalence trial. BMC Med 2007;5(1):36.

26. Brenes GA, Williamson JD, Messier SP, Rejeski WJ, Pahor M, Ip E, et al. Treatment of minor depression in older adults: a pilot study comparing sertraline and exercise. Aging Ment Health 2007;11(1):61-8.

27. Spies TH, Mokkink HGA, de Vries Robbé P, Grol RPTM. Huisarts kiest vaak voor antidepressiva onafhankelijk van de ernst van de depressie. Huisarts Wet 2004;47(8):364-367.

28. Link BG, Cullen FT, Struening E, Shrout PE, Dohrenwend BP. A modified labeling theory approach to mental disorders: An empirical assessment. American Sociological Review 1989;54(3):400-423.

29. National Institute for Clinical Excellence. Depression. Management of depression in primary and secondary care. London: NHS, NICE, 2004.

30. van Marwijk HWJ, Grundmeijer HGLM, Bijl D, van Gelderen MG, de Haan M, van Weel-Baumgarten EM, et al. NHG-standaard Depressieve stoornis (depressie) (eerste herziening). Huisarts Wet 2003;46(11):61423.

31. Whooley MA, Simon GE. Managing depression in medical outpatients. N Engl J Med 2000;343(26):1942-50.

32. Hotopf M. The pragmatic randomised controlled trial. Adv Psychiatr Treat 2002;8(5):326-333.

33. Godwin M, Ruhland L, Casson I, MacDonald S, Delva D, Birtwhistle R, et al. Pragmatic controlled clinical trials in primary care: the struggle between external and internal validity. BMC Med Res Methodol 2003;3:28.

34. Bosma H, Lamers F, Jonkers CCM, van Eijk JTM. Self-management: the holy grail for all or for some only? A randomised controlled trial.

35. Harpole LH, Williams JW, Jr., Olsen MK, Stechuchak KM, Oddone E, Callahan CM, et al. Improving depression outcomes in older adults with comorbid medical illness. Gen Hosp Psychiatry 2005;27(1):4-12.

36. Simon GE, Von Korff M, Lin E. Clinical and functional outcomes of depression treatment in patients with and without chronic medical illness. Psychol Med 2005;35(2):271-9.

37. Bogner HR, Cary MS, Bruce ML, Reynolds CF, 3rd, Mulsant B, Ten Have T, et al. The role of medical comorbidity in outcome of major depression in primary care: the PROSPECT study. Am J Geriatr Psychiatry 2005;13(10):861-8.

38. Lorig KR, Sobel DS, Stewart AL, Brown BW, Jr., Bandura A, Ritter P, et al. Evidence suggesting that a chronic disease self-management program can improve health status while reducing hospitalization: a randomized trial. Med Care 1999;37(1):5-14.

39. Jonkers CCM, Lamers F, Bosma H, Metsemakers JF, Kempen GIJM, van Eijk JTM. Process evaluation of a minimal psychological intervention to reduce depression in chronically ill elderly persons. Patient Educ. Couns. 2007;68(3):252-257.

40. Willemse GR, Smit F, Cuijpers P, Tiemens BG. Minimal-contact psychotherapy for sub-threshold depression in primary care. Randomised trial. Br J Psychiatry 2004;185:416-21.

41. Conradi HJ, de Jonge $\mathrm{P}$, Kluiter $\mathrm{H}$, Smit A, van der Meer $\mathrm{K}$, Jenner JA, et al. Enhanced treatment for depression in primary care: long-term outcomes of a psycho-educational prevention program alone and enriched with psychiatric consultation or cognitive behavioral therapy. Psychol Med 2007;37(6):849-62.

42. Schreuders B, van Marwijk H, Smit J, Rijmen F, Stalman W, van Oppen P. Primary care patients with mental health problems: outcome of a randomised clinical trial. Br J Gen Pract 2007;57(544):886-91.

43. van Schaik A, van Marwijk H, Ader H, van Dyck R, de Haan M, Penninx B, et al. Interpersonal psychotherapy for elderly patients in primary care. Am J Geriatr Psychiatry 2006;14(9):777-86.

44. Bogner HR, Morales KH, Post EP, Bruce ML. Diabetes, depression, and death: a randomized controlled trial of a depression treatment program for older adults based in primary care (PROSPECT). Diabetes Care 2007;30(12):3005-10.

45. Hunkeler EM, Katon WJ, Tang L, Williams JW, Jr., Kroenke K, Lin EH, et al. Long term outcomes from the IMPACT randomised trial for depressed elderly patients in primary care. BMJ 2006.

46. Katon WJ, Von Korff M, Lin EH, Simon G, Ludman E, Russo J, et al. The Pathways Study: a randomized trial of collaborative care in patients with diabetes and depression. Arch Gen Psychiatry 2004;61(10):1042-9.

47. Gilbody S, Bower P, Fletcher J, Richards D, Sutton AJ. Collaborative care for depression: a cumulative meta-analysis and review of longer-term outcomes. Arch Intern Med 2006;166(21):2314-21.

48. Wang PS, Simon G, Kessler RC. The economic burden of depression and the cost-effectiveness of treatment. Int J Methods Psychiatr Res 2003;12(1):22-33. 
49. Katon W, Unutzer J, Fan MY, Williams JW, Jr., Schoenbaum M, Lin EH, et al. Cost-effectiveness and net benefit of enhanced treatment of depression for older adults with diabetes and depression. Diabetes Care 2006;29(2):265-70.

50. Pinquart M, Duberstein PR, Lyness JM. Treatments for later-life depressive conditions: a meta-analytic comparison of pharmacotherapy and psychotherapy. Am. J. Psychiatry 2006;163(9):1493-501.

51. Barrett B, Byford S, Knapp M. Evidence of cost-effective treatments for depression: a systematic review. J Affect Disord 2005;84(1):1-13.

52. Timonen M, Liukkonen T. Management of depression in adults. Brit Med J 2008;336:435-439.

53. Bridges K, Goldberg D, Evans B, Sharpe T. Determinants of somatization in primary care. Psychol Med 1991;21(2):473-83.

54. Drayer RA, Mulsant BH, Lenze EJ, Rollman BL, Dew MA, Kelleher K, et al. Somatic symptoms of depression in elderly patients with medical comorbidities. Int J Geriatr Psychiatry 2005;20(10):973-82.

55. Gum AM, Arean PA, Bostrom A. Low-income depressed older adults with psychiatric comorbidity: secondary analyses of response to psychotherapy and case management. Int J Geriatr Psychiatry 2007;22(2):124-30.

56. Arean PA, Gum AM, Tang L, Unutzer J. Service use and outcomes among elderly persons with low incomes being treated for depression. Psychiatr Serv 2007;58(8):1057-64.

57. Katon WJ. Clinical and health services relationships between major depression, depressive symptoms, and general medical illness. Biol Psychiatry 2003;54(3):216-26. 
CHAPTER 8 


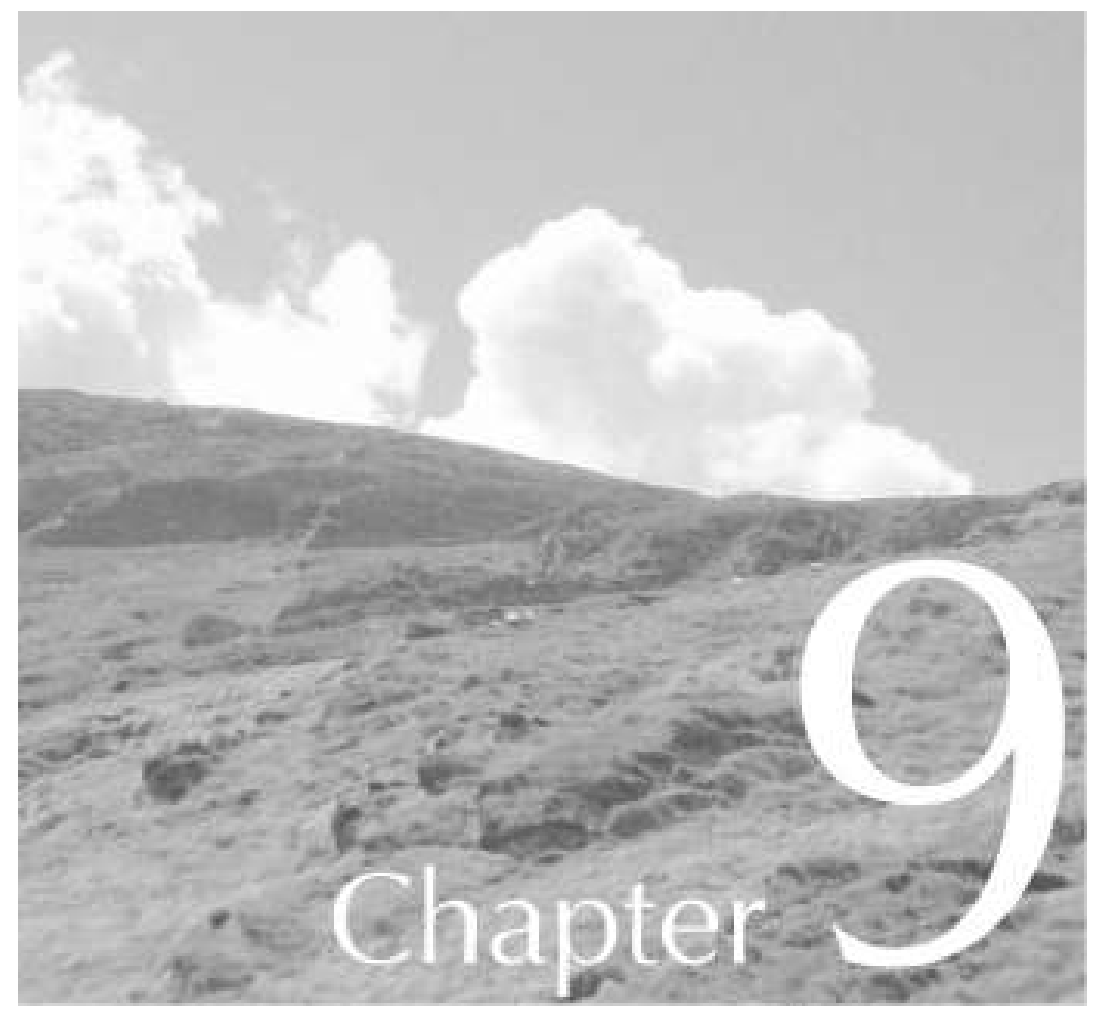

Summary

Samenvatting

Co-authors and affiliations

Dankwoord

About the author 
CHAPTER 9 


\section{Summary}

As the population ages, the health care system is facing an increasing number elderly persons with a chronic somatic disease, which in turn will increase the health care costs. Keeping patients in an optimal condition and preventing further disabilities are important goals in the treatment; however, co-occurring depression may push patients into a downward spiral and may accelerate the disablement process. Depression in chronically ill elderly often remains undetected and untreated in primary care. Major depression leads to lower quality of life, higher morbidity and mortality and is accompanied with higher health care costs and health care utilization. This also holds for minor depression, which is a sub-clinical form of major depression. Psychological treatments based in cognitive behavioural therapy (CBT) or self-management are preferable in chronically ill elderly persons over antidepressants. They provide lasting skills which may be of benefit in later stages of their disease and minimal formats may be acceptable in milder forms of depression as well, while the effectiveness of antidepressants in milder forms of depression has not been thoroughly established. The DELTA study (Depression in Elderly with Long-Term Afflictions) aimed to improve the detection and treatment of minor depression and mild to moderate major depression in chronically ill elderly persons. In a randomised controlled trial, a nurseled Minimal Psychological Intervention, based on CBT and self-management principles, was compared with care as usual. Aims of the study that were addressed in this thesis were 1) to evaluate both the effectiveness and cost-effectiveness of the nurseled Minimal Psychological Intervention (MPI) in reducing depressive symptoms and improving quality of life in elderly diabetic and chronic obstructive pulmonary disease (COPD) patients with a co-occurring minor to moderate depression, 2) to evaluate whether the effect of the MPI was generic across DM and COPD patients, 3) to evaluate the effect on COPD and diabetes-specific outcome measures, and 4) to test the reliability and validity of the Patient Health Questionnaire-9 (PHQ-9) that was used for depression screening.

Chapter 2 of this thesis describes the design of the DELTA study. Within 89 primary care practices in the south of the Netherlands, diabetic and COPD patients aged 60 year and over were screened for depression using a short depression screening questionnaire (Patient Health Questionnaire-9 - PHQ-9). Patients with a positive screening were invited to a diagnostic interview (Mini International Neuropsychiatric Interview - MINI) to confirm the depression diagnosis. Eligible patients were then invited to participate in the trial and were randomised. Data on the primary outcomes and on costs were further collected at one week, three, six (cost data only) and nine months after the intervention.

In Chapter 3, the reliability and validity of the PHQ-9 screening questionnaire were assessed. To assess reliability, a test-retest was conducted in 105 elderly diabetic and 
COPD patients. For the evaluation of the validity, data of 713 persons were available. Construct validity was assessed by calculating the correlation of PHQ-9 scores with scores on quality of life and disease severity measures. To assess criterion validity, sensitivity and specificity of the PHQ-9 were calculated for both the summed PHQ-9 score as well as the algorithm based PHQ-9 score, using the MINI diagnostic interview as the gold standard. Results from this study indicated that the PHQ-9 is a reliable instrument in elderly populations with diabetes or COPD. The construct validity of the PHQ-9 in this population was found acceptable. Further it was found that the algorithm based PHQ-9 score had high specificity but low sensitivity, indicating that it missed a larger percentage of depressed cases. The summed PHQ-9 score however, had both high sensitivity and specificity, and should be preferred over the algorithm based PHQ-9 score in screening elderly diabetic and COPD patients.

Chapter 4 presents the effects of the intervention on depressive symptoms and quality of life and provides an answer to the question whether or not the effects of the MPI are generic across diseases. In total, 361 patients with diabetes type II and/or COPD were included in the study and were randomly allocated to the nurse-led MPI or usual care. At baseline, groups were comparable in terms of demographic and socio-economic factors and on the outcomes depressive symptoms (Beck Depression Inventory) and quality of life (Short Form-36). During the trial, 33\% of the patients dropped out but drop-out was non-differential between groups. The MPI had a positive effect on depressive symptoms, with increasing differences over time and a significant difference at nine months after the intervention. Patients receiving the intervention were also three times more likely to show a reduction of $50 \%$ or more of baseline depressive symptoms. The effect on depressive symptoms was found to be generic across diseases. The intervention further had an effect on quality of life, although this was only observed in the diabetic patients.

Chapter 5 describes the effect of the MPI on diabetes-specific outcomes. Of the total DELTA-population, 208 patients had diabetes type II (185 DM only; 23 also with COPD) and were included in the analysis. Outcome measures used were diabetes symptom distress (Diabetes Symptoms Checklist-Revised), emotional distress (Problem Areas in Diabetes) and glycemic control. For glycemic control, $\mathrm{HbA1c}$ data were collected from primary care and hospital records, and were available for 135 persons. As results of analyses on symptom and emotional distress showed only limited effects, sub-group analyses indicated that the intervention had a significant effect on both symptom and emotional distress in higher-educated with medium to large effect sizes, but not in lower-educated patients. Further, we found a significant effect of the intervention on symptom distress in men, and on emotional distress in patients with a diabetes duration $<7$ years. The intervention further had an effect on glycemic control. A significant difference in trend over time was observed, with a significantly lower mean HbA1c level in the intervention group compared with the control groups at nine months after the intervention (between-group HbA1c difference 0.5\%). As these 
results indicate that not all groups of patients benefited equally from the intervention, further exploration is necessary.

Chapter 6 reports on the effects of the intervention in COPD patients. Outcomes measures used were COPD-specific quality of life (Saint George's Respiratory Questionnaire), depressive symptoms (Beck Depression Inventory) and symptoms of anxiety (Symptom Checklist). Anxiety was included because COPD patients often experience anxiety and we expected the intervention to influence symptoms of anxiety as well. In total, 187 COPD patients (176 COPD only; 11 also with diabetes) were included in the analyses. The intervention group on average had significantly less symptoms of depression and anxiety at nine months after the intervention than the control group. Although in earlier analyses no effects were found on generic quality of life in COPD patients (Chapter 5), these analyses showed that the intervention did have significant effects on COPD-specific quality of life, with medium-sized effects.

In Chapter 7, the results from the economic evaluation are presented. Such information is of great value to policy makers and health insurers. To evaluate costeffectiveness and cost-utility, data on all relevant costs were collected, including program costs, health care costs, patient and family costs, and productivity losses. For the effect estimate, quality-adjusted life years based on the EuroQol-5 and on depression-free days were calculated. Two-hundred and twenty-eight persons were included in the analyses. Results indicated that between-group differences in effect and in costs were in favour of the intervention, although differences were not significant. Bootstrap analyses of the incremental cost-utility ratio revealed that the intervention was dominant, with a $63 \%$ probability that the intervention is both more effective and less costly than usual care.

Finally, in Chapter 8, the main results are put into a broader perspective. First a summery of the main results is given and answers to the research questions are provided. Overall, the nurse-led Minimal Psychological Intervention was found both effective and cost-effective and the effect on depressive symptoms was generic across diabetes and COPD. The summed PHQ-9 score was found to be a reliable and valid tool for screening elderly diabetic and COPD patients for depression. Dissemination of the intervention in regular care or existing disease-management programs seems the logical next step, although certain issues around target population, monitoring and training of nurses need to be addressed. Topics for the future research agenda include the assessment of the long-term effects of depression treatment on the course of chronic illnesses and the interaction between depression and chronic somatic disease. 
CHAPTER 9 


\section{Samenvatting}

Door de vergrijzing van de populatie, zal er een toename komen van het aantal ouderen met een chronische ziekte en zullen tevens de kosten voor de gezondheidszorg stijgen. Om verdere invaliditeit van patiënten te voorkomen, is het daarom van belang om chronisch zieke ouderen in een zo optimaal mogelijke gezondheidstoestand te houden. Een depressie kan er echter voor zorgen dat mensen in een neerwaartse spiraal terecht komen en dit kan het invaliditeitsproces versnellen. In de eerstelijnszorg wordt bij chronisch zieke ouderen depressie vaak niet herkend of behandeld. Depressie leidt tot een slechtere kwaliteit van leven, hogere morbiditeit en mortaliteit en gaat gepaard met hogere gezondheidszorgkosten en zorggebruik. Hetzelfde geldt voor de lichte, subklinische vorm van depressie. De behandeling van depressie met psychologische interventies, zoals cognitieve gedragstherapie (CBT) en zelfmanagement, zijn te prefereren boven behandeling met antidepressiva, omdat mensen door psychologische behandelingen vaardigheden ontwikkelen die van blijvend nut kunnen zijn. Ook zijn psychologische behandelingen voor mensen met mildere vormen van depressie acceptabeler dan antidepressiva, waarvan de werking in mildere vormen van depressie nog niet goed is vastgesteld. De DELTA studie (Depression in Elderly with Long-Term Afflictions) beoogde de herkenning en behandeling van depressie bij chronisch zieke ouderen te verbeteren. In een gerandomiseerde studie werd een Minimale Psychologische Interventie (MPI), die gebaseerd was op CBT en zelfmanagement principes en werd gegeven door een verpleegkundige, vergeleken met gebruikelijke zorg. Doelen van de studie die zijn behandeld in dit proefschrift waren (1) het evalueren van de effectiviteit en kosteneffectiviteit van de MPI in het verminderen van depressieve symptomen en het verbeteren van de kwaliteit van leven van ouderen met diabetes type II of chronisch obstructieve pulmonaire aandoeningen (COPD) die tevens lichte tot matige depressie hadden, (2) het evalueren of het effect van de MPI generiek is over diabetes en COPD patiënten, (3) het evalueren van de effecten van de MPI op COPD- en diabetesspecifieke uitkomstmaten en 4) het evalueren van de betrouwbaarheid en validiteit van de Patient Health Questionnaire-9 (PHQ-9) die in de DELTA studie werd gebruikt om mensen met depressie op te sporen.

Hoofdstuk 2 van dit proefschrift beschrijft de opzet van de DELTA studie. Alle diabetes en COPD patiënten van 60 jaar en ouder uit 89 huisartspraktijken in ZuidLimburg werden gescreend op depressie door middel van een korte screeningsvragenlijst (PHQ-9). Patiënten met een positieve screening werden uitgenodigd voor een diagnostisch interview (Mini International Neuropsychiatric Interview - MINI) om de diagnose depressie te bevestigen. Geschikte patiënten werden daarna uitgenodigd deel te nemen aan het onderzoek. Dataverzameling door middel van schriftelijke vragenlijsten en kostendagboekjes vond plaats bij instroom in het onderzoek, één week na de behandeling, en drie, zes en negen maanden na behandeling. 
Hoofdstuk 3 beschrijft de betrouwbaarheid en validiteit van de Patient Health Questionnaire-9 (PHQ-9) in een oudere populatie diabetes en COPD patiënten. Om de betrouwbaarheid van deze korte screeningsvragenlijst te bepalen werd een testhertest uitgevoerd onder 105 oudere diabetes en/of COPD patiënten. Voor het bepalen van de validiteit werd gebruik gemakkt van gegevens van 713 ouderen met diabetes en/of COPD. Construct validiteit werd bepaald door het berekenen van correlaties tussen de PHQ-9 en scores op uitkomstmaten als kwaliteit van leven en ziekte-ernst. Om criterium validiteit vast te stellen werd de sensitiviteit en specificiteit van de PHQ-9 berekend, voor zowel de somscore als de algoritme-gebaseerde score van de PHQ-9, waarbij de MINI als gouden standaard werd gebruikt. Uit de resultaten bleek dat de PHQ-9 een betrouwbaar screeningsinstrument voor depressie is bij ouderen met diabetes of COPD. De construct validiteit van de PHQ-9 was acceptabel. Betreffende de criterium validiteit werd gevonden dat de het algoritme-gebaseerde PHQ-9 score een hoge specificiteit had, maar een lage sensitiviteit, wat betekent dat er veel mensen met een depressie onopgemerkt bleven. De somscore van de PHQ-9 had zowel een hoge sensitiviteit als en een hoge specificiteit en zou daarom geprefereerd moeten worden boven de algoritme-gebaseerde score bij gebruik van de PHQ9 bij ouderen met diabetes of COPD.

In Hoofdstuk 4 worden de resultaten van de effecten van de interventie op depressieve symptomen en kwaliteit van leven gepresenteerd en wordt tevens een antwoord gegeven op de vraag of de effecten generiek zijn over de ziektes. In totaal werden 361 patiënten met diabetes type II of COPD in het onderzoek ingesloten en willekeurig toegewezen aan de interventiegroep die de Minimale Psychologische Interventie (MPI) van de verpleegkundige ontving of aan de controlegroep die gebruikelijke zorg kreeg. Bij aanvang van de studie waren beide groepen goed vergelijkbaar wat betreft demografische en sociaaleconomische factoren en vergelijkbaar wat betreft scores op de uitkomstmaten depressieve symptomen (Beck Depression Inventory) en kwaliteit van leven (SF-36). Gedurende het onderzoek viel $33 \%$ van de patiënten uit, maar de uitval was niet verschillend tussen de interventie en controlegroep. De MPI bleek een positief effect te hebben op depressieve symptomen, waarbij het verschil in depressieve symptomen tussen beide groepen groter werd met de tijd en op negen maanden na de interventie statistisch significant was. Ook hadden patiënten in de interventiegroep een driemaal hogere kans om een vermindering van depressieve symptomen van $50 \%$ of meer door te maken dan patiënten in de controlegroep. Het effect was tevens generiek over beide chronische ziektes. De interventie had tevens een effect op kwaliteit van leven, alhoewel dit alleen in diabetes patiënten werd waargenomen.

Hoofdstuk 5 omschrijft de effecten van de interventie op diabetes-specifieke uitkomstmaten. Van de totale DELTA-populatie hadden 208 patiënten diabetes type II (185 alleen DM; 23 naast DM ook COPD), en deze 208 patiënten werden gebruikt in 
de analyses. De uitkomstmaten waren (hinder van) specifieke diabetes klachten (Diabetes Symptom Checklist-Revised), diabetes specifieke emotionele problemen (Problem Areas In Diabetes) en glycemische controle. Voor analyses op glycemische controle werden HbA1c waarden van deelnemers opgevraagd in huisartspraktijken en het ziekenhuis indien beschikbaar; dit was voor 135 patiënten het geval. Omdat analyses in de totale diabetes-populatie slechts een beperkt effect van de interventie liet zien, werden subgroepanalyses uitgevoerd. Uit deze subgroepanalyses bleek dat de interventie een significant effect had op (hinder van) diabetes klachten en emotionele problemen in hoger opgeleide patiënten met matige tot grote effecten, maar niet in lager opgeleide diabetes patiënten. Tevens werd gevonden dat de interventie bij mannen een significant effect had op (hinder van) diabetes klachten, en bij patiënten met minder dan 7 jaar diabetes een significant effect had op emotionele problemen. De interventie bleek ook een positief effect te hebben op glycemische controle. Er werd een significant verschil gevonden in de trend van Hba1c over tijd, met een significant lagere Hba1c waarde in de interventiegroep na 9 maanden (verschil HbA1c groepen 0.5\%). Omdat de resultaten van deze studie indiceren dat niet iedereen evengoed profiteert van de interventie, is verdere exploratie naar werkzaamheid in verschillende groepen diabetes patiënten noodzakelijk.

Hoofdstuk 6 beschrijft de effecten van de interventie op COPD patiënten. Als uitkomstmaten werden gebruikt: COPD-specifieke kwaliteit van leven (Saint George's Respiratory Questionnaire), depressieve symptomen (Beck Depression Inventory) en symptomen van angst (Symptom Checklist). Angst werd meegenomen omdat COPD patiënten vaak gevoelens van angst ervaren en omdat verwacht werd dat de interventie ook op angst invloed zou hebben. In totaal werden 187 COPD patiënten ingesloten in de analyse (176 alleen COPD; 11 naast COPD ook diabetes). Uit de analyses bleek dat patiënten in de interventiegroep gemiddeld minder symptomen van depressie en angst hadden dan patiënten uit de controlegroep. Dit verschil was significant op 9 maanden na de behandeling. Alhoewel er in eerdere analyses geen effecten van de interventie werden gevonden op generieke kwaliteit van leven in COPD patiënten (Hoofdstuk 5), werden in de analyses van COPD-specifieke kwaliteit van leven wel significante effecten gevonden, met gemiddelde effect groottes.

In Hoofdstuk 7 worden de resultaten van de economische evaluatie beschreven. Informatie over kosteneffectiviteit van een behandeling is belangrijk voor beleidsmakers en verzekeraars. Voor het evalueren van kosteneffectiviteit en kostenutiliteit werden gegevens over alle relevante kosten verzameld, inclusief programma kosten, gezondheidszorgkosten, patiënt en familiekosten en productiviteitsverlies. Voor de effect schatting werden quality-adjusted life years berekend op basis van de EuroQol5 en op depressie-vrije dagen. Er werden 228 personen in de analyses ingesloten. Analyses lieten verschillen zien in kosten en effecten tussen interventiegroep en controlegroep in het voordeel van de interventiegroep, maar de verschillen waren niet significant. Bootstrap analyses van de incrementele kostenutiliteits-ratio wezen 
uit dat de interventie dominant was met een $63 \%$ waarschijnlijkheid dat de interventie effectiever is dan gebruikelijke zorg tegen lagere kosten.

Tot slot worden in Hoofdstuk 8 de resultaten in een breder perspectief geplaatst. Eerst wordt een samenvatting van de gevonden effecten gegeven die een antwoord geven op de onderzoeksvragen. De Minimale Psychologische Interventie, uitgevoerd door een verpleegkundige, bleek zowel effectief als kosteneffectief te zijn. Het effect van de interventie op depressieve symptomen was generiek over beide chronische ziekten (DM en COPD). De PHQ-9 somscore bleek een betrouwbare en valide methode te zijn om te screenen op depressie in ouderen met diabetes of COPD. Disseminatie van de interventie in de gebruikelijke zorg of in bestaande diseasemanagement programma's lijkt de volgende logische stap. Er dienen echter eerst een aantal zaken rondom de doel-populatie, monitoring en opleiding van verpleegkundigen te worden aangepakt. Toekomstig onderzoek zou zich onder andere moeten richten op het evalueren van effecten van depressiebehandeling op langere termijn en de invloed op het beloop van de onderliggende chronische aandoening, evenals de interactie tussen chronische ziekten en depressie. 


\section{Co-authors and affiliations}

$\mathrm{H}$ (Hans) Bosma, PhD

Maastricht University, Faculty of Health, Medicine and Life Sciences, Department of Social Medicine, School for Public Health and Primary Care (CAPHRI), Maastricht, the Netherlands

$\mathrm{NH}$ (Niels) Chavannes, MD, PhD

Health Centre Zuiderkroon, Rotterdam, the Netherlands

Maastricht University, Faculty of Health, Medicine and Life Sciences, Department of General Practice, School for Public Health and Primary Care (CAPHRI), Maastricht, the Netherlands

JPM (Jos) Diederiks, PhD

Maastricht University, Faculty of Health, Medicine and Life Sciences, Maastricht, the Netherlands

JThM (Jacques) van Eijk, PhD

Maastricht University, Faculty of Health, Medicine and Life Sciences, Department of Social Medicine, School for Public Health and Primary Care (CAPHRI), Maastricht, the Netherlands

SMAA (Silvia) Evers, PhD

Maastricht University, Faculty of Health, Medicine and Life Sciences, Department of Department of Health Organization, Policy and Economics, School for Public Health and Primary Care (CAPHRI), Maastricht, the Netherlands

CCM (Karianne) Jonkers, MSc

Maastricht University, Faculty of Health, Medicine and Life Sciences, Department of Social Medicine, School for Public Health and Primary Care (CAPHRI), Maastricht, the Netherlands

GIJM (Ruud) Kempen, PhD

Maastricht University, Faculty of Health, Medicine and Life Sciences, School for Public Health and Primary Care (CAPHRI), Maastricht, the Netherlands

JA (André) Knottnerus, MD, PhD

Maastricht University, Faculty of Health, Medicine and Life Sciences, Department of General Practice, School for Public Health and Primary Care (CAPHRI), Maastricht, the Netherlands 


\section{CHAPTER 9}

JAMJ (Jaap) Meijer, MD

Virenze Group Practice for Psychotherapy and Occupational Counselling, Rijckholt, the Netherlands

JF (Job) Metsemakers, MD, PhD

Maastricht University, Faculty of Health, Medicine and Life Sciences, Department of General Practice, School for Public Health and Primary Care (CAPHRI), Maastricht, the Netherlands

BWJH (Brenda) Penninx, PhD

VU University Medical Center, Department of Psychiatry and EMGO Institute, Amsterdam, the Netherlands 


\section{Dankwoord}

Als na 5 jaar werken de eindstreep dan eindelijk in zicht is en het eindresultaat te bewonderen is in de vorm van dit proefschrift, dan is het ook de hoogste tijd om alle mensen te bedanken die aan de totstandkoming van dit boekje hebben bijgedragen. Jacques, dat de DELTA-studie je na aan het hart ligt, zal niemand in de projectgroep ontgaan zijn. Mede dankzij jouw grote inzet en inspanningen om huisartsen te werven - wat niet altijd zonder slag of stoot ging - wisten we de beoogde patiëntenaantallen te bereiken. Het was plezierig om met je samen te werken; ik kon altijd binnen komen lopen en je gaf me de vrijheid en ruimte om de dingen op mijn eigen manier aan te kunnen pakken. Heel erg bedankt! Hans, dank je wel voor je uitstekende begeleiding in de afgelopen jaren. Het was fijn om met iemand met zoveel statistische kennis te kunnen samenwerken; ik heb er veel van geleerd. Met plezier kijk ik terug op onze statistiek discussies die me altijd weer nieuwe energie gaven om ermee verder te gaan. André, al kwam jij er als promotor pas in een later stadium bij, je was meteen een volwaardig lid van het promotoren-team, hetzij op wat grotere afstand. Met je commentaar op mijn artikelen wist je altijd feilloos de zere plekken aan te wijzen. Bedankt voor de prettige samenwerking.

Daarnaast wil ik de leden van de begeleidingscommissie en/of co-auteurs noemen: Ruud Kempen, Jos Diederiks, Job Metsemakers, Brenda Penninx, Silvia Evers, Jaap Meijer, Niels Chavannes en Bert Vrijhoef. Hartelijk dank voor jullie bijdragen in de afgelopen jaren. Het was prettig en leerzaam om met enige regelmaat input te krijgen vanuit een andere invalshoek. Bedankt dat ik van jullie expertise gebruik mocht maken. Ook wil ik iedereen bedanken die betrokken is geweest bij het opzetten en uitvoeren van het trainingsprogramma voor de verpleegkundigen: Klaas van der Meer, Wiljo Brenninkmeijer, Adriaan Honig en Carsten Leue.

Als er vier mensen zijn wiens werk van cruciaal belang is geweest voor het DELTA onderzoek, dan zijn dat wel de DELTA-verpleegkundigen: Kitty Daemen, Francine Hendriks, Henny Geelen en Mieke Witte. Dames, jullie hebben geweldig werk verricht en het was dan ook een voorrecht om met jullie te mogen samenwerken! Ik kijk met veel plezier terug op de tijd van het veldwerk: aan de mooie verhalen die jullie vertelden over jullie ervaringen met de patiënten, de beschrijvingen van het mooie landschap van Zuid-Limburg ("Waar ik nu toch weer sta: Het is hier zo mooi! Jammer dat ik geen camera op mijn telefoon heb!") en aan de gezelligheid. Jullie vormden een geweldig team en ik ben dan ook supertrots op jullie!

Tijdens het onderzoek hebben verschillende mensen meegeholpen in het voorbereiden, opzetten en uitvoeren van de dataverzameling. Wendy, je hebt bergen werk verzet in de deelnemende huisartspraktijken en daar was soms flink wat doorzettingsvermogen voor nodig, bedankt! Dorien, bedankt voor je ondersteuning in de 
data-invoer en logistieke taken waarmee je ons veel werk uit handen hebt genomen. Dan de mensen van MEMIC: Anita en Alfons, bedankt voor het uitdenken, ontwerpen en bouwen van het logistiek systeem. Het kostte wat tijd en flink wat inspanning, maar dan heb je ook wat! Marlène en alle enquêtrices, bedankt voor jullie aandeel in de data-verzameling. Het was geen gemakkelijke klus maar jullie hebben het tot een prima einde volbracht. Ook wil ik graag alle deelnemende huisartspraktijken en deelnemers aan de studie bedanken voor hun medewerking aan het onderzoek.

Het grootste deel van mijn promotie-traject heb ik doorgebracht bij de sectie Medische Sociologie. Graag wil ik alle oud-collega's bedanken voor de gezelligheid in de dagelijkse theepauzes en gezamelijke lunches en voor de getoonde interesse in de DELTA-studie. De laatste periode van mijn promotie-traject bracht ik door op de vakgroep Sociale Geneeskunde. Alhoewel ik er maar kort werkzaam ben geweest, wil ik iedereen bedanken voor de korte, maar leuke tijd.

Bijzonder dank ook aan alle mede-AIO's. Ik kijk met plezier terug op de kritische AIO-soep-avondjes en de gezellige uitstapjes die we samen hebben gemaakt. ledereen bedankt voor de leuke en leerzame tijd! Rixt en Gonnie, ik ga onze bijkletsmomenten missen! Karianne, toen we onze samenwerking op het DELTA-onderzoek begonnen, was het al snel duidelijk dat de selectiecommissie die ons had uitgekozen, goed werk had geleverd. Het was af en toe gewoon bizar om te ontdekken hoeveel overeenkomsten we hadden (Tja, die gordijntjes van de Alpenkreuzer, hè!). Mede door al die overeenkomsten in denken en doen is onze samenwerking tot een succes geworden. Bedankt voor de gezelligheid, de fijne samenwerking en je steun door de jaren heen. Ik kan me geen betere paranimf voorstellen!

Verder wil ik vrienden, familie en kennissen bedanken voor hun getoonde interesse in mijn werk. Olga, bedankt voor de gezellige mail-correspondentie van de afgelopen jaren! Mayke, dat mijn 'big Sizz' mijn paranimf zou worden, stond voor mij al lang vast. Bedankt voor je interesse, steun en gezelligheid door alle jaren heen. Dat onze shop-weekendjes en vakanties ook in de toekomst een vervolg mogen krijgen, ook al is de afstand tussen ons straks wat groter! Pap en mam, met jullie opvoeding, downto-earth instelling, liefde en onvoorwaardelijke steun, hebben jullie me een geweldige basis meegegeven. Het heeft me gemaakt tot wie ik ben. Bedankt!

Femke 


\section{About the author}

Femke Lamers was born on May 1, 1980 in Warnsveld, the Netherlands. After completing her secondary education (VWO) at the Baudartius College in Zutphen, she started her study Nutrition and Health at the Wageningen University in August 1998. She completed the specialization Epidemiology at the Faculty of Human Nutrition and Epidemiology at the Wageningen University, and the specialization Lifestyle and Public Health at the Municipal Health Services in Utrecht. She concluded her study with an internship at the Centre for Chronic Disease Epidemiology at the National Institute for Public Health and the Environment (RIVM), in Bilthoven. She obtained her Masters degree in January 2003 and was registered as an epidemiologist with the Netherlands Epidemiological Society in September of that year.

In July 2003 the author started as a PhD student at the Faculty of Health Medicine and Life Sciences of the Maastricht University and School for Public Health and Primary Care (CAPHRI). There, she worked on the DELTA study, a randomised controlled trial that evaluated the effectiveness and cost-effectiveness of a nurse-led minimal psychological intervention on reducing depressive symptoms and improving quality of life in chronically ill elderly persons. Since May 2008 she works as a postdoctoral research fellow on the Netherlands Study of Depression and Anxiety (NESDA) at the VU Medical Center/GGZ BuitenAmstel in Amsterdam. 